\title{
Intermittent Control as a Model of Mouse Movements
}

\author{
J. ALBERTO ÁLVAREZ MARTÍN and HENRIK GOLLEE, James Watt School of Engineering, \\ University of Glasgow \\ JÖRG MÜLLER, Institute for Computer Science, University of Bayreuth \\ RODERICK MURRAY-SMITH, School of Computing Science, University of Glasgow
}

\begin{abstract}
We present Intermittent Control (IC) models as a candidate framework for modelling human input movements in Human-Computer Interaction (HCI). IC differs from continuous control in that users are not assumed to use feedback to adjust their movements continuously, but only when the difference between the observed pointer position and predicted pointer positions becomes large. We use a parameter optimisation approach to identify the parameters of an intermittent controller from experimental data, where users performed onedimensional mouse movements in a reciprocal pointing task. Compared to previous published work with continuous control models, based on the Kullback-Leibler divergence from the experimental observations, IC is better able to generatively reproduce the distinctive dynamical features and variability of the pointing task across participants and over repeated tasks. IC is compatible with current physiological and psychological theory and provides insight into the source of variability in HCI tasks.
\end{abstract}

\section{CCS Concepts: • Human-centered computing $\rightarrow$ HCI theory, concepts and models;}

Additional Key Words and Phrases: Control theory, modelling, pointing, intermittent control

\section{ACM Reference format:}

J. Alberto Álvarez Martín, Henrik Gollee, Jörg Müller, and Roderick Murray-Smith. 2021. Intermittent Control as a Model of Mouse Movements. ACM Trans. Comput.-Hum. Interact. 28, 5, Article 35 (August 2021), 46 pages. https://doi.org/10.1145/3461836

\section{INTRODUCTION}

One of the most important areas of study of Human-Computer Interaction (HCI) is how humans can interact with computers to input information into computers or control their state. Most applied human-computer input is via activity of the user's neuromuscular system (NMS) causing movement of their body, which is sensed by input devices and produces a change in the state of the computer. The most widely studied example of this in $\mathrm{HCI}$ is mouse movement, where a user's hand moves a mouse to change the cursor position. The goal of this article is to provide a physiologically plausible model of such user movements and the associated movement variability. While our long-term objective is to understand movement during interaction with computers

J. A. Á. M., H. G., and R. M.-S. acknowledge funding support from EPSRC grant EP/R018634/1, Closed-loop Data Science. Authors' addresses: J. A. Álvarez Martín and H. Gollee, James Watt School of Engineering, University of Glasgow, Glasgow G12 8QQ, UK; emails: Alberto.Alvarez-Martin@glasgow.ac.uk, Henrik.Gollee@glasgow.ac.uk; J. Müller, Institute for Computer Science, University of Bayreuth, Bayreuth 95447, Germany; email: Joerg.Mueller@uni-bayreuth.de; R. Murray-Smith, School of Computing Science, University of Glasgow, Glasgow G12 8RZ, UK; email: Roderick.MurraySmith@glasgow.ac.uk.

Permission to make digital or hard copies of all or part of this work for personal or classroom use is granted without fee provided that copies are not made or distributed for profit or commercial advantage and that copies bear this notice and the full citation on the first page. Copyrights for components of this work owned by others than the author(s) must be honored. Abstracting with credit is permitted. To copy otherwise, or republish, to post on servers or to redistribute to lists, requires prior specific permission and/or a fee. Request permissions from permissions@acm.org.

(C) 2021 Copyright held by the owner/author(s). Publication rights licensed to ACM.

1073-0516/2021/08-ART35 \$15.00

https://doi.org/10.1145/3461836 
in general, in this article, we focus on a simple core task: aimed movements of a mouse cursor towards a static target of a certain width at a certain distance.

Movement in interaction with computers is inherently dynamic and clearly happens in a feedback loop. Users observe the current state of the computer (e.g., cursor position) and adjust their movements to change this state into the state they desire-"movements only make sense when they are precisely located in time and space that is, when they are part of an action, seeking to accomplish a goal. In this sense, simple goal-directed movements, such as pointing and grasping, can be considered to be the building blocks of more complex actions" [Bootsma et al. 2004]. Control theory is the mathematical framework for systems with feedback which are achieving specific objectives, and its relevance for HCI is reviewed in Murray-Smith [2018] and Müller et al. [2017].

In this article, we explore the benefits of interpreting interaction with computers as intermittent control (IC). In IC, open-loop control trajectories are generated based on an internal estimate of the state of interactions using a predictive model of how this state will evolve over time. The open-loop trajectories are intermittently updated with feedback information from continuous observations of the systems. Only if the observed state deviates from the predicted state, do they update their prediction and control accordingly. Loram et al. [2014] explain why the IC perspective is more physiologically plausible as an explanation of human motor control than our previous continuous control (CC) perspectives of interaction with computers (e.g., Müller et al. [2017]). It has been shown that humans are generally not able to adjust their movement continuously [Navas and Stark 1968]. The switching and intermittency inherent in IC provides a powerful explanation of the source of variability in human movement, and potentially, it can also explain the emergence of phenomena that are difficult to explain from a CC perspective, such as submovements.

\subsection{Beyond Fitts' Law}

Since the advent of graphical user interfaces, aimed movements towards a spatially defined target have become a primary means of input to computers. It is well known that all movements involve variability in their performance. For aimed movements, this has been described in the "speed-accuracy tradeoff", modelled through Fitts' law [Fitts 1954]. Fitts' law allows the prediction of movement time $M T$ as a function of distance $D$ to and width $W$ of the target, as $M T=a+b \log (D / W+1)$ in the Shannon formulation [MacKenzie 1992]. The application of Fitts' law to understand interaction with computers is often viewed as one of the greatest successes of the field of HCI [Guiard and Beaudouin-Lafon 2004a]. Fitts' law allows for the model-based evaluation of user interfaces, reducing the number of necessary user tests, and serves in the automatic optimisation of user interfaces such as Graphical User Interface (GUI) layouts or keyboards. Perhaps most importantly, it has sharpened the intuition of generations of user interface designers about the role of the users' motor control processes in interaction with computers.

One of the strengths, but also limitations, of Fitts' law is that it reduces the complexity of human movement to a single number, the movement time, but it makes no testable predictions about the process of the movement. The Fitts' law perspective offers no explanation of the causal relationship that links conditions and outcomes in pointing. Neither the biomechanical, perceptual, and cognitive properties of the human nor the properties of the computer interface such as input sensors, delays, jitter, or style of feedback can be understood from this perspective. It does not allow any understanding of why a certain user interface, interaction technique, or input device is better than another. In particular, the trajectory of the pointer, velocities, and accelerations cannot be explained by Fitts' law, so a richer understanding of the broader process of movement is lost, and as Bootsma et al. [2004] observe, "while the duration of movement constitutes a particularly pertinent global measure of behavior, useful in many different contexts, it does not allow a full appreciation of the processes underlying behavioral organization. In the domain of perceptuomotor 
control, it is widely accepted that a more fine-grained window into these processes is available through the kinematics of movement". Perhaps most critically, Fitts' law is limited to aimed movements. This may make it more difficult for the field of HCI to think beyond aimed movements and point-and-click as the foundation of interface design. In order to develop a deeper understanding in HCI of how users create input to computers by moving their bodies, we propose that we need to take a control theoretic perspective, and we are exploring models which are appropriate for the representation of purposeful human movement.

\subsection{Paper Structure}

In this article, we present a model of aimed mouse movement in the interaction with computers, based on IC. We build on an existing dataset to fit the parameters of our event-driven intermittent controller, allowing a comparison with the continuous implementations proposed in Müller et al. [2017]. The objectives of this article are as follows:

(1) identify the parameters of an intermittent controller from the experimental mouse movement data by using an optimisation approach;

(2) examine the ability of IC to generatively reproduce and explain distinctive dynamical features of the pointing task such as the velocity profile and the variability observed across participants;

(3) introduce IC as a plausible framework to understand and model user movement in the interaction with computers, as a novel analytic and practical tool for HCI research and practice.

The content of this article is organised as follows: After reviewing related work in Section 2, a general introduction to the IC framework is given in Section 3, focusing on its role in pointing and human variability. We highlight that the model is based on human physiological insight, and includes predictive properties which are key to understanding and modelling human behaviour in interaction contexts. Section 4 then provides a more mathematically formal description of the theoretical details of IC. Sections 5-7 provide descriptions of the experimental dataset and the models and optimisation process used for the analysis. Sections 8-9 provide the modelling results, demonstrating that IC models provide improved modelling of the variability and dynamics of mouse movements compared to previous publications. In Sections 11 and 12, we discuss the conceptual and practical advantages of the model, and give an outlook on further developments.

\section{RELATED WORK}

\subsection{Fitts' Law and Information Theory}

Since the original work [Fitts 1954], Fitts' relationship has been used in a wide range of HCIrelevant publications. For more background, Guiard and Beaudouin-Lafon [2004b] introduce a special issue celebrating 50 years of Fitt's law, and Soukoreff and MacKenzie [2004] review 27 years of Fitts' law in HCI and make recommendations to HCI researchers wishing to construct Fitts' law models for movement time prediction, or for the comparison of conditions in an experiment. Wobbrock et al. [2008] show that Fitts' law implies a predictive error rate model, and that the effect on error rate of target size $W$ is greater than that of target distance $D$.

Dynamic aspects of Fitts' law tasks are explored in Billon et al. [2000], Bootsma et al. [2004], and Guiard [1993], and Jagacinski [1977] and Jagacinski and Flach [2003] link Fitts' law models to first- and second-order control models. While most Fitts' law experiments are artificial lab studies, Chapuis et al. [2007] collected kinematic data from 24 users over several months as part of their normal interactions with a computer. Some papers have explored dynamic relationships in implementations of motor and display spaces, while not taking an explicitly control-theoretic 
perspective. While pointing in the physical world is constrained by physical laws, Balakrishnan [2004] observed that pointing in the virtual world does not have to abide by the same constraints, and compared approaches aiming to "beating" Fitts' law by artificially reducing the target distance, increasing the target width, or both, essentially adapting the dynamics of the control task. Blanch et al. [2004] introduced the concept of Semantic pointing, which manipulates control-display gain, using two independent sizes for targets in motor space, adapted to its importance for the manipulation, and in visual space, adapted to the amount of information it conveys. Their experimental results suggested that the performance of semantic pointing is given by Fitts' index of difficulty (ID) in motor rather than visual space.

\subsection{Study of Movement from an Intermittent Perspective}

Control of body movements has been modelled as both discrete and continuous processes, and there are strong arguments for either perspective:

In favour of the continuous perspective, the human hand is a physical inertial system, which changes its position smoothly over time. Although difficult aimed movements can be segmented into submovements, identifying the beginning and end of submovements is notoriously difficult. In some movements, there do not seem to be submovements at all. The continuous perspective can also explain other movements which are not aimed, such as following a moving target, steering a cursor along some path, controlling a character or vehicle in a video game, and so on. Understanding movement as a series of discrete submovements does not appear to concisely capture these phenomena.

In favour of the discrete perspective, data show that humans are not capable of continuously controlling their movements in the same way as an engineered CC system. Craik first reported this in Craik [1947, 1948]. In particular, the psychological refractory period [Telford 1931] dictates that humans cannot react to further changes in the environment for a certain time after they reacted to a change: The reaction onset to a second stimulus which follows shortly after the first is delayed as the human is refractory during this time. Crucially, a control paradigm based on continuous feedback is unable to explain refractoriness in human motor control.

Interestingly, the perspectives of discrete movements vs CC may be less contradictory than it first seems. Craik proposed that a series of discrete movements could appear to be continuous in nature. From this perspective, the human hand moves continuously, but the way the hand moves is only changed at certain points in time, or intermittently. Craik's manual tracking experiments led to the conclusion that the human operator uses an intermittent approach, dictated by refractoriness, when tracking discrete reference steps, and that manual control can be seen as the execution of discrete actions that are computed based on the available sensory information and applied as individual open-loop trajectories that are not modified by feedback until completed. Since then, intermittency from the perspective of human motor control has been studied extensively by the physiology community [Loram et al. 2006; Loram and Lakie 2002; Navas and Stark 1968; Nielson 1999; Oytam et al. 2005], and as a consequence, computational frameworks have been developed to model the human operator as an intermittent controller using concepts of modern control theory. On the engineering side, the intermittent controller has its origins in the implementation of model-based predictive control (MPC) in the presence of hard constraints [Ronco et al. 1999] and the observer-predictor-feedback architecture from Kleinman [1969] and Kleinman et al. [1970]. It is important to note that, as explained above, intermittency can masquerade as CC, in particular when controlling an inertial system, and when the controller has a good internal model of the system [Gawthrop et al. 2011].

Schmidt's law [Schmidt et al. 1978] addresses variability and dynamics by manipulating amplitude and movement time and measuring the effective target width $W_{e}$, leading to the relation 
$W_{e}=k \frac{W}{M T}$. The insight behind Schmidt's law is that the human controls movement via discrete force impulses and the overall variability is from the variation of the magnitude and duration of the applied force. Meyer et al. [1988, 1990] further developed this into the optimised dual-submovement model which is consistent with both Schmidt's and Fitts' laws, with the variability of submovements being proportional to the average velocity and that variability leading to the requirement of multiple submovements optimised to minimise the total movement time. The assumption being that subjects move to the target region as quickly as possible, while maintaining a high proportion of target hits. To achieve that, they need to cope with the variability induced by motor noise.

The Iterative Corrective Submovements model proposed by Crossman and Goodeve [1983] is a model where an aimed movement is understood as a series of individual submovements towards the target, each with a constant error and constant duration. Crossman and Goodeve show how Fitts' law can be derived from this model.

In HCI, movement is also often understood as a series of events, such as submovements, and the Crossmann and Goodeve model is widely used. This includes well-known HCI models, such as the work by Card et al. [1986], who introduced their "Goals, Operators, Methods, and Selection rules (GOMS)" framework where a Model Human Processor represented movement as a series of discrete steps. However, Crossman and Goodeve [1983] also presented an alternative continuous explanation of aimed movements that predicts the position of the hand at any point in time, during the movement. ${ }^{1}$

\subsection{Intermittent Control}

Feedback control involves applying a control signal to a dynamic system, based on observations which are combined into an estimate of the system's state ("sensing"), and a feedback law, cf. Figure 1. In the traditional CC paradigm (bottom left in Figure 1), the control signal is continuously adjusted using continuously observed information. In contrast, IC, as introduced in Gawthrop et al. [2011, 2015] and Gawthrop and Wang [2009], and shown in the bottom right of Figure 1, employs an event-trigger to switch between closed- and open-loop configurations, using feedback at only specific moments in time to generate an control trajectory that is then applied during the openloop interval, where there is no feedback.

There are strong theoretical and practical modelling motivations for the use of IC in human motor control. It has recently been shown that refractoriness can also be observed in more complex human control tasks [van de Kamp et al. 2013], and that a control strategy which applies feedback only intermittently can successfully model this phenomenon [Loram et al. 2012]. The physiological basis for muscle activity in human motor control is also a motivation for the use of intermittent models, and provides insight into the source of variability in human control of input devices [Gollee et al. 2017]. Intermittency can serve as a mechanism to modulate the relationship between exploration and exploitation or, in other words, the stability and plasticity tradeoff that is critical for adaptation and learning in human behaviour [Loram et al. 2015]. When humans learn to control something new, such as a mouse, they need to build an internal model of the dynamics of what they are controlling. In particular, they need to identify parameters of such an internal model from interaction with the device, i.e., in a closed-loop setting. In any situation where we are trying to identify model parameters from noisy data acquired in a closed-loop setting, there are problems of bias in parameters due to the correlations between input and output induced by the

\footnotetext{
${ }^{1}$ Interestingly, this first-order-lag (1OL) model of movement seems to be less well known in HCI than the Iterative Corrective Submovements model presented in the same paper. The $1 \mathrm{OL}$ assumes that the user is continuously observing the cursor position, and adjusts the velocity of the mouse as a constant fraction of the distance of the cursor to the target. Crossman and Goodeve also show how Fitts' law can be derived from this different model.
} 


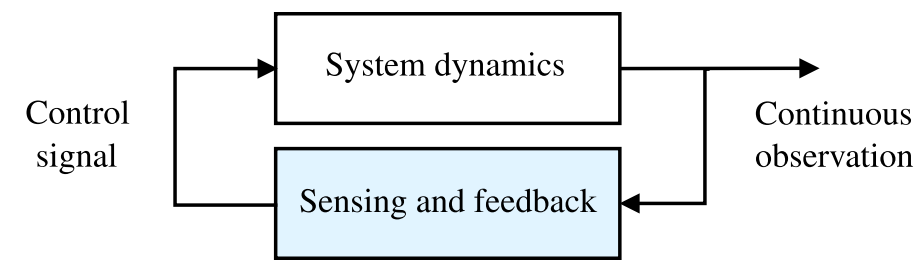

Continuous control

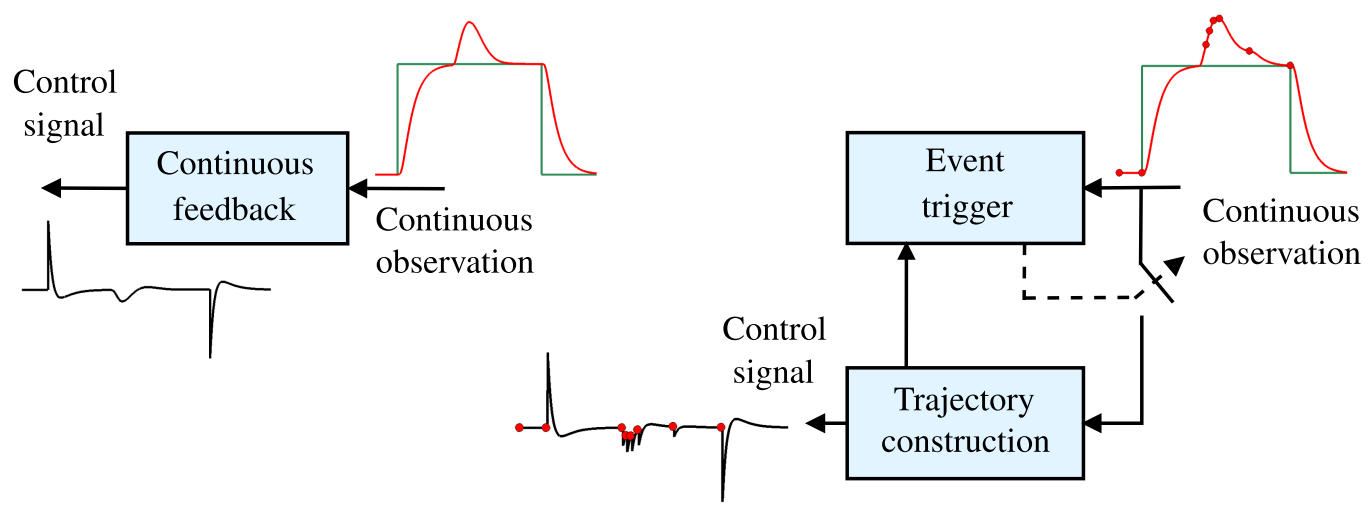

Fig. 1. Feedback control: CC and IC. The top diagram shows a generic control loop: Feedback can be used to control the behaviour of a dynamic system, which involves "sensing" relevant variables to form a representation of the system's state, before generating a control signal based on a feedback control law. In CC (bottom left block diagram), the control signal (time-series in black) is generated continuously, based on observations of the system (time-series in red). In IC (bottom right), feedback information from continuous observations is only used intermittently (determined by an event trigger) to reset an open-loop control signal trajectory. The instances when feedback is used in IC are indicated by circles on the red and black time-series, representing the system output and the control signal, respectively. The control signal trajectories between two consecutive red circles (or events) are generated open-loop, i.e., without the use of feedback.

feedback [Forssell and Ljung 1999]. The intermittent breaking of the feedback loop in IC allows model parameter fitting without the correlated noise from feedback affecting the estimates.

Previous applications of IC in human motor control were mostly interested in how humans can move their bodies from a physiological perspective, so aimed movements were mostly investigated with the bare hand, or with absolute control devices such as joysticks. This article is the first application of the formal IC framework to mouse movements in an HCI context. The mouse provides only a relative mapping between mouse and pointer movements, considerably complicating the control mapping. For these reasons, pointing with the mouse has been investigated very rarely in the field of human motor control. In contrast, mouse movements are at the heart of the field of $\mathrm{HCI}$, looking at situations where humans control the state of a computer system, via potentially complicated mappings.

\subsection{Control of Computers}

Traditionally, HCI is often presented as communication of information between the user and computer, and has used information theory to represent the bandwidth of communication channels into and out of the computer via an interface, but this does not provide an obvious way to measure the communication, or whether the communication makes a difference. Information theory 
alone is not sufficient as a framework for modelling HCI. ${ }^{2}$ Humans often want to control some aspect of the world (e.g., the temperature in a room, or the volume of a music player) via a computer, or change the state of a computer in its own right. In either case, we have a dynamic feedback loop which passes through a computer, where in order to communicate the simplest symbol of intent, we typically require to move our bodies in some way that can be sensed by the computer, often based on feedback while we are doing it. Our bodies move smoothly through space and time, so any communication system is going to be based on a foundation of CC. However, inferring the user's intent is inherently complicated by the properties of the control loops used to generate the information-intention in the brain becomes intertwined with the physiology of the human body and the physical dynamics and transducing properties of the computer's input device, as well as the brain's own predictions of these aspects. We therefore have sensitivity to both variability of human motor control execution, and need to take user predictions about their own behaviour into account, highlighting the role of the model-predictive IC methods explored in this article. ${ }^{3}$ MurraySmith [2018] gives an overview of the role of control theory in the study of HCI, and Jagacinski and Flach [2003] provide a readable introduction to control and dynamics concepts for non-engineers.

Control theory provides an engineering framework which is well suited for analysis of closedloop interactive systems. This can include properties such as feedback delays, sensor noise (see e.g., Trendafilov and Murray-Smith [2013]), or interaction effects like "sticky mouse" dynamics, isometric joystick dynamics [Barrett et al. 1995], magnification effects, inertia, fisheye lenses, speed-dependent zooming, all of which can be readily represented by dynamic models. The use of state space control methods was explored in the document zooming context in Eslambolchilar and Murray-Smith [2004, 2006, 2008], Kratz et al. [2010], and Quinn et al. [2013] reviewed the challenge of optimising touch scrolling transfer functions and used a robot arm to identify the dynamics of commercial products. Examples of the use of dynamic models in interactive dynamic scrolling systems are now widespread in commercial systems, and examples in the academic literature include Cho et al. [2007] and Williamson et al. [2007]. Highlighting the relevance of knowledge of variability in user movements, Quinn and Zhai [2018] used control models to understand how input trajectories associated with words entered into gesture keyboards are likely to vary.

The control perspective can also inspire unusual approaches to interaction, such as motion pointing interfaces which infer the user's intent based on detection of control behaviour, as originally developed by Williamson and Murray-Smith [2004] and built on by Fekete et al. [2009] and, via eye tracking, in a number of applications described in Velloso et al. [2017]. Improved estimation of variability in closed-loop control behaviour would increase the performance of many of these approaches significantly, as they rely on measurement of the divergence of distributions. Motion pointing requires a good model of the user's ability to perform certain actions. Recent interest in the relevance of detailed inverse biomechanical simulation in HCI includes Bachynskyi et al. [2015] and Bachynskyi [2016], while Fischer et al. [2020] explore forward biomechanical simulation to simulate pointing movements.

In Müller et al. [2017], we presented and compared several manual control models of mouse pointing identified from the same mouse movement data used in this article. These models are generative, estimating not only movement time, but also pointer position, velocity, and acceleration on a moment-to-moment basis. The CC models tested captured some of the important dynamic

\footnotetext{
${ }^{2}$ Julien Gori has been updating the information theoretic analysis of Fitts' law tasks in Gori [2018] and, in Gori et al. [2018], highlighted the potential benefits of including feedback in the analysis.

${ }^{3}$ Note the similarity to issues in the foint cognitive systems community, where Hollnagel [1999] and Hollnagel and Woods [2005] argue that we need to focus on how the joint human-computer system performs, not on the communication between the parts.
} 
characteristics, but did not explain the variability shown in the data, which led to a poorer fit for low ID targets. The models included continuous second-order dynamics models, and Costello's surge model, which has a switching characteristic.

The switching characteristic separates the movement into an open-loop initial surge phase and a later continuous current control phase. This switching behaviour has also been modelled by Aranovskiy et al. [2016] and further in Aranovskiy et al. [2020]. Based on the optimised dualsubmovement model and the surge model, they develop a two-phase model that takes into account non-linear pointing transfer functions (PTFs) which map from mouse movement to cursor movement. While the ballistic movement phase only has access to proprioceptive feedback for control, the following corrective movement phase is guided visually from the pointer position which is mediated by the PTF. The switch between ballistic and corrective movement phase happens at a predefined distance from the target after a transition period with zero acceleration. Aranovskiy et al. derive the exponential stability of the model under some mild assumptions about the PTF, and validate the model against experimental data of reciprocal pointing in the case of a constant gain function. In contrast to the IC model presented in this article, the model of Aranovskiy explicitly models pointing with a possibly non-constant PTF. On the other hand, the IC model explicitly models intermittency of the control process, the observer and predictive capability of the human, the dynamics of the NMS, as well as noise and delay in the nervous system. Being based on physiological principles of motor control, the IC model has the potential advantage that it is applicable to a range of movement tasks other than mouse movement.

The Intermittent Click Planning Model (ICP) of Park and Lee [2020] describes the process by which users plan and execute optimal click actions, from which the model predicts the pointing error rates for target tracking tasks. Their ICP model assumes that the user is an intermittent controller, following on from the intermittent Basic Unit of Motor Production model described in Bye and Neilson [2008]. The main difference between our approach and the BUMP model is that in this article, we use an event-trigger, leading to a variable distribution of open-loop intervals, whereas the BUMP model is based on a constant intermittent interval. Physiological evidence suggests that the open-loop interval is not constant, so an event trigger should be the more reasonable explanation from Gawthrop et al. [2014]. The ICP model also assumes that the user is a statistical encoder that makes optimal use of the externally provided information that allows for estimation of click timings. More complex threshold functions for IC are an interesting opportunity for future research.

\section{OVERVIEW OF THE IC MODEL}

This section provides an explanation of the main elements in IC with an emphasis on human motor control and integrates them in the form of a unified control model. The purpose is to relate the function of fundamental features of human control in pointing and tracking tasks with classical control theory concepts, while highlighting the differences between IC and a CC point of view.

In this context, the flow of information is represented by signals (such as the pointer position or motor control signals within the user) that are used as inputs to specific systems (such as the computer or the biomechanics of the user), and depending on the role of the system, different outputs are generated. In human motor control and HCI, there are many operations involved in classic tasks that have a direct representation in control theory; for instance, for pointing tasks where the goal is to reach a target using a pointer device of some sort, the target can be seen as a reference signal that should be introduced to the model [Müller et al. 2017]. This signal allows the calculation in real-time of the error between the target position and the pointer position. In most systems, the goal is to reduce this error as much as possible and a common way to achieve this is by using feedback, which means that the output signal delivered by the system we are trying 
to control is used as an input to a controller that computes and applies a correcting signal to the same system. For human in-the-loop systems, this process happens when the system output, such as the display content, is sensed, processed, and integrated via our sensory stream, to form an internal representation of the task in the brain [Shadmehr and Mussa-Ivaldi 2012; Wolpert et al. 1998], meaning that the central nervous system (CNS) uses a combination of prior and present sensory information to estimate internal and external states that are relevant to the task [Bays and Wolpert 2007], such as pointer position and velocity. This internal representation provides the capability to predict how the system output will behave in the future based on its current state, while cancelling the effects of common delays that affect the transmission of these signals through our neural pathways [Gawthrop et al. 2011; Miall et al. 1993] or within the computer, to then combine all the available information in order to generate control commands, which cause the muscles to contract in a way to achieve the desired effect.

The aforementioned process can be defined by three main components: (1) the system that describes the evolution in time (time-series) of the controlled variable or output, known simply as the system, which includes the computer hard-and software; (2) a high-level controller in charge of sensing outputs, predicting future states and generating control inputs which are implemented in the users' CNS; and (3) the NMS of the user which provides the necessary correction, normally as a force or torque applied to a pointing mechanism, such as a joystick or a computer mouse.

A continuous flow of signals between a system and its user through any given interface is an example of a continuous interaction model where the system output is continuously being sensed and, consequently, control commands are generated as a result of this continuous stream. Although this continuous model has been the dominant framework to explain motor control, there are situations where this closed-loop system is not always continuous, for instance, when the contact between the hand and the input device to the system is interrupted [Loram et al. 2011], or when the system output is not available for measurement. These situations yield a system that is intermittently continuous. However, a third source of intermittent behaviour arises as a result of the refractory period that is observed frequently in reaction tasks [Telford 1931], which implies that even with a continuous flow of information in the form of feedback, humans respond to a succession of sufficiently rapid stimuli in a serial way, starting the response for new stimuli only after finishing the processing and execution of the previous one, thus evolving for short periods of time in an open-loop configuration [Craik 1947; Navas and Stark 1968]. This serial-ballistic operation mode is at the core of the IC model that is presented in this article for HCI tasks, providing a flexible architecture that includes continuous interaction as a special case of a more general framework.

\subsection{The IC Framework}

Let us introduce the general IC model by presenting the CC counterpart first using a block diagram, which is a common graphical representation of the structure and purpose of a control system. In Figure 2(A), a continuous interaction model is shown in the context of target tracking using a pointer device (e.g., computer mouse or joystick). The arrows represent signals travelling through the loop that act as inputs and outputs to the different systems in the model (depicted as white boxes with solid black borders). The user is represented by the light blue coloured box containing two subsystems, the Human-Computer system dynamics box (in grey dashed lines), which includes the dynamics of the arm and hand in relationship to the pointer device, and a high-level continuous controller shown in a red coloured box composed of three blocks: Sensory integration, Prediction, and Control gains. The Human-Computer system dynamics box contains the group of variables that the user attempts to control and it receives a correcting input signal that the CC system generates. The mouse velocity measured by the mouse sensor is transferred to the pointer velocity via a possibly non-linear PTF [Casiez and Roussel 2011; Casiez et al. 2008]. The output of this 


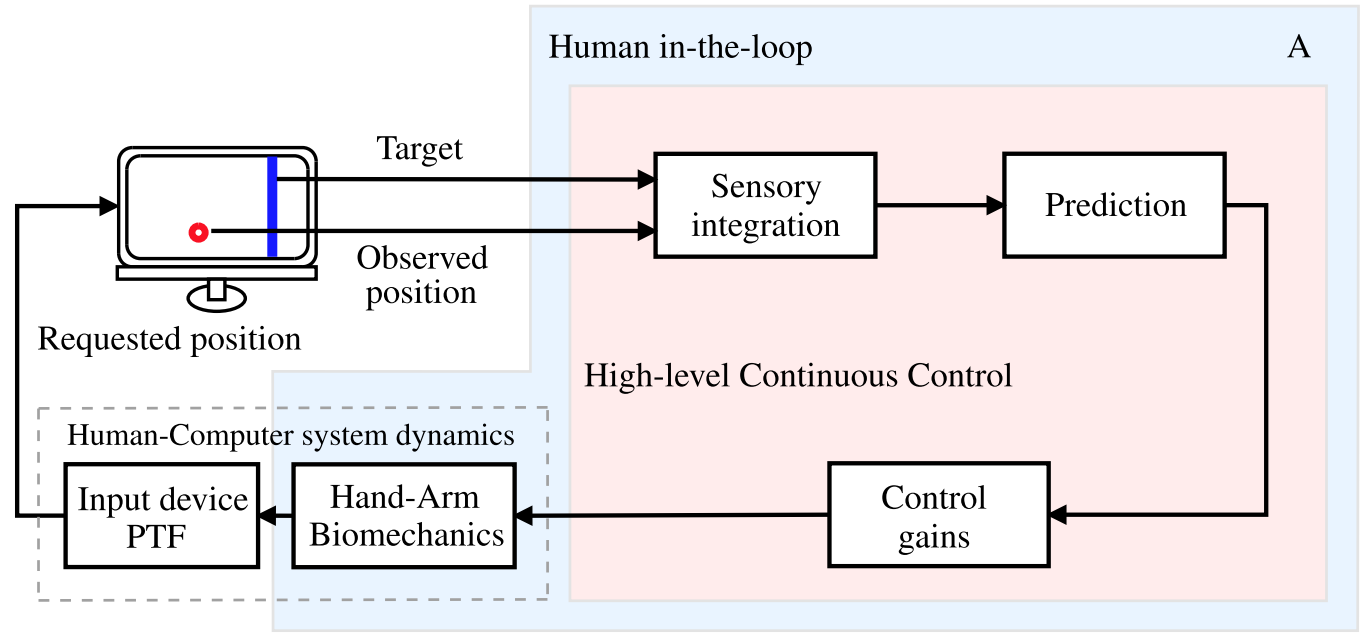

Human in-the-loop

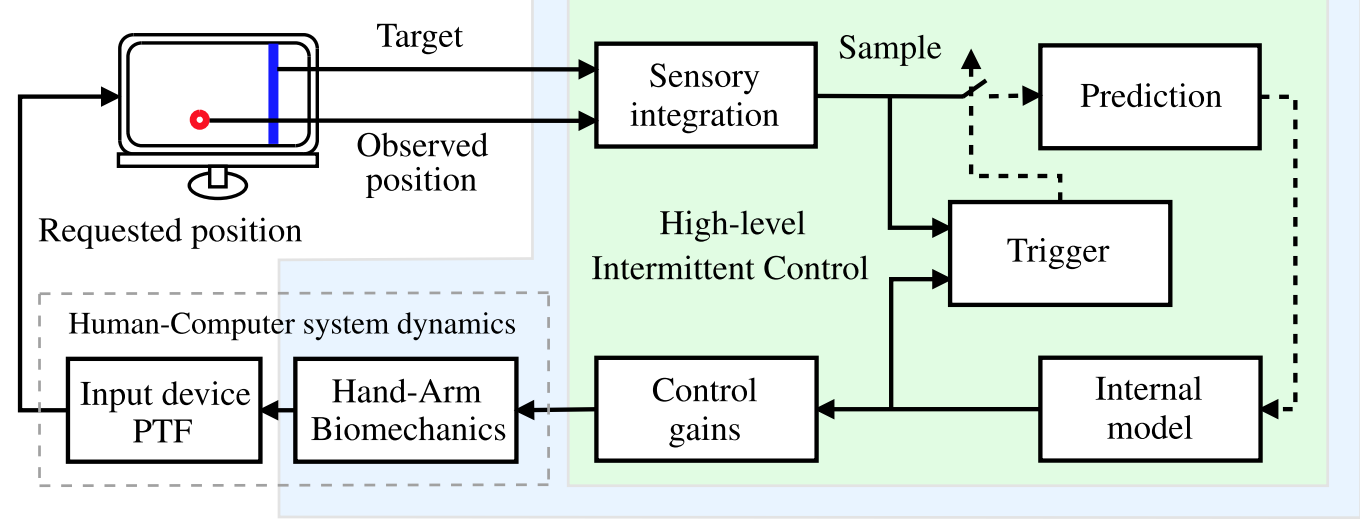

Fig. 2. CC (above) and IC (below) frameworks in the context of a general $\mathrm{HCl}$ task. The black solid lines represent signals going in and out the different systems continuously, whereas the dashed lines indicate that they are active intermittently. (A) CC performs three tasks: sensory integration, prediction, and input generation modulated by a set of Control gains. (B) IC (green box) includes an Internal reference model, a Trigger, and Sampling mechanism. Both models take into account the human-computer system dynamics (grey dashed-line box) as the combination of two separate systems: the Hand-Arm Biomechanics and the Input Device PTF. The pointing task is to bring the displayed pointer (red circle) to the Target (blue vertical bar). The user is represented in blue.

block (e.g., pointer position on a display) is then sensed via visual feedback and used to generate a new control action. The CC system as a whole is in charge of reducing the error between the target signal (desired pointer position) and the output (current pointer position) by combining sensory streams and intended targets to form an internal model of the task at hand. It is argued that the brain uses some form of model based Prediction to better understand the consequences of our commands during movement and to diminish the effect of slow transmission pathways [Bhushan and Shadmehr 1999; Gawthrop et al. 2011; Gawthrop and Wang 2008; Miall et al. 1993], this concept is represented by the block that follows the system integration process. The last stage 
involves the selection and computation of an appropriate high-level Control signal which is applied continuously.

The IC model, shown in Figure 2(B), incorporates the same elements of the CC model with the addition of a Trigger mechanism within the user's CNS that has the fundamental role of opening or closing the loop right after the output of the sensory integration process; this effectively samples the output and relevant states of the system. Regulating the flow of information at this point allows the user to achieve continuous measurement of the output variables while applying control commands intermittently. This mechanism can replicate refractoriness by enforcing a minimum amount of time in which the system would evolve in an open-loop configuration. In addition to this, the controller could extend these open-loop intervals beyond this minimum time by comparing the states of an internal dynamical model that represent the task and the sensory outputs coming in the form of feedback. This concept is discussed in the following section.

\subsection{Event Generation Based on an Internal Model}

The generation of control commands by the user in IC (shown in Figure 2(B)) can be seen as the interplay of an Internal model and a set of Control gains within the CNS of the user that modulate the input signal that is applied to the system. The internal model is a central feature of IC and serves as reference in terms of performance, using a dynamical model to mimic the behaviour of a continuous delay-free version of the system [Gawthrop et al. 2015]; therefore, in the ideal scenario of control inputs and system outputs that are not corrupted by motor or observation noise, respectively, or affected by external disturbances, the states of this internal model should match the ones generated by the sensory integration process.

On the other hand, if the observations predicted by the user differ from the actual observations, it means that there are unaccounted disturbances which produce a deviation from the reference internal model. It is possible to use this source of error to decide if the sampling mechanism should stay open or to close it, which would update the Prediction stage and, consequently, the internal model itself with measured states. During the time in which the sampling mechanism is open, the internal model is evolving on its own and producing states continuously without using available feedback information, the fact that this process happens continuously ensures that a control signal is always going to be computed. In the case of a discrete movement, such as the initial ballistic submovement towards the target (surge), the motor signals in charge of the execution are planned in advance and executed ballistically. In order to plan such a ballistic movement, the internal model is necessary. One way to determine the mode in which the overall control loop should operate (open- vs closed-loop) is to compare the error between the sensed output and the version of the output generated by the internal model against a fixed value, establishing a triggering threshold. If the error is larger than the threshold then the controller should rely on feedback to reduce it (trusting sensed information more). This constant comparison carried out by the trigger mechanism generates events, which define the moment in time when the error overcomes the threshold value. This particular implementation of IC is often referred to as Event-Based or Event-Driven IC [Gawthrop and Wang 2009]. When an event is created, the feedback loop is closed only for a small instant and then reopened, which forces the controller to stay open-loop for a minimum amount of time before feedback can be used again due to new events.

The IC model shown in Figure 2(B) was conceived from a computational perspective as an additional layer of an existing continuous model proposed by Kleinman, which used a linear optimal controller to approximate the response of a human operator in manual control tasks [Kleinman et al. 1970; Kleinman 1969]. The previously mentioned elements of sensory integration, prediction, and control generation formed the basis for Kleinman's model, linking physiology concepts with control theory, while providing solid basis for IC. 


\subsection{Human Variability in Motion}

Variability is a distinctive element of human motion and human-in-the-loop systems. A human generating a control action repeatedly over a system with a defined set of external perturbations, would generate output responses that are slightly different from each other. Physiologically, variability is generated at any level of the motor pathway and it is normally attributed to a combination of factors: (1) neural spike initiation-propagation, as well as synaptic transmission and muscle activation, all rely on biophysical and chemical events which are stochastic in nature [de C. Hamilton et al. 2004; Faisal and Laughlin 2007; Faisal et al. 2008; Jones et al. 2002] and (2) variation in movement planning and decision making processes happening in the CNS [Dhawale et al. 2017; Gollee et al. 2017].

From a computational point of view, the variability of human movements has been explained by adding motor and observation noise to a continuous representation of the overall closed-loop system [Levison et al. 1969; Van Der Kooij and De Vlugt 2007; Van Der Kooij and Peterka 2011], implying that these differences are the result of a linear process in combination with a stochastic component, introduced by the random noise. However, there is experimental evidence that shows how manual control is subject to a refractory process [Loram et al. 2011, 2014; Miall 1986; Navas and Stark 1968], strengthening the view of sustained sensorimotor control as a discrete sequence of open-loop intervals combined with feedback instances of sensory information. It has also been proposed that a triggering mechanism is behind the decision of closing or opening the feedback loop, where the timing of each event is decided by error signals crossing a threshold value.

Results from a visuo-manual tracking task [Gollee et al. 2017; Loram et al. 2011], in which the participants used a joystick to control the position of an unstable dynamical system (represented by a dot on a screen), showed that an intermittent-predictive controller is able to explain both the linear and non-linear components of the output response at both excited and non-excited frequencies, without adding any coloured noise. Whereas a continuous predictive controller requires the addition of a separate motor noise profile for each of the conditions in the experiment to adequately describe the output response. These findings suggest not only that the aperiodic triggering mechanism is a plausible hypothesis to explain manual tracking but also that the intermittency is directly related to the observed variability.

The constant switching between open- and closed-loop evolution introduces other benefits, such as the possibility to clearly distinguish the effects of the applied input commands, the external disturbances, and the natural dynamics of the system [Loram et al. 2011]. Moreover, the variability that is introduced by the aperiodic triggering process of IC plays an important role in adaptation and learning by regulating the tradeoff between exploration and exploitation [Loram et al. 2015]. It has been shown that higher rates of movement-to-movement variability in motor output predict faster learning rates for specific motor control tasks. This suggests that regulating variability according to the environment and the task at hand might be an essential feature of the CNS to promote either fine control when precision is needed or broad exploration to accelerate adaptation and learning [Wu et al. 2014].

\section{THE IC MODEL}

In this section, a more detailed explanation of IC is given, with an emphasis on describing the main elements of the framework more formally, from a control theory perspective, to support a reader wishing more insight into the model structures, rationale, and behaviour. While the theory of IC in this section is fundamental to understand the details of the framework as a control methodology, the main results of this article are largely accessible if skipped at a first reading. The high-level IC described in Figure 2(B) in the context of a pointing task, is reintroduced using a more general 


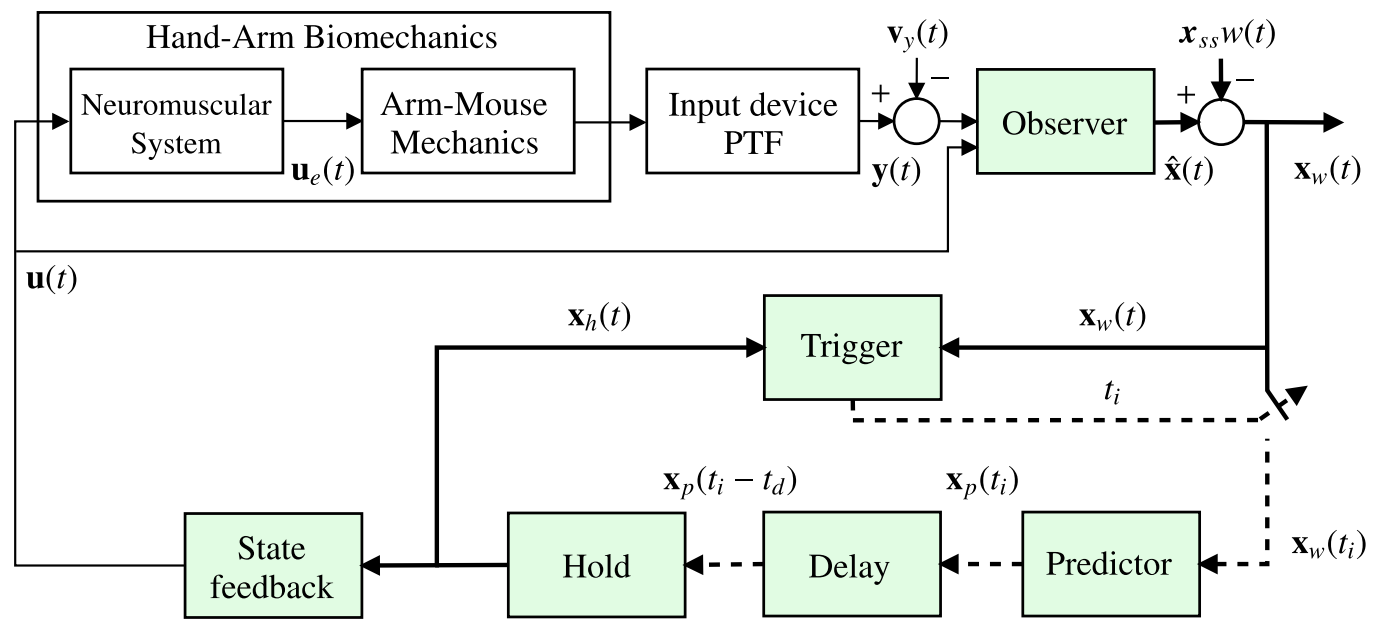

Fig. 3. Diagram of the intermittent controller within the CNS of the user. The hold states $\mathbf{x}_{h}$ are compared with the state-estimates $\mathbf{x}_{w}$ in the trigger block. If the difference exceeds a predefined threshold, then the block creates events at times denoted by $t_{i}$. The hold states $\mathbf{x}_{h}$ are used to generate the control signal $\mathbf{u}$ during the open-loop period, and it is reset only at times $t_{i}$ by the predictor block. The dashed lines represent signals that are defined only at $t_{i}$. The thick lines/arrows of the diagram represent vector signals, whereas the thin ones represent scalar versions, for the single-input single-output case. This figure is based on the representation given in Gawthrop et al. [2011]. The Hand-Arm Biomechanics block contains the NMS which generates the force that is applied to the input device and the Arm-Mouse Mechanics block that translates it into input to the PTF.

approach in Figure 3, which is, in turn, based on the versions presented in Gawthrop et al. [2011, 2015] and Gawthrop and Wang [2007]. Together, the Hand-Arm Biomechanics and Input device PTF blocks from Figure 3 represent a mathematical model of the neuromuscular dynamics of the user and the user interface. Although user interface dynamics can, in principle, be highly non-linear, e.g., via non-linear PTFs, for simplicity, in the following, we assume that the overall dynamics can be expressed as a linear state-space model of order $n$ that corresponds to the case of constant gain in pointing, and which can be expressed as follows:

$$
\begin{aligned}
\dot{\mathbf{x}}(t) & =\mathrm{Ax}(t)+\mathrm{Bu}(t) \\
\mathbf{y}(t) & =\mathbf{C x}(t),
\end{aligned}
$$

with $\mathbf{x}(0)=\mathbf{x}_{0}$ being an initial condition. $\mathbf{x} \in \mathbb{R}^{n}$ corresponds to the system state such as pointer position and velocity, and muscle activation, $\mathbf{y} \in \mathbb{R}^{n_{y}}$ corresponds to the output such as pointer position on the display, and $\mathbf{u} \in \mathbb{R}^{n_{u}}$ corresponds to the input such as muscle excitation. $t$ represents continuous time. A is an $n \times n$ matrix representing how the system evolves without control input, $\mathrm{B}$ is an $n \times n_{u}$ matrix, representing the impact of control signals such as muscle excitation on the system, and $\mathrm{C}$ is an $n_{y} \times n$ matrix, representing how the system state maps to the users' observation, such as pointer position on the display.

Two concepts from control theory are potentially generally useful for HCI models. These are the observer and the predictor. The control goal of the user is to bring the system output $y(t)$ as close as possible to the target signal $w(t)$ (e.g., the pointing target). The system integration process mentioned in Figure 2(B) which combines multiple sensory channels, can be interpreted as an estimation problem. The central issue is that the control problem might not be solvable from the observations alone, if the true state is unknown. For example, in pointing, the user can observe 
only the pointer position. If the pointer velocity, muscle activation, and so on cannot be observed directly, they need to be estimated in order to enable the user to control the system.

Observer: To solve this issue, the input $\mathbf{u}(t)$ and the output $\mathbf{y}(t)$ are fed to a linear observer, which is a dynamical model based on Equation (1), that reconstructs the full state of the system $\hat{\mathbf{x}}(t)$ using only output measurements and information on the input that is being applied. Once the target signal $w(t)$ has been introduced as $\mathbf{x}_{w}(t)=\hat{\mathbf{x}}(t)-\boldsymbol{x}_{s s} w(t)$, the resulting observed states $\mathbf{x}_{w}(t)$ get sampled, this updates the rest of the structures in the feedback loop, only at discrete points in time $t_{i}$.

Predictor: In this implementation, the role of the predictor is to compensate for transmission time-delays which might be present in the feedback loop both from the users neural system and from the computer system, this is done by predicting the future states of the system $\mathbf{x}_{p}\left(t_{i}\right)$ based on the state estimates $\mathbf{x}_{w}\left(t_{i}\right)$ which are defined only when a sample is taken.

The concept of an internal model mentioned earlier in Figure 2(B), is represented in control terms as a generalised hold, which is a dynamical model that mimics the behaviour of the overall closedloop system by continuously generating the state vector $\mathbf{x}_{h}(t)$. The trigger compares the hold states $\mathbf{x}_{h}(t)$ and the observed states $\mathbf{x}_{w}(t)$ to generate a prediction error $e_{p}$. An event is generated by the trigger at $t_{i}$ once $e_{p}$ exceeds a predefined threshold $q$. The hold states $\mathbf{x}_{h}(t)$ are used to compute a state feedback control input $\mathbf{u}(t)$ continuously, which is the signal that drives the NMS producing the final input to the system $\mathbf{u}_{e}(t)$. Notice that both the input $\mathbf{u}(t)$ and the output $\mathbf{y}(t)$ can be affected by motor noise $\mathbf{v}_{u}(t)$ and sensory noise $\mathbf{v}_{y}(t$,$) respectively.$

Before introducing definitions for the hold, the predictor, and the trigger mechanism, it is important to define the different time-frames that are used in IC to accurately distinguish between what happens during the open-loop intervals and the instances of feedback.

\subsection{IC Time-Frames}

IC combines the use of the following time-frames:

(1) Continuous-time $(t)$ represents the time in which the system defined in equation (1) evolves.

(2) Discrete-time $\left(t_{i}\right)$ time instants at which an event is generated, indexed by $i$. The time between event instants is known as the intermittent interval $\Delta_{i}$. The ith intermittent interval can be defined as

$$
\Delta_{o l}=\Delta_{i}=t_{i+1}-t_{i} .
$$

A sampling delay $\Delta_{s}$ can be used to sample the observed states $\mathbf{x}_{w}(t)$ once a fixed-time interval has elapsed after the detection of an event at $t_{i}$. If $\Delta_{s}=0$ then the sample is taken at $t_{i}$.

(3) Intermittent-time $(\tau)$ a continuous variable that is restarted every $\Delta_{i}$ according to

$$
\tau=t-t_{i} .
$$

A lower limit $\Delta_{o l}^{\min }$ can be specified within a given intermittent interval as

$$
\Delta_{i}>\Delta_{o l}^{\min }>0 \text {. }
$$

The lower limit $\Delta_{\text {ol }}^{\mathrm{min}}$, also known as minimum open-loop interval, has been used before to model the psychological refractory period observed in human motor control [Gawthrop et al. 2011].

Based on these definitions, it is now possible to define the model used as the generalised hold, which is a continuous closed-loop approximation of the overall system that relies on the following underlying controller. 


\subsection{Underlying Continuous Controller}

A state-feedback controller for the system in equation (1), with gain $\mathbf{k}$, can be established by implementing the following control-law:

$$
\mathbf{u}(t)=-\mathbf{k x}(t)
$$

Standard procedures such as pole-placement or the linear quadratic regulator (LQR) approach [Goodwin et al. 2001] can be used to obtain the gain $\mathbf{k}$. The resulting closed-loop system, defined by the state vector $\mathbf{x}_{c}$ is

$$
\dot{\mathbf{x}}_{c}(t)=\mathbf{A}_{c} \mathbf{x}_{c}(t)
$$

which depends on $\mathbf{x}_{c}(0)=\mathbf{x}_{0}$ as the initial condition and a closed-loop matrix $\mathbf{A}_{c}$ defined as follows:

$$
\mathrm{A}_{c}=\mathrm{A}-\mathrm{Bk} \text {. }
$$

The target signal $w(t)$ can be introduced by defining the following system:

$$
\begin{aligned}
\boldsymbol{0}_{n \times 1} & =\mathrm{A} \boldsymbol{x}_{s s}(t)+\mathrm{B} \boldsymbol{u}_{s s}(t) \\
\boldsymbol{y}_{s s}(t) & =\mathrm{C} \boldsymbol{x}_{s s}(t),
\end{aligned}
$$

where $\boldsymbol{x}_{s s}, \boldsymbol{u}_{s s}$, and $\boldsymbol{y}_{s s}$ correspond to the steady-state versions of the states, inputs, and outputs, respectively. Solving for $\boldsymbol{x}_{s s}$ and $\boldsymbol{u}_{s s}$ can be done by writing the system as follows:

$$
\left[\begin{array}{l}
\boldsymbol{x}_{s s}(t) \\
\boldsymbol{u}_{s s}(t)
\end{array}\right]=\left[\begin{array}{cc}
\mathrm{A} & \mathbf{B} \\
\mathbf{C} & \mathbf{0}
\end{array}\right]^{-1}\left[\begin{array}{c}
\mathbf{0}_{n \times 1} \\
1
\end{array}\right] .
$$

Thus, the overall control input from Equation (5) can be redefined as

$$
\begin{aligned}
\mathbf{u}(t) & =-\mathbf{k}\left(\mathbf{x}(t)-\boldsymbol{x}_{s s} w(t)\right)+\boldsymbol{u}_{s s} w(t) \\
& =-\mathbf{k x}(t)+\left(\boldsymbol{u}_{s s}+\mathbf{k} \boldsymbol{x}_{s s}\right) w(t) .
\end{aligned}
$$

By defining $\mathbf{r}=\boldsymbol{u}_{s s}+\mathbf{k} \boldsymbol{x}_{s s}$, a simplified expression is obtained:

$$
\mathbf{u}(t)=-\mathbf{k x}(t)+\mathbf{r} w(t) .
$$

The expression in (11) reduces the error between the output $\mathrm{y}(t)$ and the target signal $w(t)$, depending on the value of the gain $\mathbf{k}$. This assumes that the matrices $\mathbf{A}$ and $\mathbf{B}$ are such that the model in (1) is controllable with respect to $\mathbf{u}(t)$, which means that the control input $\mathbf{u}(t)$ has the ability to drive the state-vector $\mathbf{x}(t)$ from its initial condition to any final value in a finite amount of time.

\subsection{State Observer}

In many cases, the system state $\mathbf{x}(t)$ in Equation (1) is not fully available for direct measurement. For example, usually only the pointer position is shown by the computer, but not the pointer velocity. One way to overcome this is to implement a state observer that estimates the portion of $\mathbf{x}(t)$ that is not known using only available information such as the output $\mathbf{y}(t)$, known states, and the current input $\mathbf{u}(t)$. An observer for the system described in Equation (1) can be designed by defining a vector $\hat{\mathbf{y}}(t)$ which contains all the available signals as follows:

$$
\dot{\hat{\mathbf{x}}}(t)=\hat{\mathrm{A}} \hat{\mathbf{x}}(t)+\mathbf{B u}(t)+\mathbf{L}\left[\hat{\mathbf{y}}(t)-\mathbf{v}_{y}(t)\right],
$$

where $\hat{\mathbf{A}}=\mathbf{A}-\mathbf{L} \hat{\mathbf{C}}$ and $\hat{\mathbf{y}}(t)$ is defined as

$$
\hat{\mathbf{y}}(t)=\hat{\mathbf{C}} \mathbf{x}(t) .
$$

Therefore, the matrix $\hat{\mathbf{C}}$ defines which elements from $\mathbf{x}(t)$ are used as inputs for the observer in order to reconstruct the full state. The matrix $\mathrm{L}$ is a design parameter similar to the feedback gain $\mathbf{k}$ in that both of them can be designed via pole-placement or LQR methods. 


\subsection{Generalised Hold}

The generalised hold uses the dynamics imposed by Equation (7) to define the open-loop behaviour in IC. This is achieved by implementing the following state-feedback control-law:

$$
\mathbf{u}(t)=\mathbf{u}\left(t_{i}+\tau\right)=-\mathbf{k x}_{h}(\tau),
$$

where the hold states $\mathbf{x}_{h}$ evolve in the intermittent time $\tau$ according to the following autonomous system:

$$
\dot{\mathbf{x}}_{h}(\tau)=\mathbf{A}_{h} \mathbf{x}_{h}(\tau)
$$

When the observed states are sampled at $t=t_{i}$, the hold states $\mathbf{x}_{h}$ are reinitialised using vector $\mathbf{U}_{i}$ as follows:

$$
\mathrm{U}_{i}=\mathrm{K}_{h} \mathbf{x}_{p}\left(t_{i}-t_{d}\right) \text {. }
$$

Expression (16) takes the predicted states $\mathbf{x}_{p}\left(t_{i}-t_{d}\right)$, which cancel the effect of the time-delay $t_{d}$, to reset the hold state $\mathbf{x}_{h}$ at the start of each intermittent interval

$$
\mathbf{x}_{h}\left(t_{i}\right)=\mathbf{U}_{i}
$$

The square matrix $\mathrm{K}_{h}=\mathrm{I}_{n \times n}$ in Equation (16) is defined as the IC gain. The overall dynamics of the autonomous system in Equation (15) are then determined by matrix $\mathbf{A}_{h}$. If the closed-loop matrix $\mathrm{A}_{c}$ in Equation (7) is used as follows:

$$
\mathbf{A}_{h}=\mathbf{A}_{c}
$$

then the generalised hold becomes a system-matched hold [Gawthrop and Wang 2011] that effectively approximates the dynamics of the closed-loop system defined in Equation (6). The assignment in Equation (18) provides a common ground to compare the estimated states provided by the observer and to those coming from the hold, which can be seen as reference states for the IC framework.

\subsection{Intermittent Prediction}

A predictor can be implemented as a compensation strategy in the presence of time-delays. The following dynamical system can be established during the intermittent time-frame $\tau$ :

$$
\dot{\mathbf{x}}_{p}(\tau)=\operatorname{Ax}_{p}(\tau)+\mathbf{B u}(\tau)
$$

with $\mathbf{x}_{p}(0)=\mathbf{x}_{w}\left(t_{i}\right)$ and evaluated at $\tau=t_{d}$. Combining Equations (19) and (15) yields the following extended system:

$$
\frac{d}{d \tau} \mathbf{X}(\tau)=\mathbf{A}_{p h} \mathbf{X}(\tau)
$$

subject to $\mathrm{X}(0)=\mathrm{X}_{i}$ as the initial condition, and where

$$
\mathrm{A}_{p h}=\left[\begin{array}{cc}
\mathrm{A} & -\mathrm{Bk} \\
0_{n \times n} & \mathrm{~A}_{h}
\end{array}\right] \text {. }
$$

The extended state vector $\mathbf{X}$ from Equation (20) is defined as $\mathbf{X}(\tau)=\left[\mathbf{x}_{p}(\tau) \mathbf{x}_{h}(\tau)\right]^{T}$ during the open-loop interval. At $t_{i}, \mathrm{X}$ takes the following form:

$$
\mathbf{X}_{i}=\left[\begin{array}{c}
\mathbf{x}_{w}\left(t_{i}\right) \\
\mathbf{x}_{p}\left(t_{i}-t_{d}\right)
\end{array}\right] .
$$

The solution of Equation (19) at $\tau=t_{d}$ yields

$$
\mathbf{X}\left(t_{d}\right)=e^{\mathbf{A}_{p h} t_{d}} \mathbf{X}_{i}
$$

From Equation (23), the predicted states $\mathbf{x}_{p}$ can be obtained every intermittent interval using the following expression:

$$
\mathbf{x}_{p}\left(t_{i}\right)=\mathbf{E}_{p p} \mathbf{x}_{w}\left(t_{i}\right)+\mathbf{E}_{p h} \mathbf{x}_{h}\left(t_{i}\right)
$$


where the matrices $\mathrm{E}_{p p}$ and $\mathrm{E}_{p h}$ of dimension $n \times n$, are partitions of the $2 n \times 2 n$ matrix $\mathrm{E}$ defined as

$$
\mathrm{E}=e^{\mathrm{A}_{p h} t_{d}}=\left[\begin{array}{cc}
\mathbf{E}_{p p} & \mathbf{E}_{p h} \\
\mathbf{E}_{h p} & \mathbf{E}_{h h}
\end{array}\right] .
$$

Both $\mathbf{E}_{p p}$ and $\mathbf{E}_{p h}$ can be obtained offline.

\subsection{Event Detection}

IC produces an aperiodic sequence of events, determined by the error between the hold states $\mathbf{x}_{h}$ and the closed-loop observer state $\mathbf{x}_{w}$ as follows:

$$
e_{x}=\mathbf{x}_{h}(t)-\mathbf{x}_{w}(t) \text {. }
$$

An event is generated when $e_{x}$ is greater than a predefined threshold $q$, according to the following quadratic switching function:

$$
e_{x}^{T}(t) Q_{t} e_{x}(t)>1
$$

where $\mathrm{Q}_{t}$ is a positive semi-definite matrix that defines which states are considered in order to detect events and thus trigger the use of feedback. For instance, an IC triggering on the error of a two dimensional state vector would implement the next switching function

$$
\left(\frac{e_{x_{1}}}{q_{1}}\right)^{2}+\left(\frac{e_{x_{2}}}{q_{2}}\right)^{2}>1,
$$

with a matrix $Q_{t}$ containing the following values in its diagonal:

$$
\mathrm{Q}_{t}=\left[\begin{array}{cc}
\frac{1}{\left(q_{1}\right)^{2}} & 0 \\
0 & \frac{1}{\left(q_{2}\right)^{2}}
\end{array}\right]
$$

This particular choice of the event detection mechanism allows to set different threshold values for specific states and to use different combinations of them.

\section{EXPERIMENT SETUP AND DATASET}

The results shown in this article are based on the Pointing Dynamics Dataset that was collected from a mouse pointing experiment described in Müller et al. [2017]. The IC framework from the previous sections is used to identify the parameters of a standard intermittent controller. The full dataset is publicly available. ${ }^{4}$ In the aforementioned experiment, 12 participants were asked to control a white pointer that was shown on a screen. The pointer reflected the changes in the $x$ dimension of the mouse exclusively and it was restricted to move only horizontally.

The task consisted of clicking a number of one-dimensional targets that were displayed in sequence. During a block of trials, the active target was presented in a different color compared to the previous target, and the distance between them, as well as the width of the target, stayed constant throughout the block. Each block uses a specific combination of distance and width; a total of eight combinations were applied for each subject: (1) distance of $212 \mathrm{~mm}$ and widths of 0.83 , $3.32,14.1$, and $70.6 \mathrm{~mm}$ and (2) distance of $353 \mathrm{~mm}$ and widths of 1.38, 5.54, 23.5, and $118 \mathrm{~mm}$. Fitts' law [Fitts 1954] states that the time required to move to a specific target area $M T$ is a function of both the distance to the target $D$ and the size of the target $W$ as follows:

$$
M T=a+b I D
$$

where the ID is

$$
I D=\log _{2}\left(\frac{D}{W}+1\right)
$$

\footnotetext{
$\overline{{ }^{4} \text { http://joergmueller.info/controlpointing/. }}$
} 
which is known as the Shannon formulation [MacKenzie 1992]. Based on Equation (31) and the selected combinations of distance between targets and target width, the resulting ID for both 212 $\mathrm{mm}$ and $353 \mathrm{~mm}$ conditions are 8, 6, 4, and 2. Therefore, each participant had to complete a full block of trials per ID for the two distance conditions. The experiment blocks were designed to have 102 trials each, divided into 22 trials for training and 80 for the rest of the task.

The participants were asked to stay below a 5\% error rate while clicking on every target as quickly as possible. A beep sound would indicate the user if the click was made outside the target area, triggering the appearance of the next target on screen for that particular block. The users were not asked to repeat failed trials (missed targets). All participants completed a training phase of 22 trials for each block before starting the experiment and had a short break between to rest between blocks.

\section{MODEL IMPLEMENTATION AND CONTROLLER DESIGN}

The intermittent controller shown in Figure 3 was implemented in Matlab ${ }^{5}$ using the Control Systems Toolbox to generate appropriate transfer function and state-space representations of the human dynamics and the system to be controlled. The block labelled as NMS in Figure 3 was implemented as a second-order system with time-constants of $50 \mathrm{~ms}$ [Milner-Brown et al. 1973],

$$
G_{n m s}(s)=\frac{1}{(0.05 s+1)^{2}},
$$

where $G(s)$ is the transfer function in the Laplace domain. This transfer function was converted to its equivalent state-space system in the form of equation (1) with

$$
\mathbf{A}_{n m s}=\left[\begin{array}{cc}
-20 & 20 \\
0 & -20
\end{array}\right], \quad \mathbf{B}_{n m s}=\left[\begin{array}{c}
0 \\
20
\end{array}\right], \quad \mathbf{C}_{n m s}=\left[\begin{array}{ll}
1 & 0
\end{array}\right] .
$$

Similarly, the block shown in Figure 3 as Arm-Mouse Mechanics was established as a double integrator in the state-space representation of Equation (1) with

$$
\mathbf{A}_{s y s}=\left[\begin{array}{ll}
0 & 1 \\
0 & 0
\end{array}\right], \quad \mathbf{B}_{s y s}=\left[\begin{array}{l}
0 \\
1
\end{array}\right], \quad \mathbf{C}_{s y s}=\left[\begin{array}{ll}
1 & 0
\end{array}\right],
$$

where the associated state vector is $\mathbf{x}_{\text {sys }}(t)=\left[\mathbf{x}_{\text {pos }}(t) \mathbf{x}_{v e l}(t)\right]^{T}$, composed of the pointer position $\mathbf{x}_{\text {pos }}(t)$ and velocity $\mathbf{x}_{\text {vel }}(t)$. This assumes that the output of the NMS, depicted as $\mathbf{u}_{e}(t)$ in Figure 3 , is a force generated by the participant, applied to a unit mass in a low friction environment, and that the output of the overall system, $y(t)$, is the position of the mass, which in this case is equivalent to the position of the pointer on the screen. The combination of Equations (33) and (34) yields a fourth-order system based on the full state vector $\mathbf{x}(t)$, which is then used to design the overall controller.

The feedback gain vector $\mathbf{k}$ shown in Equation (5) was designed using optimal control via the LQR design method [Goodwin et al. 2001], to ensure that the closed-loop matrix $\mathbf{A}_{c}$, which defines the behaviour of the generalised hold in IC (18), has stable eigenvalues. This involves the minimisation of the LQR cost function

$$
J_{L Q R}=\int_{0}^{\infty}\left[\mathbf{x}(t)^{T} \mathbf{Q}_{c} \mathbf{x}(t)+\mathbf{u}(t)^{T} \mathbf{R}_{c} \mathbf{u}(t)\right] d t,
$$

and the solution of its associated algebraic Ricatti equation. Both $\mathbf{x}(t)$ and $\mathbf{u}(t)$ in Equation (35) are weighted by design matrices $Q_{c}$ (an $n \times n$ diagonal matrix that must be positive semi-definite) and $\mathbf{R}_{c}$ (an $n_{u} \times n_{u}$ diagonal positive definite matrix, where $n_{u}$ corresponds to the number of inputs in

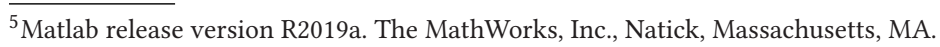


the system). The diagonal nature of $\mathbf{Q}_{c}$ is in fact a design choice, which is based on the assumption of not having crossed-term effects between the states. Using the same LQR method on the dual problem of state estimation, the closed-loop observer gain matrix $\mathrm{L}$, can be computed to force the estimation error to vanish asymptotically, by choosing a design matrix $\mathbf{Q}_{o}$. The observation matrix in Equation (13) was defined as $\hat{\mathrm{C}}=\left[\begin{array}{lll}1 & 0 & 0\end{array}\right]$, which means that only the first state of the state vector $\mathbf{x}(t)$, corresponding to the pointer position $\mathbf{x}_{\text {pos }}(t)$, is used by the observer to estimate the rest to the unknown states. In the context of this work, matrices $Q_{c}$ and $Q_{o}$ were defined as parameters to be optimised, whereas $\mathbf{R}_{c}$ was fixed for the entire process with a value of 1 (in this case, it is a scalar since there is only one input to the system, $n_{u}=1$ ). The time-delay $t_{d}$ remained constant at $0.01 \mathrm{sec}$ for all participants and conditions. Note that the effective time-delay is a combination of the constant $t_{d}$ and the optimised threshold $q$ and minimum open-loop interval $\Delta_{o l}^{\min }$.

To detect events, a threshold $q$ was applied only to $\mathbf{x}_{\text {pos }}(t)$. However, the actual value of $q$ was obtained via the optimisation process. As suggested in Gawthrop and Wang [2009], the use of a disturbance observer is recommended to reduce the effect of zero mean constant disturbances $\mathbf{d}(t)$ at the input; therefore, an integrator is normally used to account for them and compensate accordingly.

\section{OPTIMISATION APPROACH}

Based on the systems (33) and (34), an optimisation procedure was used to fit a set of controller parameters to the experimental data. The following parameters were identified as a result of the optimisation process: the LQR design matrices $Q_{c}$ and $Q_{o}$, the prediction error threshold $q$ for triggering purposes, the minimum open-loop interval $\Delta_{o l}^{\mathrm{min}}$, and a mismatch gain $\mathrm{A}_{p}=1-p$.

The purpose of $\mathbf{A}_{p}$ is to model the cases where the control input that is applied to system is different by a fixed amount from what it should be by design. This is implemented as follows: from Figure 3, the output of the NMS $\mathbf{u}_{e}(t)$, which serves as the input to the system in Equation (34), is multiplied by a quantity $p$, resulting in

$$
\dot{\mathbf{x}}_{\text {sys }}(t)=\mathbf{A}_{\text {sys }} \mathbf{x}_{s y s}(t)+\mathbf{B}_{\text {sys }} \mathbf{u}_{\text {sys }}(t),
$$

where $\mathbf{u}_{s y s}(t)=p \mathbf{u}_{e}(t)$. When $p=1$, the full input is applied. A mismatch is generated when $p$ is different from 1 . The optimisation process identified the value of $p$ directly; however, the mismatch gain $\mathbf{A}_{p}$ is reported in the following sections as it gives a better insight on the difference between $\mathbf{u}_{e}(t)$ and $\mathbf{u}_{\text {sys }}(t)$. This discrepancy has a significant effect in the overall performance, specially when the states get close to the target position.

As observed in Müller et al. [2017], the initial set of parameters used to start the optimisation process had a negligible effect on the resulting optimised parameters, converging consistently to the same set. As a consequence, a common starting point was selected for all conditions and subjects, which is given in Table 1 including the lower and upper limits for each of them.

A pattern-search method was used for all subjects and conditions as implemented in Matlab's Optimisation Toolbox. Pattern-search methods are numerical optimisation routines that avoid the calculation or approximation of derivatives to minimise an objective function [Torczon 1997]. Based on a starting point, the method tries to find a new point that has a lower objective function value. The selection of the points to test is done based on pattern vectors which define points around the initial guess. If a lower objective function value is obtained for a particular point, then the point gets selected and a new set of scaled pattern vectors are applied (increasing the effective search area). If there is no point with a lower objective function value, then the area covered by the pattern vectors is reduced by a predefined factor. The process is repeated until a minimum is found. In general, these methods are useful to speed up repetitive optimisation tasks. 
Table 1. Initial Optimisation Values

\begin{tabular}{ccccccc}
\hline & Parameter & Initial value & Min. value & Max. value & Units \\
\hline \multirow{2}{*}{ LQR } & $\mathbf{Q}_{o}$ & 10 & 0.00001 & 100,000 & - \\
& $\mathrm{Q}_{c, \text { diag }}$ & {$\left[\begin{array}{cccc}1 & 1 & 1 & 1\end{array}\right]$} & 0.00001 & 100,000 & - \\
\hline \multirow{2}{*}{ Timing } & $q$ & 0.03 & 0.001 & 1 & - \\
& $\Delta_{\text {ol }}^{\text {min }}$ & 0.05 & 0.03 & 1 & sec \\
\hline Mismatch & $p$ & 0.9 & 0.1 & 2 & - \\
\hline
\end{tabular}

The values are shown in three different categories: $Q_{c}$ and $Q_{o}$ correspond to the $L Q R$ design parameters, the threshold $q$ and the minimum open-loop interval $\Delta_{o l}^{\min }$ as timing parameters, and finally the value of $p$ which determines the mismatch gain $\mathrm{A}_{p}=1-p$. The minimum and maximum values for each parameter used as limits during the optimisation process are also shown.

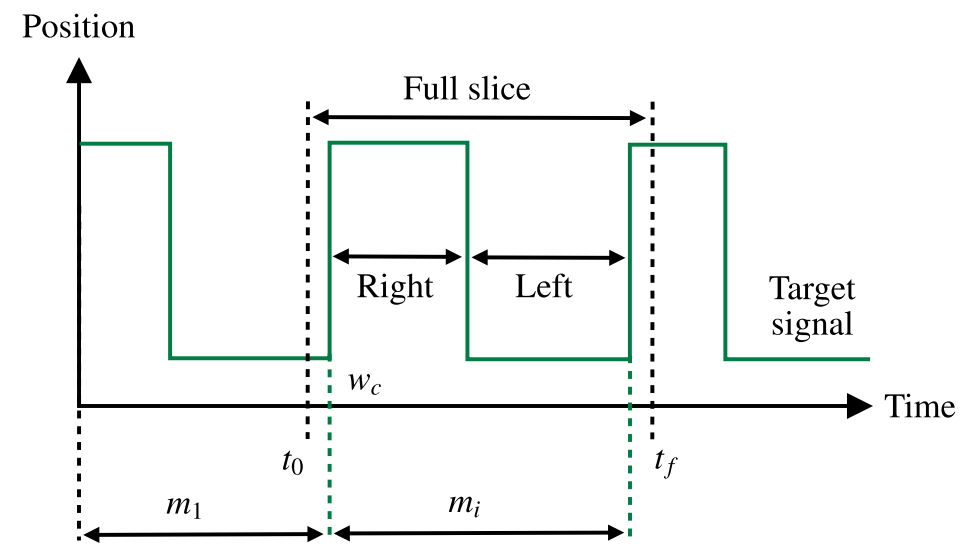

Fig. 4. Data partitioning diagram. The figure shows a hypothetical target signal $w(t)$ (in green) to illustrate how the slices were delimited. The initial point of the slice $t_{0}$ starts 10 samples before a change in reference $w_{c}$ where the participant has to move the pointer position to the target on the right side of the screen. The ending point $t_{f}$ is 10 samples after the next change in reference in the same direction. The second portion of the slice (shown as Left) corresponds to the movement from the target on the right to the target on the left. As described in section 7.3, $m_{1}$ represents the best controller of the bank $m$, which is used to start a simulation and $m_{i}$ is a randomly selected controller from the same bank.

\subsection{Data Partitioning}

The amount of data used for each optimisation differs from Müller et al. [2017]. Instead of fitting an intermittent controller to the entire block of trials, the data were partitioned according to the following criteria: the time elapsed between two successive changes in the target position would constitute a full slice. Essentially, each slice is composed of the pointer trajectories made by the subject to reach consecutive targets (two consecutive trials). An individual IC is then fitted to each slice. In Figure 4, a prototypical target signal is used to illustrate how the data was partitioned into individual slices. Each block had 80 trials, this resulted in 40 full slices per block. The optimisation was carried out using the information of the last 20 slices in the block, leaving the rest for evaluation.

\subsection{Criteria for Model Fitness via Cost Functions}

To establish how well the intermittent controller fits the experimental data, we use a general cost function $J$ measuring the difference between pointer movement predicted by the model and actual pointer movement, which also includes the difference in terms of the pointer velocity. The 
optimiser considers the root mean squared error (RMSE) of these quantities as follows:

$$
J=c_{p} \sqrt{\frac{\sum_{i=1}^{n_{j}}\left(\hat{y}_{i}-y_{i}\right)^{2}}{n_{j}}}+c_{v} \sqrt{\frac{\sum_{i=1}^{n_{j}}\left(\hat{v}_{i}-v_{i}\right)^{2}}{n_{j}}},
$$

where $\hat{y}$ and $\hat{v}$ are the simulated pointer position and velocity, respectively. The number of samples considered for each slice is shown as $n_{j}$ and the constants $c_{p}$ and $c_{v}$ denote values that act as weights for each of the errors. The errors in Equation (37) between the positions and velocities are squared, the result is averaged over the number of samples in each trial and then the square root of the obtained quantity is computed. This formulation provides a clear basis to evaluate the fitness of the resulting intermittent controllers. For the optimisation procedure, the values of $c_{p}$ and $c_{v}$ were set to 0.5, resulting in equal weights for each of the error terms in Equation (37).

\subsection{Controller Switching}

The resulting models of the optimisation procedure can be used to carry out a simulation over a collection of trials for each participant. This can be done by selecting the best model available according to different criteria; for instance, selecting the model with the lowest RMSE score or the one that produced the lowest value of the cost function defined in Equation (37). A simulation using this criterion would yield a response that is acceptable in terms of accuracy and steady-state error; however, it would not be able to capture some of the inherent variability of human control. To create a controller that exhibits an appropriately wide variety of responses, we took a multiplemodel approach, instead of just applying a single model for all trials. This collection of parameters which are compatible with individual realisations of the behaviour helps represent the behavioural variability.

For all participants, a bank of models, which we will call $m$, was generated for each of the conditions in the experiment. This bank is essentially a ranked list based on the value of Equation (37) that contains the intermittent controllers that were derived using the optimised parameters for each slice. This amounts to 20 controllers per condition. To start a simulation using this multiple model approach, the best controller in the bank, or $m_{1}$, is used against the first trial, which is composed of an initial pointer movement towards the right target followed by a second movement to the left target. This is illustrated in Figure 4 , where $w_{c}$ shows the moment in time when the target changes. At $w_{c}$, a new controller $m_{i}$ is selected from the model bank and applied for the duration of the next trial only, where $i$ represents a random integer number between 1 and 20 . This random selection of controllers from the bank $m$ starts only after the first trial has finished and it is maintained until all the trials are completed. This effectively ensures that different controllers are used for a pair of back and forth movements, producing a trial response that is slightly different compared to the previous trial, resulting in a more variable trajectory overall.

The following section introduces the results obtained when this multiple-model approach is used to generate dynamic responses in order to compare them to the experimental data.

\section{MODELLING RESULTS}

The results obtained from the optimisation process applied to all participants and conditions are presented in this section. First, a visualisation of the observed variability in some of the responses is introduced, then the optimisation parameters obtained in each condition are shown including some of the dynamic responses showing the pointer position evolution, phase planes where position and velocity are compared, and Hooke plots which show the acceleration profiles through time. 


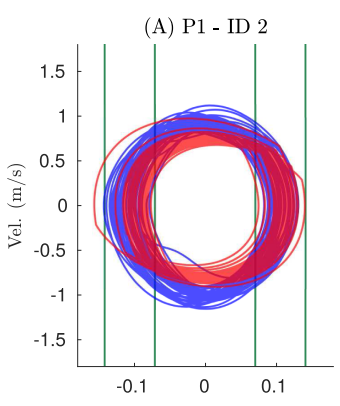

(D) P1 - ID 8

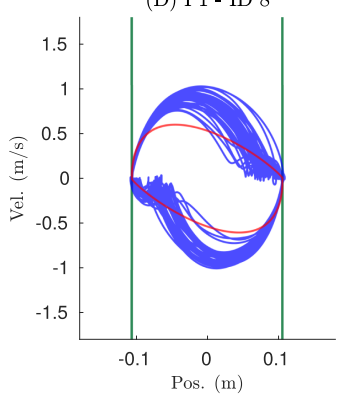

(B) P2 - ID 2

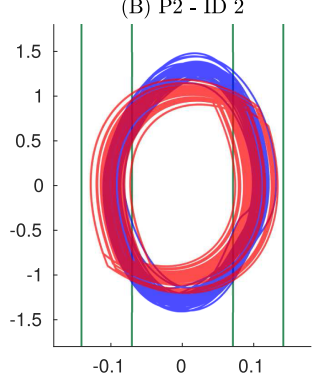

(E) P2 - ID 8

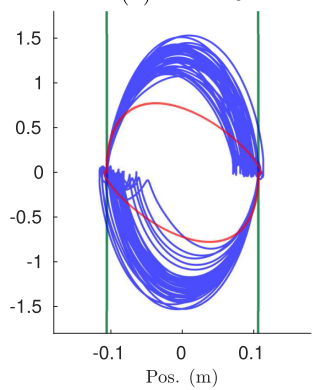

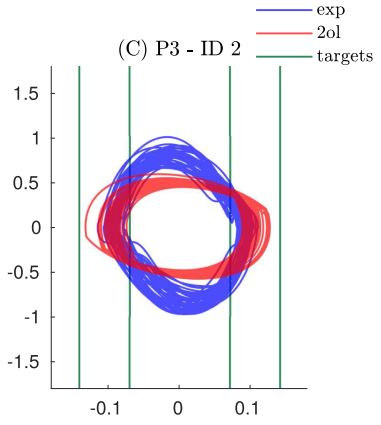

(F) P3 - ID 8

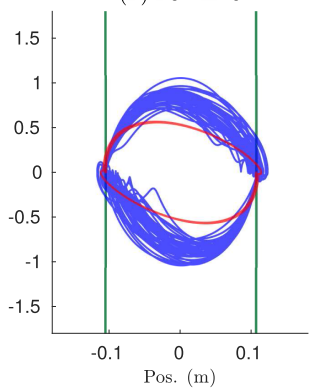

Fig. 5. Phase planes showing pointer position vs velocity for participants 1, 2, and 3. Experimental trajectories for each participant are shown in blue, left and right target limits are represented by green vertical lines, and the trajectories in red correspond to the 2ol model from Müller et al. [2017]. A-C show data for a distance of $212 \mathrm{~mm}$ with a target width of $70.6 \mathrm{~mm}$, corresponding to an ID of 2. D-F show data for the same distance between targets but with a different target width of $0.83 \mathrm{~mm}$ (ID 8). Participants 1, 2, and 3 are represented by each of the columns from left to right. From the simulated trajectories in red, it is clear that the $2 \mathrm{ol}$ model provides some degree of variability for ID 2 but when the ID increases, it does not reproduce the observed variability of the experimental trajectories.

\subsection{Response Variability}

The ID of the task has a significant impact on the overall behaviour. In Figure 5, the phase planes (pointer velocity in the vertical axis vs pointer position in the horizontal axis) for participants 1,2, and 3 are shown to illustrate how the ID influences the control strategy and the human response. The bottom row (D)-(F) shows the experimental data for an ID of 8 , which is regarded as the most difficult task in the experiment. A clear indicator of this is how small the target width is $(0.83$ $\mathrm{mm}$ ), compared to a target width of $70.6 \mathrm{~mm}$ (ID of 2) presented in A, B, and C. The target width is delimited by vertical lines on each side. For each condition, the experimental phase planes are shown in blue, whereas the ones in red correspond to the continuous 20 controller described in Müller et al. [2017]. The 2ol controller is different in many levels when compared to IC, but its most fundamental difference is the fact that it uses feedback at all times to generate the control input that is applied to the system.

The bottom row in Figure 5(D)-(F) shows responses where an initial burst in speed and position is made, commonly known as the surge, followed by a series of small position corrections as the pointer approaches the target. It is clear, for instance, that the landing position for the surge movement differs in every trial-a consequence of human variability. The top row of figures (A)(C) exhibits a drastically different behaviour since the target width is greater, there is variability present but the participants seem to engage in an almost constant motion towards the targets without making corrections as they approach. The 2 ol controller does a relatively good job tracking 

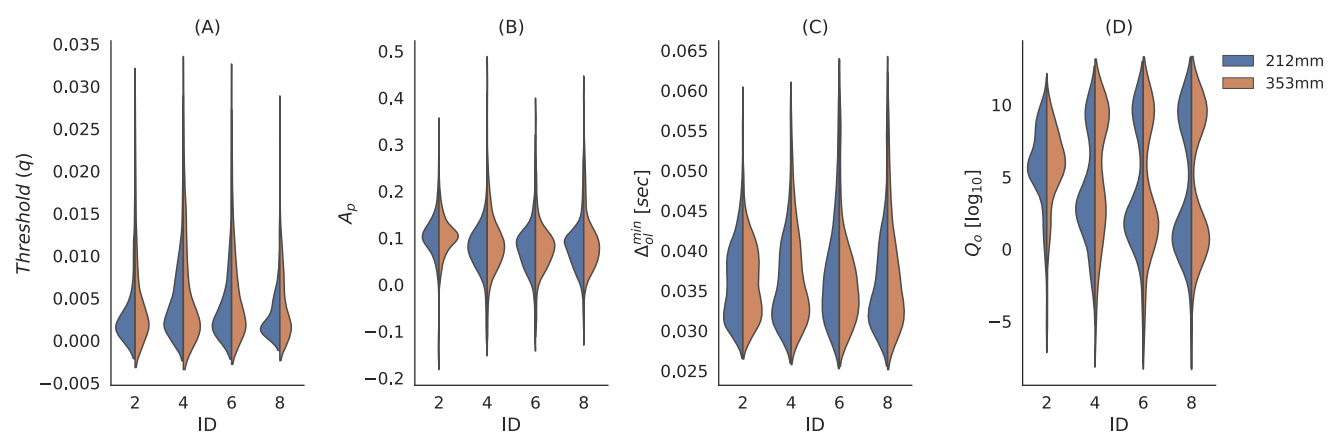

Fig. 6. Optimised controller parameters for each condition. (A) the threshold $q$, (B) mismatch gain $\mathrm{A}_{p}$, (C) the minimum open-loop interval $\Delta_{o l}^{\mathrm{min}}$, and (D) the observer gain $\mathrm{Q}_{o}$ are shown as violin plots including data of all subjects and categorised by ID (horizontal axis). The results are also grouped according to the two values of distance between targets used in the experiment (left: $212 \mathrm{~mm}$ and right: $353 \mathrm{~mm}$ ). The shape of the distributions for ID 2 is slightly different compared to the rest of the conditions.

the easy conditions (ID 2); however; when applied to the most difficult condition (ID 8), the phase plane does not show the same level of variability seen in the experimental data. The parameters of the 2ol controller were also obtained via optimisation as described in Müller et al. [2017] resulting in an individual parameter set for each condition, which was subsequently applied in simulation.

\subsection{Optimised Parameters and Their Interpretation}

The optimisation process had four free scalar parameters $\mathbf{Q}_{o}, \mathrm{~A}_{p}, q$, and $\Delta_{o l}^{\min }$, as well as the matrix $\mathrm{Q}_{c}$, where only its diagonal elements are of interest (the off diagonal entries are zero), thus adding four extra parameters for a total of eight. In Figure 6, the optimised parameters for all subjects are shown when grouped by ID. This includes the threshold $q(\mathrm{~A})$, the gain $\mathrm{A}_{p}(\mathrm{~B})$, the minimum openloop interval $\Delta_{o l}^{\min }(\mathrm{C})$ and finally the observer gain $\mathrm{Q}_{o}(\mathrm{D}$, shown in log scale). For each parameter, the data distribution is shown as a violin plot for the two different distances of $212 \mathrm{~mm}$ (left side) and $353 \mathrm{~mm}$ (right side), respectively, and grouped according to the ID.

Figure 6(A) shows that the threshold $q$ has slightly larger values for ID 2, which is consistent for the distributions of both distances. The data are more concentrated for the cases of ID 4, 6, and 8 as shown by the narrow distributions. All conditions exhibit long tails, indicating the presence of higher thresholds in some of the optimised models. The mismatch gain $A_{p}$ in Figure 6(B) also exhibits a clear trend. For ID 4, 6, and 8, the data distribution is wider for these three cases, indicating that there was less of a mismatch compared to ID2, which shows most of its values around $A_{p}=0.1$. The minimum open-loop interval $\Delta_{o l}^{\min }$ in Figure $6(C)$ is comparable for the different levels of ID, showing that most of the data lie within a 0.03 to $0.05 \mathrm{sec}$ range.

The values for the design matrix $Q_{c}$ are presented in Figure 7 using a log scale, where 7(A)-(D) show the data of the four elements in the diagonal of matrix $Q_{c}$. The first and second elements of $\mathbf{Q}_{c}$ shown in Figure 7(A) and (B) are of particular importance: $\mathbf{Q}_{c 1}$, since it is associated to the position state which is the defined output of the controller, and $Q_{c 2}$ is related to the pointer velocity state. These two values have a distinct impact in the overall transient behaviour. $\mathrm{Q}_{c 1}$ shows higher values across all conditions compared to the other elements in $Q_{c}$, which means that the controller is trying to put more emphasis on the position state in order to reduce the error with respect to the reference. However, comparing the distributions for each ID in Figure 7(A), it can be seen that the optimisation yields controllers with higher values of $Q_{c 1}$ for ID 2 in general, whereas the rest of the IDs have distributions that are similar in shape. This seems to be related to the result shown 
(A)

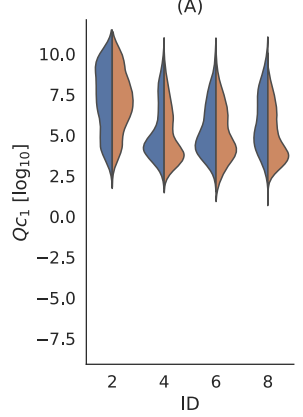

(B)

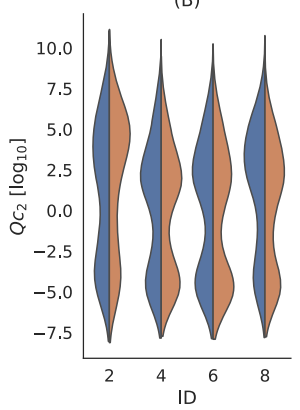

(C)

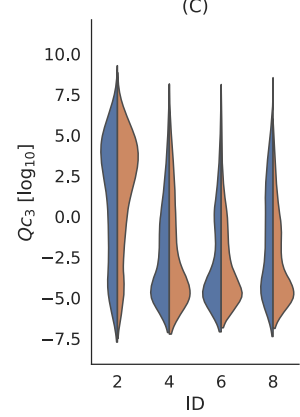

(D)

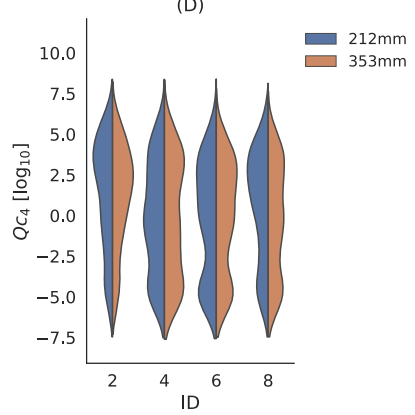

Fig. 7. Optimised $Q_{c}$ parameters for each condition. This figure shows the values of the four elements in the diagonal of matrix $Q_{c}$, for all subjects and segmented by ID (horizontal axis). The results for $\mathbf{Q}_{c 1}, \mathbf{Q}_{c 2}$, $\mathrm{Q}_{c 3}$, and $\mathrm{Q}_{c 4}$ are shown in $\mathrm{A}, \mathrm{B}, \mathrm{C}$, and $\mathrm{D}$, respectively. The violin plots show the data for the two distances between targets (left, in blue: $212 \mathrm{~mm}$ and right, in brown: $353 \mathrm{~mm}$ ). $Q_{c 1}$ has consistently higher values compared to the rest of the elements in $Q_{c}$ and ID 2 has a different distribution shape than the rest of the conditions.

in Figure 6(B) where ID 2 exhibits a larger model mismatch $A_{p}$. A plausible explanation is that the LQR method tries to compensate for the mismatch by assigning a higher weight on the position state, which in the end results in a higher controller gain. For $Q_{c 2}$ in Figure 7(B), the distributions are bimodal for all ID showing lower values in general compared to $Q_{c 1}$. Similarly, the distribution of ID 2 presents more data grouped towards the positive range of values, indicating that the optimisation procedure penalised the velocity state more heavily. This is also in agreement with the result for $\mathrm{A}_{p}$ in Figure 6(B). Overall, the difference in behaviour of ID 2 against the rest is evident, and the higher weights for $Q_{c 1}$ and $Q_{c 2}$ reveal that as the ID increases, a higher degree of precision is needed and therefore the controller becomes more cautious by producing smaller gains $\mathbf{k}$.

As a result of the LQR method that involves the design matrix $\mathbf{Q}_{c}$, as shown in Equation (35), a set of controller gains $\mathbf{k}$ can be obtained to implement the control law described in Equation (5). Figure 8 presents the values of each element in $\mathbf{k}$ in a log scale, grouped by ID, and displaying side by side the distribution for each target distance in the experiment. It can be seen, by comparison with Figure 7, that ID 2 still shows higher values for all the elements in $\mathbf{k}$. However, the bimodal distributions of $Q_{c 2}$ in Figure 7(B) were converted to a single low peak with long tails towards the higher values of $\mathbf{k}$.

\subsection{Dynamic Responses}

The position and velocity RMSE is calculated to give a measure of performance for the optimised controller in each condition according to Equation (37). The results across all participants are shown in Figure 9. The left figure is the position RMSE for both distances (212 and $353 \mathrm{~mm}$ ) and the one on the right side corresponds to the velocity RMSE, with the data being grouped according to the ID. In both cases, the error when the distance between left and right targets is $353 \mathrm{~mm}$, is larger compared to the errors registered for $212 \mathrm{~mm}$. The position error is larger in ID 2 for both distances compared to the rest of the conditions, suggesting that the more highly variable behaviour in low constraint cases is harder to model with this particular model. In terms of velocity error, when the task is difficult (i.e., ID 8), the values are smaller compared to the rest of the conditions. This suggests that since more precision is needed due to the target width being reduced, a more cautious regulation is required by the user, in terms of velocity. A similar argument can be made for the position error. In general, the easier conditions (ID 2 in particular) allow a greater degree of error because the targets are wider. 

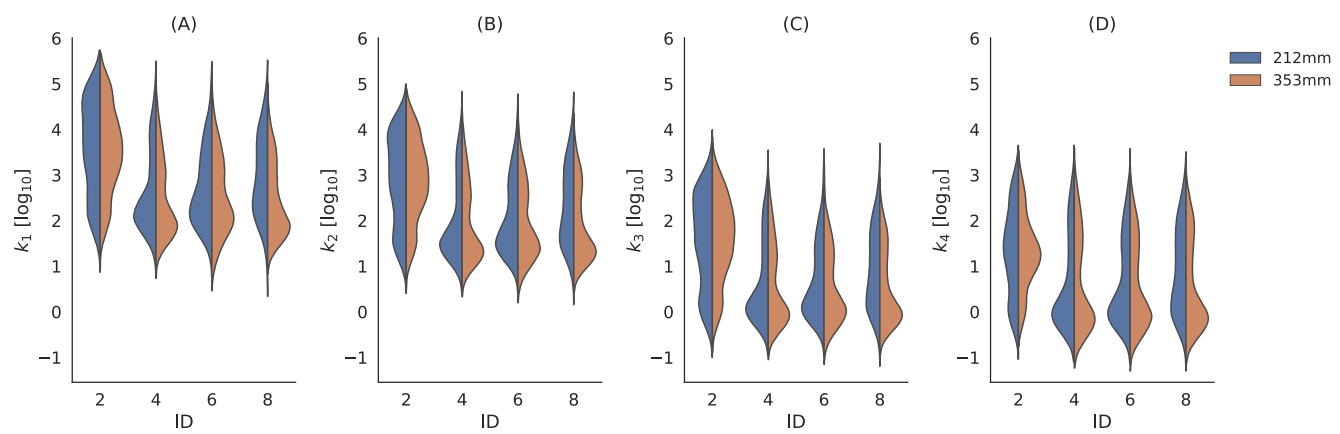

Fig. 8. Resulting controller gains $\mathbf{k}$ for each condition. This figure shows the four elements of the gain vector $\mathbf{k}$ for all participants and segmented by ID (horizontal axis). The results for $\mathbf{k}_{1}, \mathbf{k}_{2}, \mathbf{k}_{3}$, and $\mathbf{k}_{4}$ are shown in A, $\mathrm{B}, \mathrm{C}$, and D, respectively. Each violin plot presents the results for the two distances between targets (left, in blue: $212 \mathrm{~mm}$ and right, in brown: $353 \mathrm{~mm}$ ) side by side. The controller gains follow a similar trend compared to the design matrix $Q_{c}$ from Figure 7, with ID 2 showing some differences in shape against the rest of the conditions.
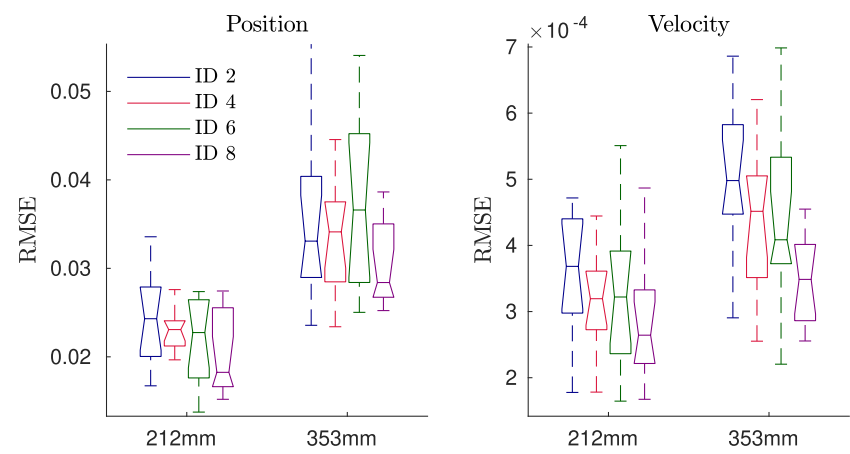

Fig. 9. RMSE comparison for the position and the velocity profiles. The left and right figures show position and velocity RMSE, respectively. The results in both cases are grouped according to the distance between left and right targets $(212 \mathrm{~mm}$ and $353 \mathrm{~mm}$, horizontal axis) and also according to the ID of the task, where each box plot represents one of the four conditions of the experiment.

The following figures show the time-series, phase planes, and Hooke plots for participant 10, as well as some comparisons made for all participants. Figure 10 shows the phase planes for ID 8 when the distance between the left and right targets is the shortest $(212 \mathrm{~mm})$. This is the most difficult task since the targets are not only close to each other but the target width is also small $(0.83 \mathrm{~mm})$. The human response (in blue) tends to fall short of the target (vertical lines in green) and subsequently approaches it via a series of small corrections, as well as overshooting it. The controller switching strategy described in 7.3 yields trajectories that capture the variability in some regions of the phase planes and it is particularly good at reproducing the trajectories that overshoot the target. This can be seen more evidently in participants 3, 4, 8, 10, and 12. In Figure 10(H), the trajectories that start from the left target depart from small area to then approach the right target, widening and spreading as they get closer.

In Figure 11, the phase planes for a distance of $212 \mathrm{~mm}$ are shown when the ID is 2 (target width is the largest). The response of the controller is capable of capturing the overall behaviour and some of its associated variability, as the shape of the response changes from a sequence of corrections (Figure 10) to a more continuous circular movement. In this case, since the difficulty 
(A) - P1

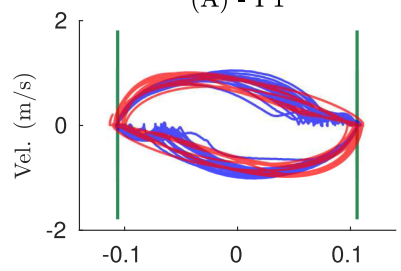

(D) - P4

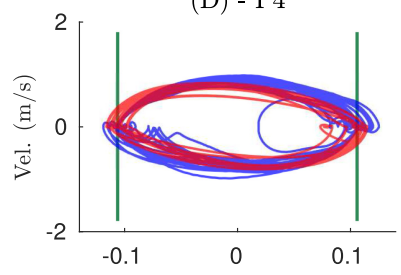

(G) - P7

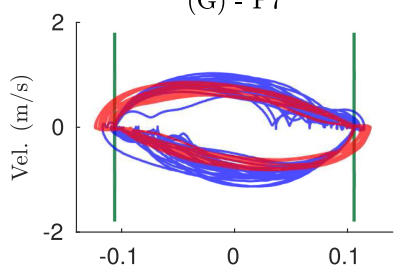

(J) - P10

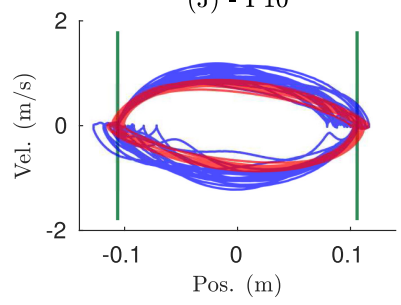

(B) - P2

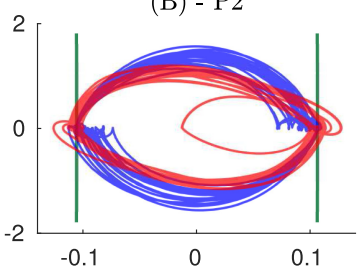

(E) - P5

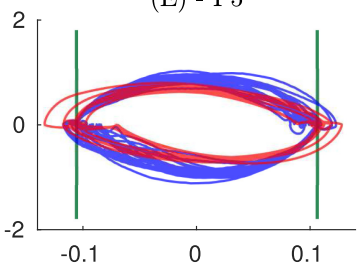

(H) - P8

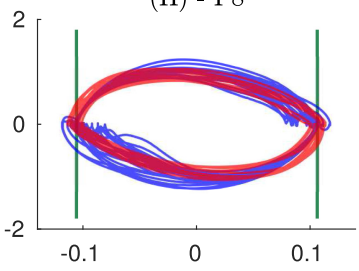

(K) - P11

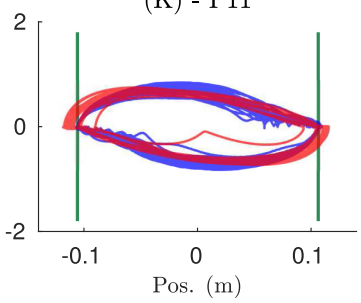

(C) - P3

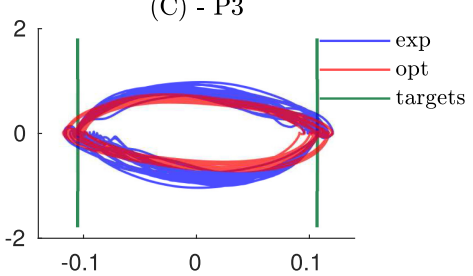

(F) - P6

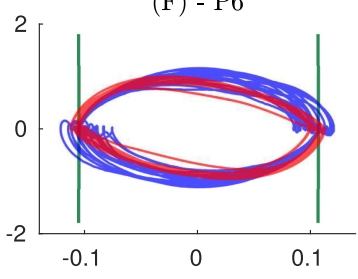

(I) - P9

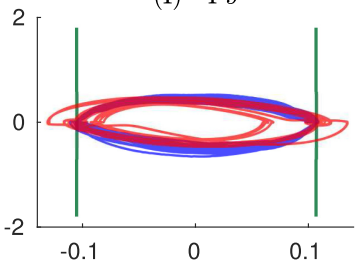

(L) - P12

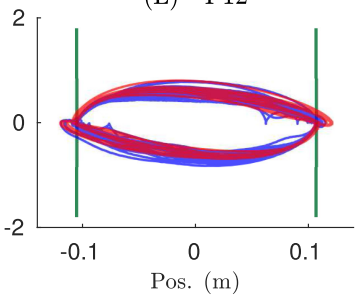

Fig. 10. Phase planes for all participants, a distance between targets of $212 \mathrm{~mm}$ and an ID of 8 . The phase planes compare the pointer position (horizontal axis) against the pointer velocity (vertical axis). The trajectories in blue correspond to the experimental data and the ones in red represent the resulting trajectories when the optimised IC is used. The location of the targets is shown by the vertical lines in green. The optimised IC trajectories capture more of the observed variability when compared with the 201 model responses shown in Figure 5 for this condition.

is low, the participants can afford a greater degree of error in the initial portion of the movement leading to trajectories with greater variability and with no correction as they land in the target area. The controller, in most cases, exhibits trajectories that follow closely the ones generated by each participant. ID 2 is the condition that is substantially different in terms of behaviour than all the rest, as described in Figure 7(A) and (B).

In Figure 12, the time-series associated with the pointer position of participant 10 is shown for the initial part of each trial, where the position recorded from the experiment is in blue, the trajectory generated by the IC is in red, and the target signal (reference) is shown in green. Also, the moments in time when the IC generated an event to trigger the use of feedback are represented by vertical lines in gray. 

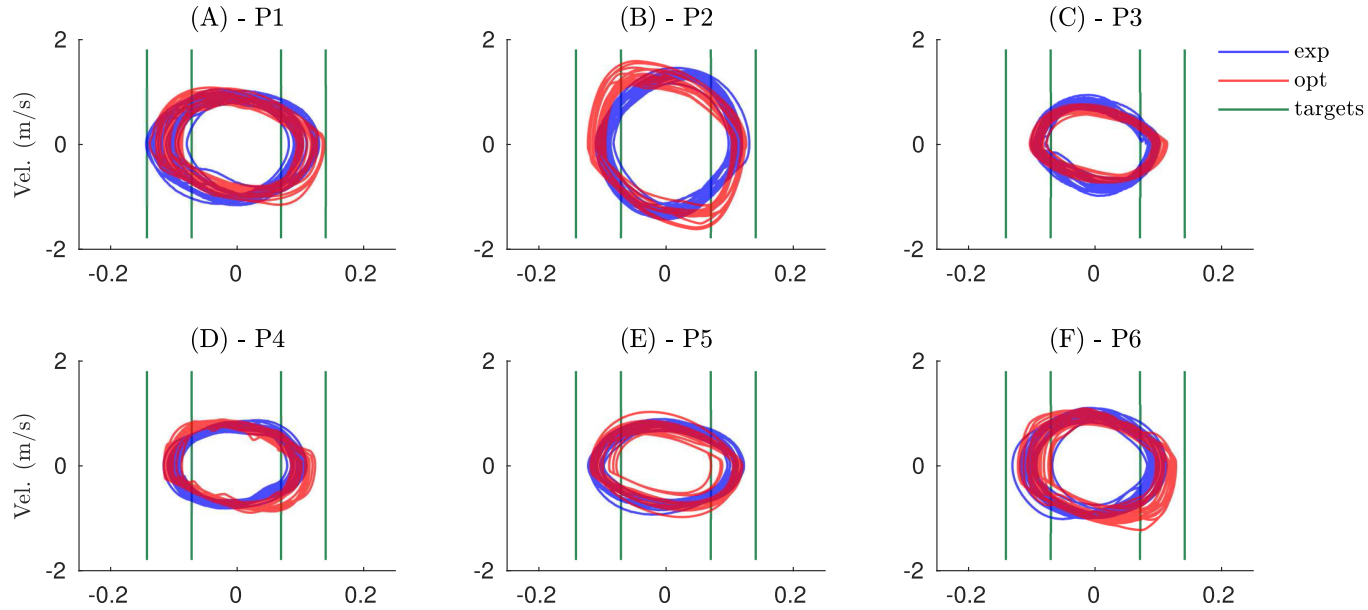

(E) - P5

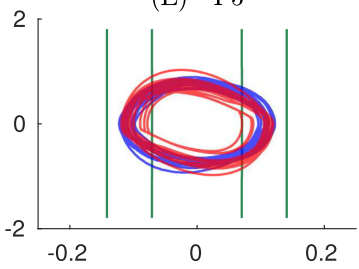

(F) - P6

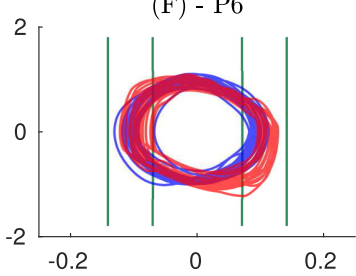

(G) - P7

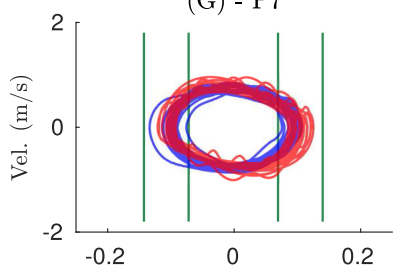

(H) - P8

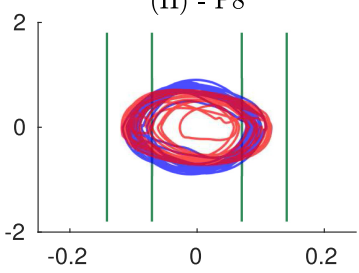

(I) - P9
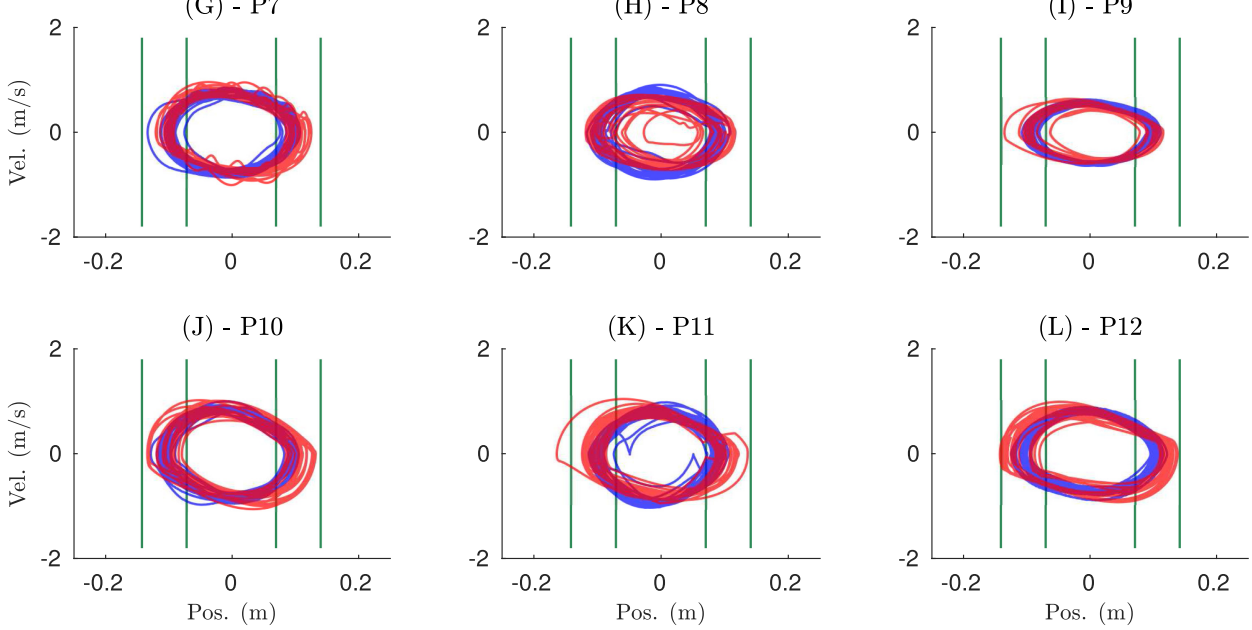

Fig. 11. Phase planes for all participants, a distance between targets of $212 \mathrm{~mm}$ and an ID of 2. The phase planes compare the pointer position (horizontal axis) against the pointer velocity (vertical axis). The trajectories in blue correspond to the experimental data and the ones in red represent the resulting trajectories when the optimised IC is used. The location of the targets is shown by the vertical lines in green. The optimised phase planes in red follow the shape of the experimental ones, displaying the variability of the trajectory.

The left column in Figure 12 shows data for a distance between left and right targets of 212 $\mathrm{mm}(\mathrm{A}, \mathrm{C}, \mathrm{E}, \mathrm{G})$, and the right column shows it for $353 \mathrm{~mm}(\mathrm{~B}, \mathrm{D}, \mathrm{F}, \mathrm{H})$. Each row in the figure corresponds to a different ID, starting from 2 at the top and ending with 8 at the bottom. It is possible to see how in all conditions, both the experimental and IC trajectories follow the reference closely. In particular, the trajectory generated by the IC exhibits a small degree of error compared to the experimental result, especially as the response approaches the target. Also, for the most difficult conditions, such as ID 6 or 8 (E, F, G, and H), the position follows a more traditional step response with a small steady-state error. However, the top row shows how the response becomes more similar to a sinusoidal signal due to the quick changes in the target signal in green.

IC generates events when the comparison between the hold and the observed states exceeds the specified threshold value as shown in Equation (26). Due to the controller switching strategy 
(A) $212 \mathrm{~mm}$ - ID 2

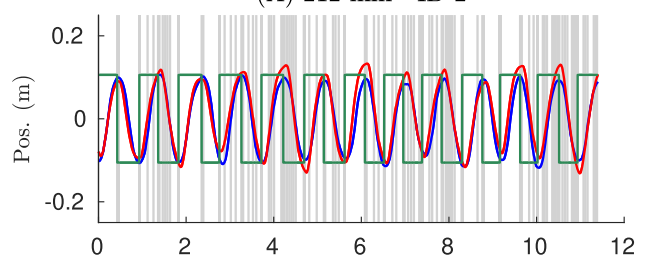

(C) $212 \mathrm{~mm}$ - ID 4

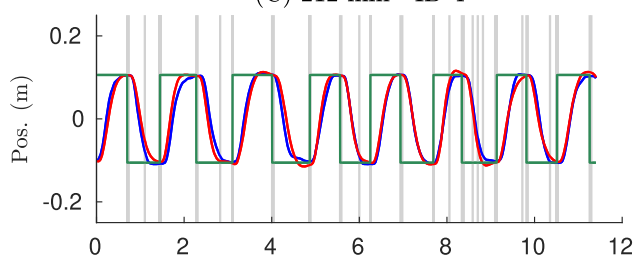

(E) $212 \mathrm{~mm}$ - ID 6

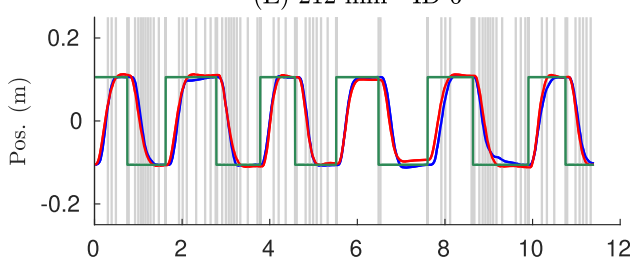

(G) $212 \mathrm{~mm}$ - ID 8

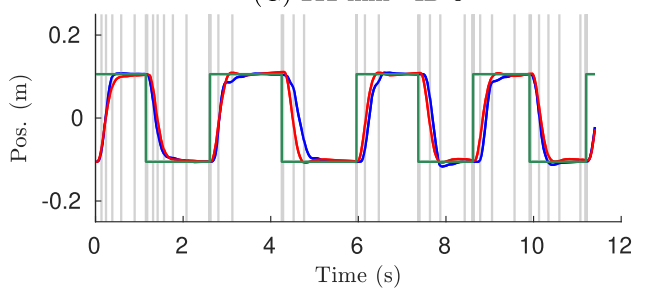

(B) $353 \mathrm{~mm}$ - ID 2

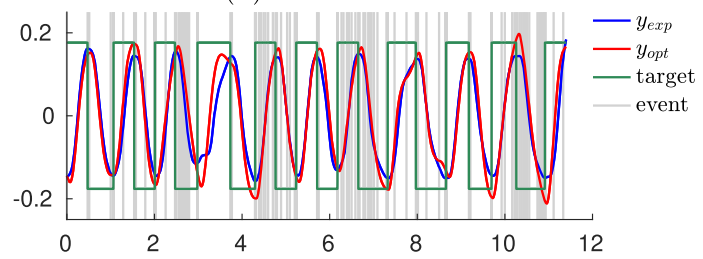

(D) $353 \mathrm{~mm}$ - ID 4

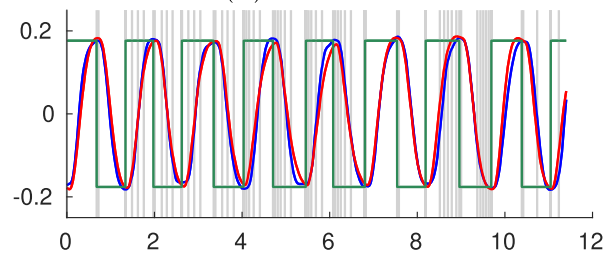

(F) $353 \mathrm{~mm}$ - ID 6

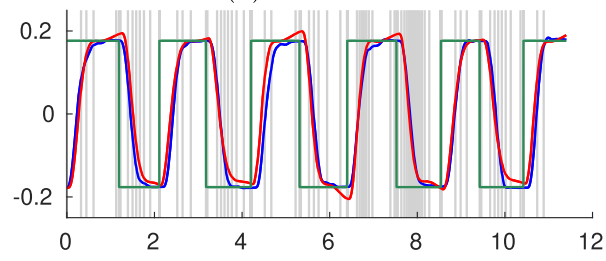

(H) $353 \mathrm{~mm}$ - ID 8

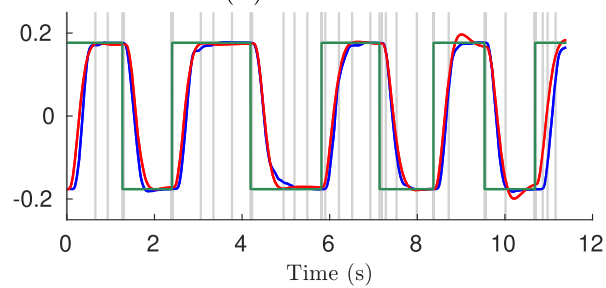

Fig. 12. Pointer position for participant 10 in all conditions. Each row corresponds to a particular ID, starting with 2 for the top row and ending with 8 at the bottom. The column on the left shows the pointer position (vertical axis) for a distance between targets of $212 \mathrm{~mm}(\mathrm{~A}, \mathrm{C}, \mathrm{E}, \mathrm{G})$. The right column shows the same quantities for a distance of $353 \mathrm{~mm}(\mathrm{~B}, \mathrm{D}, \mathrm{F}, \mathrm{H})$. The horizontal axis shows around 11 seconds of each condition. The participant response $y_{\text {exp }}$ is shown in blue, while the trajectories generated by the IC are shown in red as $y_{o p t}$. The target or reference signal is shown in green. The vertical lines in grey show the instances in time when an event was triggered by the optimised IC, signalling the use of feedback. The simulated IC trajectories follow closely the ones generated by the participant; for each change in reference, subtle differences can be seen in the IC trajectories as a consequence of the controller switching approach explained in Section 7.3.

described in Section 7.3, a different threshold is applied at every change in reference $w_{c}$ (Figure 4), which creates different trigger patterns depending on the trial and the model that was selected. This is evident in Figure 12(B) and (G) where the trials around 8 secs exhibit events that are close in temporal proximity; however, when the next trial starts, the open-loop intervals $\Delta_{o l}$ become larger due to the different parameters being applied including a new threshold $q$ value, which results in a slower triggering rate compared to the previous trial. During the time between events, the IC is evolving in an open-loop configuration, using only states that are generated internally by Equation (14). 

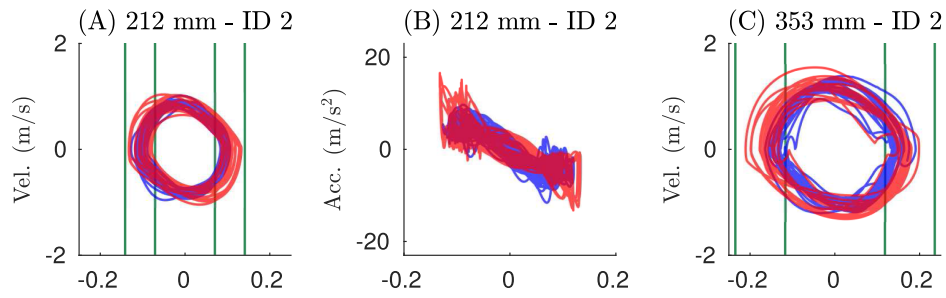

(D) $353 \mathrm{~mm}$ - ID 2

(E) $212 \mathrm{~mm}$ - ID 4

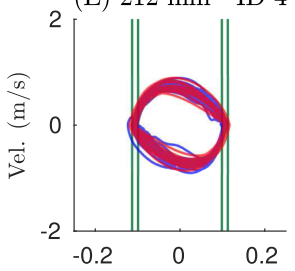

(F) $212 \mathrm{~mm}$ - ID 4
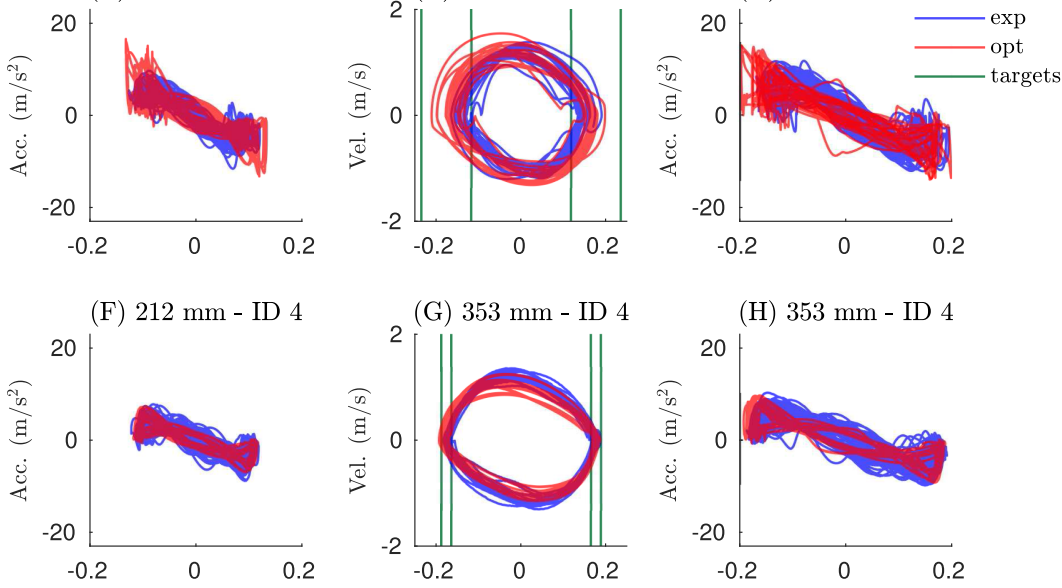

(H) $353 \mathrm{~mm}$ - ID 4

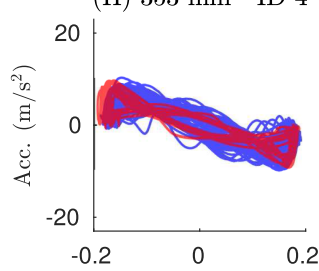

(I) $212 \mathrm{~mm}$ - ID 6

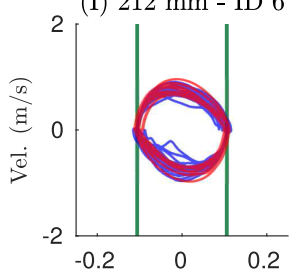

(J) $212 \mathrm{~mm}$ - ID 6
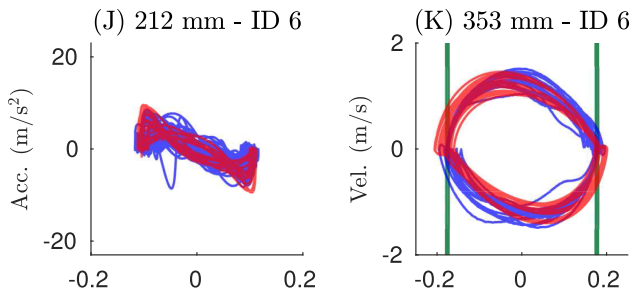

(L) $353 \mathrm{~mm}$ - ID 6
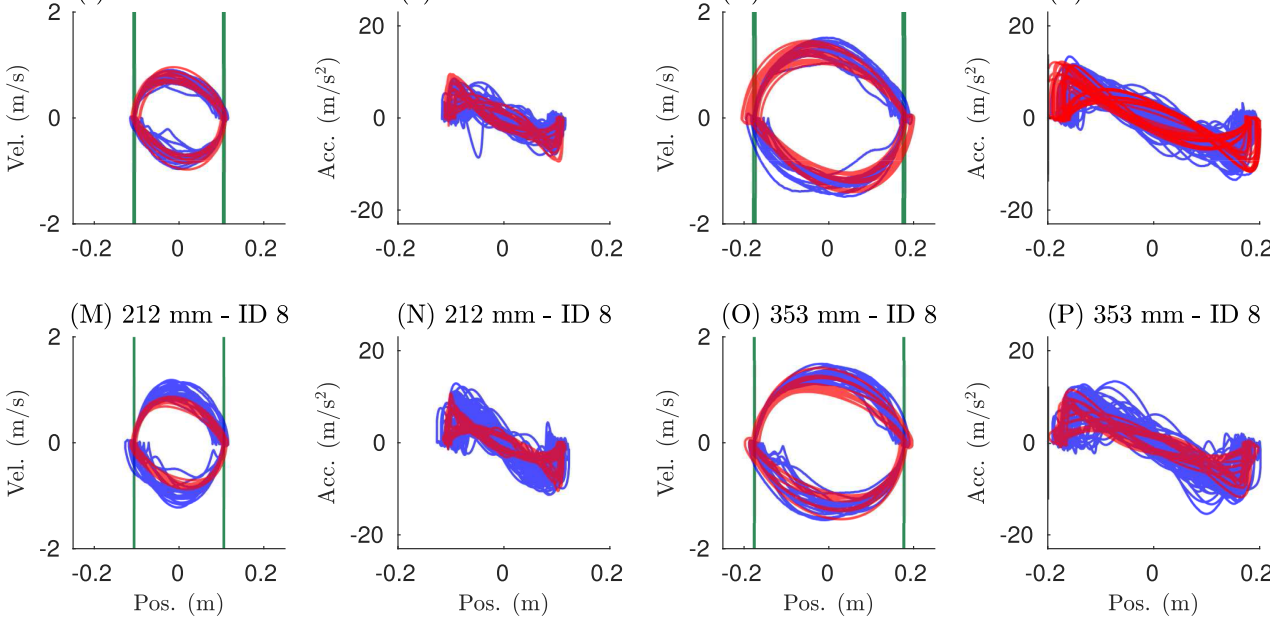

(P) $353 \mathrm{~mm}$ - ID 8

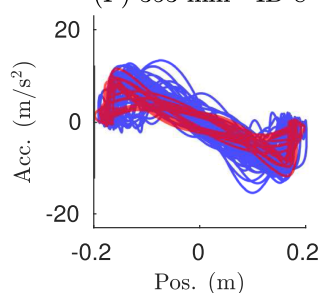

Fig. 13. Phase planes and Hooke plots for participant 10 in all conditions. Each row corresponds to a particular ID, starting with 2 for the top row and ending with 8 at the bottom. The two columns on the left show the phase planes (pointer position vs velocity) and Hooke plots (pointer position vs acceleration) for a distance between targets of $212 \mathrm{~mm}(\mathrm{~A}, \mathrm{~B}, \mathrm{E}, \mathrm{F}, \mathrm{I}, \mathrm{J}, \mathrm{M}, \mathrm{N})$. The two columns on the right show the same quantities for a distance of $353 \mathrm{~mm}(\mathrm{C}, \mathrm{D}, \mathrm{G}, \mathrm{H}, \mathrm{K}, \mathrm{L}, \mathrm{O}, \mathrm{P})$. The participant's response is shown in blue, while the trajectories generated by the IC are shown in red. The target or reference signal is shown in green using vertical lines.

Figure 13 presents the phase planes and Hooke plots of participant 10 for all conditions. The first two columns from left to right contain data for a distance between targets of 212, whereas the third and fourth columns represent a distance of $353 \mathrm{~mm}$. Each row corresponds to a specific ID, starting from ID 2 in the top row and ending with ID 8 at the bottom. In general, the phase planes follow the overall behaviour of the human response (in blue); however, it is possible to see that the IC response (in red) captures the variability seen in the experimental result in all conditions, being slightly less accurate as the difficulty increases.

The trajectories in Figure 13(E) and (O), provide some insight into the type of control applied by the participants in these conditions. Once the trajectory approaches the target, the control policy 
that is used results in a reduction of the pointer velocity accompanied by a sequence of small corrections to reduce the error (more frequent in difficult conditions), or in an error in the opposite direction in the form of overshoot (when the response passes the intended target). For these two cases, the IC output not only covers almost the entire range of possible trajectories generated by this participant, but also reproduces the overshoot behaviour that was previously mentioned. The Hooke plots (second and fourth columns) compare how the pointer acceleration changes with time. In Figure 13(F) and (P), the acceleration trajectory generated by the IC is smooth (in red), and follows the experimental result (blue) in terms of its overall shape.

\subsection{Open-Loop Intervals}

The histograms of the open-loop intervals $\Delta_{o l}$, generated by the intermittent controller, is presented in Figure 14, where all the $\Delta_{o l}$ values for a specific condition were considered for all the participants in the experiment. These values are the actual open-loop intervals recorded from simulation, which can be greater than the minimum open-loop interval $\Delta_{o l}^{\min }$ for a particular slice. The minimum open-loop interval $\Delta_{o l}^{\min }$ belongs to the set of parameters used in the optimisation approach described in Section 8.2, with distributions shown in Figure 6. In Figure 14, we decided to overlap both $\Delta_{o l}$ and $\Delta_{o l}^{\min }$ to show the differences between the two and their relationship with specific conditions of the experiment.

Figure 14(A), (C), (E), and $(\mathrm{G})$ represents the trials where the distance between targets is 212 mm. Similarly, Figure 14(B), (D), (F), and (H) corresponds to $353 \mathrm{~mm}$. From top to bottom, each ID is shown as a row, starting with ID 2 at the top (A, B) and ending in ID 8. The values of $\Delta_{o l}$ were encapsulated as a histogram (in grey) which shows the frequency of a particular value for a single condition or ID, and its scale is shown on the left $y$ axis of each subplot. The values of $\Delta_{o l}^{\min }$ are shown as either blue or brown histograms, corresponding to distances between targets of $212 \mathrm{~mm}$ and $353 \mathrm{~mm}$, respectively.

The results in Figure 14 show that the majority of the intervals are short in general, having higher frequencies at lower values of $\Delta_{o l}$. Considering Figure 14(A) and (B), it is clear that histograms in grey decay at a faster rate compared to the rest of the conditions for both distances, showing very few instances of $\Delta_{o l}$ beyond $0.25 \mathrm{sec}$. For ID 4, 6, and 8, the tail of the histogram extends beyond the $0.25 \mathrm{sec}$ consistently, meaning that the open-loop intervals are longer in general compared to the easiest condition of ID 2.

The small values of $\Delta_{o l}$ for ID 2 seem to be caused by the effects of the identified threshold $q$ and the mismatch gain $\mathrm{A}_{p}$ (shown in Figure 6) and the fact that the IC model had more trouble fitting the response for this particular condition in terms of the target position and velocity, which is displayed in Figure 9. The RMSE for ID 2 in both distances is larger that than the corresponding error in the other conditions, probably as a result of the model trying to capture the variability observed in these trials; however, the poor fit results in low threshold $q$ values which indicates that prediction errors would be addressed by using feedback more often. If the threshold $q$ is low, the triggering patterns of IC would most likely become close to the imposed minimum open-loop interval $\Delta_{o l}^{\mathrm{min}}$, and in some cases, triggering would be as fast as the minimum open-loop interval for that particular model would allow.

This behaviour is also affected by the mismatch gain $A_{p}$. Having large values of $A_{p}$ means that the control input that is applied to the system is different from the input that would generate the desired performance. Since the input is affected, the states of the system might not reach the specified targets and this would eventually lead to higher triggering rates.

The minimum open-loop intervals $\Delta_{o l}^{\mathrm{min}}$, for both distances, have similar histogram distributions that extend to $0.05 \mathrm{sec}$ approximately. However, the most difficult conditions, i.e., ID 6 and 8, seem 

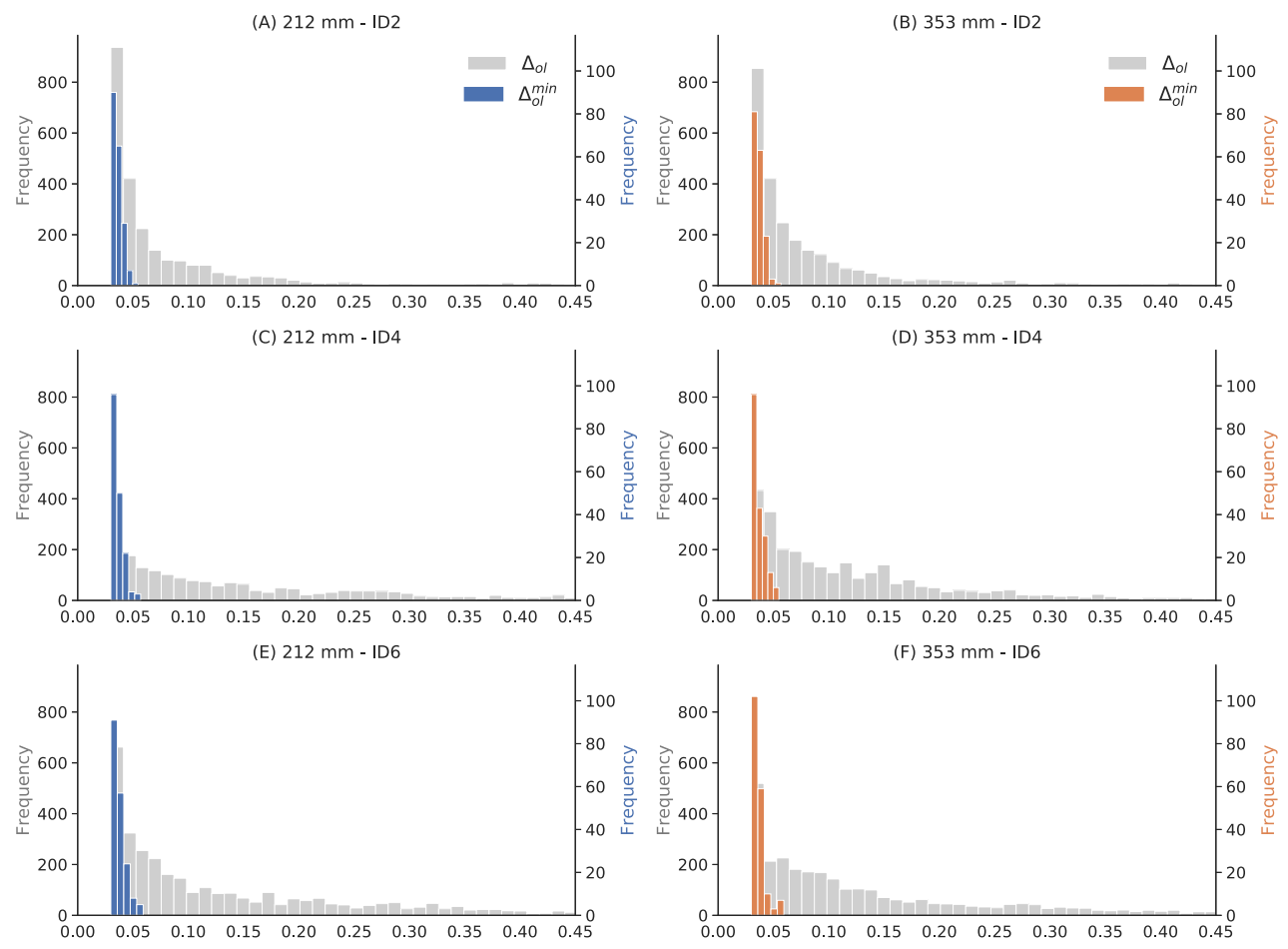

(G) $212 \mathrm{~mm}$ - ID8

(H) $353 \mathrm{~mm}$ - ID8
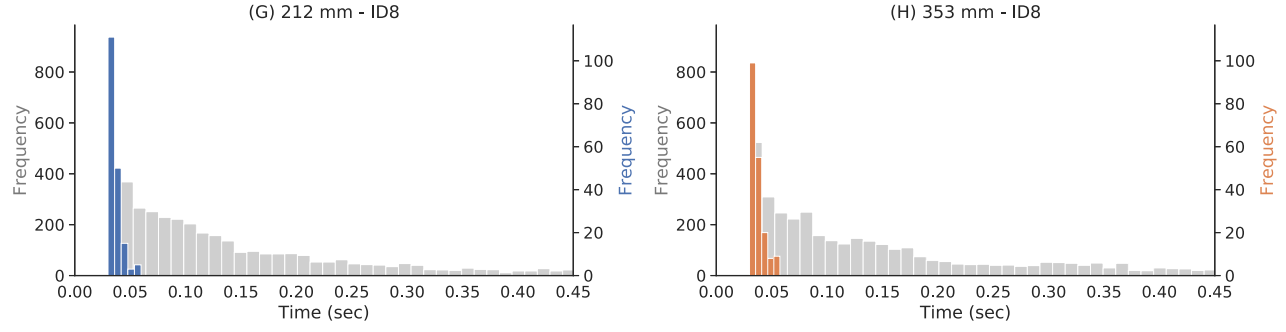

Fig. 14. Open-loop interval distributions for all participants. The actual open-loop interval $\Delta_{\text {ol }}$, generated by IC, is shown as a grey histogram summarising the data of all participants for a particular condition. Similarly, blue and brown histograms corresponding to the minimum open-loop interval $\Delta_{o l}^{\mathrm{min}}$ are shown for a distance between targets of $212 \mathrm{~mm}$ and $353 \mathrm{~mm}$, respectively. The vertical axis corresponds to the frequency or bin count of a particular value of $\Delta_{o l}$ (left axis) and $\Delta_{o l}^{\text {min }}$ (right axis). The horizontal axis displays the time in seconds.

to have slightly more values of $\Delta_{o l}^{\min }$ around 0.05 than the rest of the conditions. Figure 14 provides evidence of the interplay between the threshold $q$ and $\Delta_{o l}^{\mathrm{min}}$ as well as for the event-driven nature of IC, since even when IC could trigger as fast as $\Delta_{o l}^{\min }$ all the time (blue and brown histograms), it does it only after the threshold has been exceeded, leading to the longer open-loop intervals shown by the grey histograms.

\section{DENSITIES BASED ON REPEATED SIMULATIONS}

Using the controller switching strategy from Section 7.3, multiple simulations were carried out using the optimised parameters and the associated models in order to create a probability distribution of the IC simulation results, in the phase space. The most basic non-parametric approach 

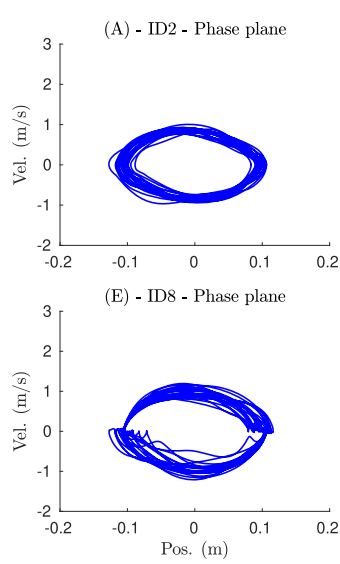

(B) - ID2 - 2D Histogram

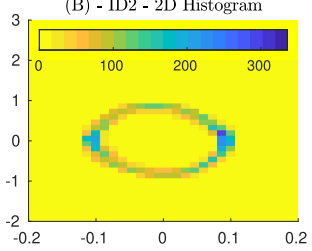

(F) - ID8 - 2D Histogram

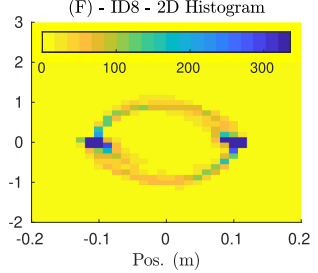

(C) - ID2 - 3D Histogram

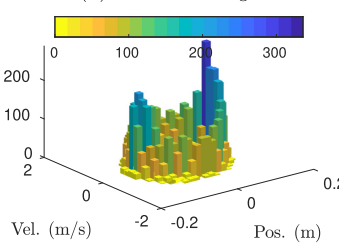

(G) - ID8 - 3D Histogram

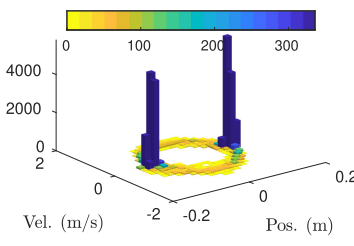

(D) - ID2 - KDE

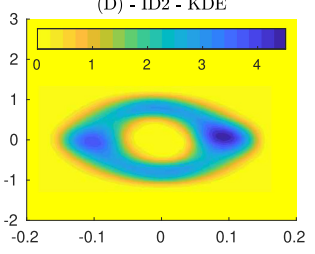

(H) - ID 8 - KDE

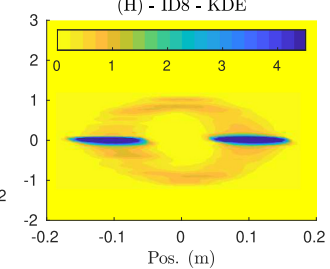

Fig. 15. Experimental phase planes, histograms, and densities for participant 10 and a distance of $212 \mathrm{~mm}$. (A) shows the recorded phase plane trajectory. (B) is a 2D histogram representation of the phase plane, generated with a grid of 30 by 30 bins. The scale represents the number of data points in each bin. (C) is a $3 \mathrm{D}$ histogram where the height of the bars corresponds to bin count, as in the 2D version. (D) is the KDE of the probability density of the corresponding data. The top row (A, B, C, D) shows data for ID 2, whereas ID 8 is displayed at the bottom $(\mathrm{E}, \mathrm{F}, \mathrm{G}, \mathrm{H})$. The horizontal and vertical axes correspond to pointer position and velocity, respectively. For $\mathrm{C}$ and $\mathrm{G}$, these two axes appear now in the horizontal plane and the height of the bins in the vertical one.

to visualise probability densities is simply to binning the data into discrete blocks to create $2 \mathrm{D}$ and 3D histograms to expose the variability observed in the data. The availability of such probability density estimates would allow us to predict the likelihood of a given trajectory that a specific participant might take for a particular task. In addition to simple histograms, we can estimate continuous density functions. In this case, the densities are estimated by Kernel Density estimates (KDE). KDE is a non-parametric approach to estimate the probability density function of a random variable, and is essentially a data smoothing problem where continuous inferences about the population are made, based on a finite data sample [Parzen 1962; Rosenblatt 1956]. To do this, the first step was to create densities based on the recorded time-series of all the simulations (200 simulations in total), in particular the pointer position and its velocity. With this information, a phase-plane density was created.

To establish a comparison, histograms and KDE densities generated from the experimental data from Participant 10 are presented first, followed by similar visualisations generated from the IC models that were identified. In Figure 15, a summary of different visualisations of the experimental data is shown, for ID 2 and 8, when a distance between targets is $212 \mathrm{~mm}$. The phase plane is shown for reference (Figure 15(A), (E)), followed by a 2D histogram representation that was generated based on the phase plane time-series (Figure 15(B), (F)). These heatmap-like figures in 2D can be extended to obtain a 3D representation as shown in Figure $15(C)$ and $(G)$, where the height of each histogram bar represents the bin count in a $30 \times 30$ grid. It is possible to observe how, as the phase plane trajectory approaches the targets, the count increases showing areas and bars in a dark blue colour. This is a clear indication of the participant slowing down to land on the target which results in more data points around these areas. Finally, in Figure 15(D) and (H), a 2D KDE is generated to have a probabilistic representation of the data.

The identified IC models were used to run 200 simulations using the multiple controller approach to build a density representation. First, we show a summary with the corresponding phase plane, 2D-3D histograms and densities for ID 2 and ID 8 for a distance of $212 \mathrm{~mm}$ in Figure 16, 

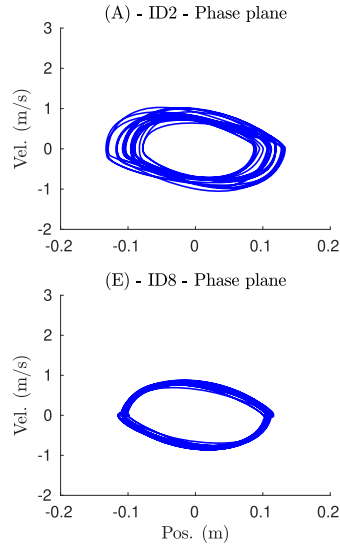

(B) - ID2 - 2D Histogram

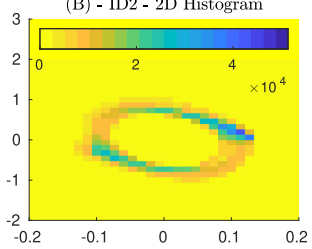

(F) - ID8 - 2D Histogram

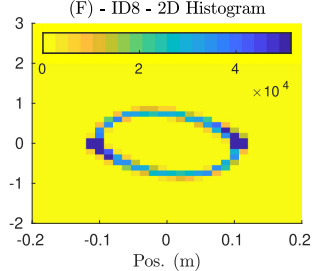

(C) - ID2 - 3D Histogram

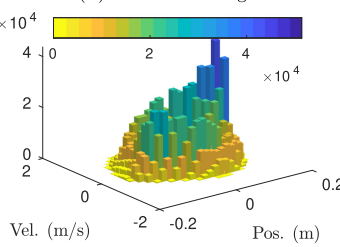

(G) - ID8 - 3D Histogram

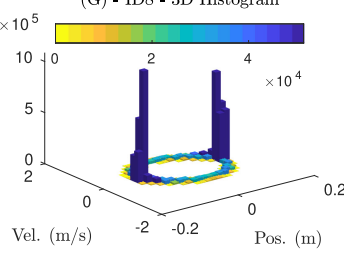

(D) - ID2 - KDE

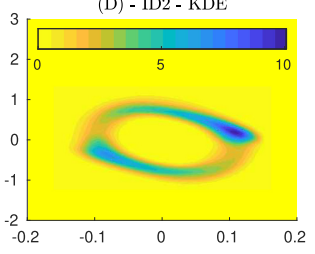

(H) - ID8 - KDE

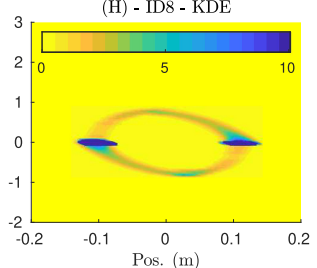

Fig. 16. Simulated phase planes, histograms, and densities for participant 10 and a distance of $212 \mathrm{~mm}$. A total of 200 simulations, using the multiple controller approach, were used to generate the same type of visualisations as in Figure 15. The simulated densities have similar shapes to the experimental ones in Figure 15, showing also higher higher density values around the targets.

which is comparable to Figure 15, followed by the simulated densities for all distances and conditions (Figure 17).

The simulated densities show a similar shape to the experimental counterparts, with the variability also present for low ID values such as 2 (A, B). Similarly, the difficult conditions ( $G$ and $\mathrm{H})$ also show high density values around the targets showing how the IC model trajectories also spend more time in the vicinity of the target before accelerating in the opposite direction.

\subsection{Kullback-Leibler Divergence for Repeated Simulations}

The information from the repeated simulations can be used to calculate a distance measure that evaluates how much of the observed experimental distributions is captured by the simulated realisations of the phase planes. To measure the similarity between these, we used a Kullback-Leibler (KL) divergence measure (also called relative entropy). The KL divergence is a measure of how one probability distribution differs from a second, reference probability distribution. For discrete probability distributions $P$ and $Q$ defined on the same probability space, $\mathcal{X}$, the KL divergence from $Q$ to $P$ is

$$
D_{K L}(P \| Q)=\int p(x) \log \left(\frac{p(x)}{q(x)}\right) d x,
$$

where $p$ and $q$ denote the probability densities of $P$ and $Q$. We took the approach described in Wang et al. [2009], using the associated software ${ }^{6}$ to quantify the information loss when compared against the experimental data. We also quantified the loss if the continuous2ol controller [Müller et al. 2017] is used to generate an individual set of simulated trajectories.

The KL divergence values were computed for 20 simulations of the IC, for a distance between targets of $212 \mathrm{~mm}$ and for all participants and conditions. To obtain each value, two vectors of samples coming from the simulated time-series of the pointer position and velocity, i.e., the phase plane information, were compared against the corresponding experimental time-series. The reported values for IC represent the mean of the $20 \mathrm{KL}$ values of each simulation run. The mean of the resulting $20 \mathrm{KL}$ values for each participant was computed. This is the reported value IC in Figure 18, which shows a comparison of the mean KL values over 20 simulation runs of IC and

${ }^{6} \mathrm{https}$ //github.com/slaypni/universal-divergence. 


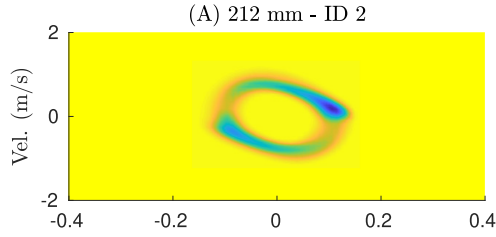

(C) $212 \mathrm{~mm}$ - ID 4

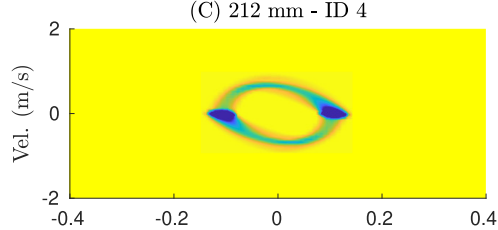

(E) $212 \mathrm{~mm}$ - ID 6

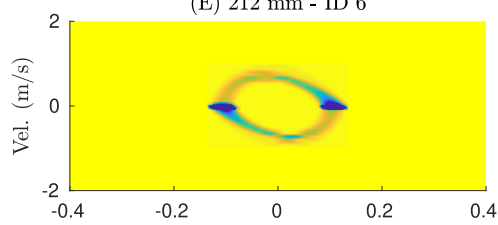

(G) $212 \mathrm{~mm}$ - ID 8

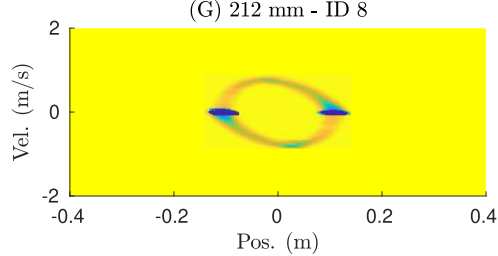

(B) $353 \mathrm{~mm}$ - ID 2

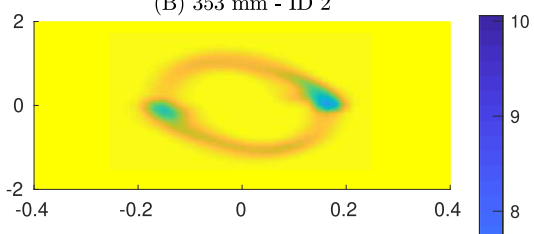

(D) $353 \mathrm{~mm}$ - ID 4

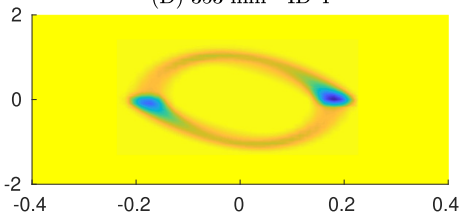

(F) $353 \mathrm{~mm}$ - ID 6

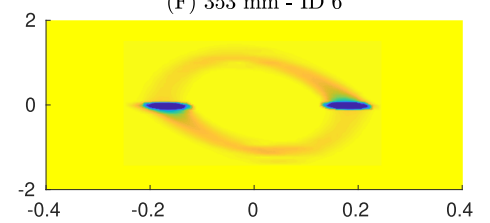

(H) $353 \mathrm{~mm}$ - ID 8

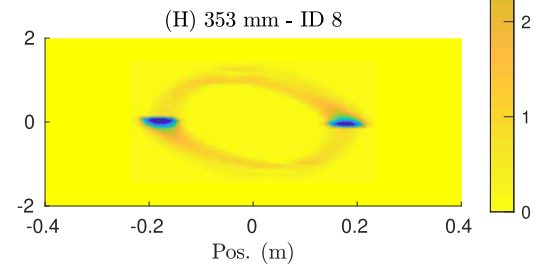

Fig. 17. Simulated KDE for participant 10 in all conditions and distances. (A), (C), (E), and (G) correspond to a distance of $212 \mathrm{~mm}$ whereas (B), (D), (F), and (H) are for $353 \mathrm{~mm}$. The different conditions are represented by each row of plots, with ID 2 shown at the top and finishing with ID 8 at the bottom. The horizontal and vertical axes in the figure represent pointer position and velocity, respectively. The simulated KDEs have lower values for ID 2 in both distances (A and B) since the participant uses a more cautious approach to hit the target, slowing down considerably, which leads to more data points in these regions.

a simulation of the $2 \mathrm{ol}$ continuous controller. Each subplot corresponds to a specific ID, starting with ID 2 on the left side (Figure 18(A)), ending with ID 8 on the right. The subplots show the KL values on the vertical axis and the two controllers are represented on the horizontal axis (IC on the left, 2ol on the right). The slope of each line (one per participant), shows how different the KL value is for a specific condition. A larger number means that the information loss is greater, therefore a small number indicates that the distribution from the simulation is closer to the observed distribution of the experimental data.

Most lines in Figure 18 have a positive slope except for two participants (P2 and P4) in ID 2 (Figure 18(A)). To gain more insight into the KL-divergence measure, view the phase plane responses for these participants in Figure 11, which does show repeated divergence in the model behaviour. The positive slopes show the KL divergence for IC are typically lower than for the 2ol controller, indicating a better fit to the distribution. Overall, the divergences for both controllers in ID 2 are smaller compared to the rest of the conditions (Figure 18(B), (C), (D)) in part because although ID 2 results are more variable than others, the variability itself is relatively consistent, making it easier to model. ${ }^{7}$ The variability of individual participants can be seen in Figure 19,

\footnotetext{
${ }^{7}$ Table 1 in the supplementary material contains all the values that were used to obtain Figure 18.
} 

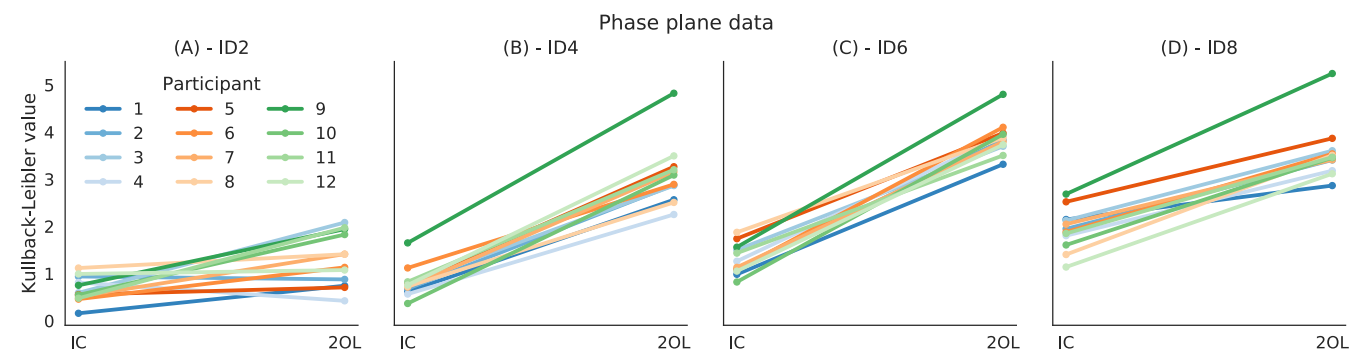

Fig. 18. KL divergence values of IC and 2ol controllers. (A), (B), (C), and (D) show the KL value, in the vertical axis, for all participants in conditions ID 2, ID 4, ID 6, and ID 8, respectively. Each line corresponds to a participant, where the left end of the line is the value for IC and the right end point represents the value of the 2ol controller. For IC, individual $\mathrm{KL}$ values were registered from 20 different simulation runs, from which a mean value was calculated. The mean is reported in this figure. The positive slopes of most of the lines indicate that IC provides a better fit to the observed distribution of the experimental data.

again compare the size and variability of divergence with the phase portrait plots for the same participants in Figure 11. The boxplots give a clear indication that overall the KL divergence tends to be lower for lower IDs, and that more difficult tasks have a higher KL divergence, and a larger variance in that divergence. Overall, the easier tasks result in lower KL values for IC than $2 \mathrm{ol}$ for all participants, apart from ID 2 for P2, P4, P5, P8, and P12.

\section{LOW-DIMENSIONAL VISUALISATIONS OF MODEL STRUCTURE}

While the models may appear complex at first sight, it is possible to explore a simplified model space in two ways: (1) experimentation with optimisation of a reduced subset of model parameters and (2) use of low-dimensional visualisation techniques to map the full parameter space to one or two dimensions to investigate the range of model behaviour for the different tasks. We combine both approaches in this section.

\subsection{Optimisation of a Reduced Parameter Set}

Analysis of the variation in model parameters suggested that we could potentially fix some of the values for all users, and restrict the identification process to a subset of parameters. This helps us understand the key factors in the resulting models. We therefore repeated the modelling process described earlier, but restricted the optimisation process to a reduced parameter set, where only $\mathrm{Q}_{c 1}, \mathrm{Q}_{c 2}, q$, and $\mathrm{A}_{p}$ were optimised, leaving out the minimum-open loop interval $\Delta_{o l}^{\mathrm{min}}$, the observer gain $Q_{o}$ and the rest of the diagonal entries in $Q_{c}$. The parameters that were left out from the optimisation took the following fixed values: $Q_{o}=10, Q_{c 3}=Q_{c 4}=1, \Delta_{o l}^{\min }=0.3 \mathrm{sec}$.

To measure how well the optimisation using a reduced parameter set would compare to the full set optimisation in terms of quality of fit, we decided to evaluate the cost function in Equation (37) for each individual slice. In addition to this, we evaluated the cost function when the controller obtained from each of the slices was simulated against all trials of the condition. Figure 20 shows the results of this comparison, where Figure 20(A) and (B) show the cost per slice for all participants for the full and reduced parameter sets, respectively; similarly, Figure 20(C) and (D) show the cost over all trials for the two sets.

From Figure 20(A) and (B), we can see that the reduced parameter optimisation does not represent too much of a tradeoff in quality of fit, since the cost per slice values are very similar with the most noticeable difference in ID 2, where the reduced set shows slightly higher cost values. The cost over the entire trials (Figure 20(C), (D)) for the two sets is very similar in both value and shape of the distributions; however, when compared to the cost per slice, we can see that it 

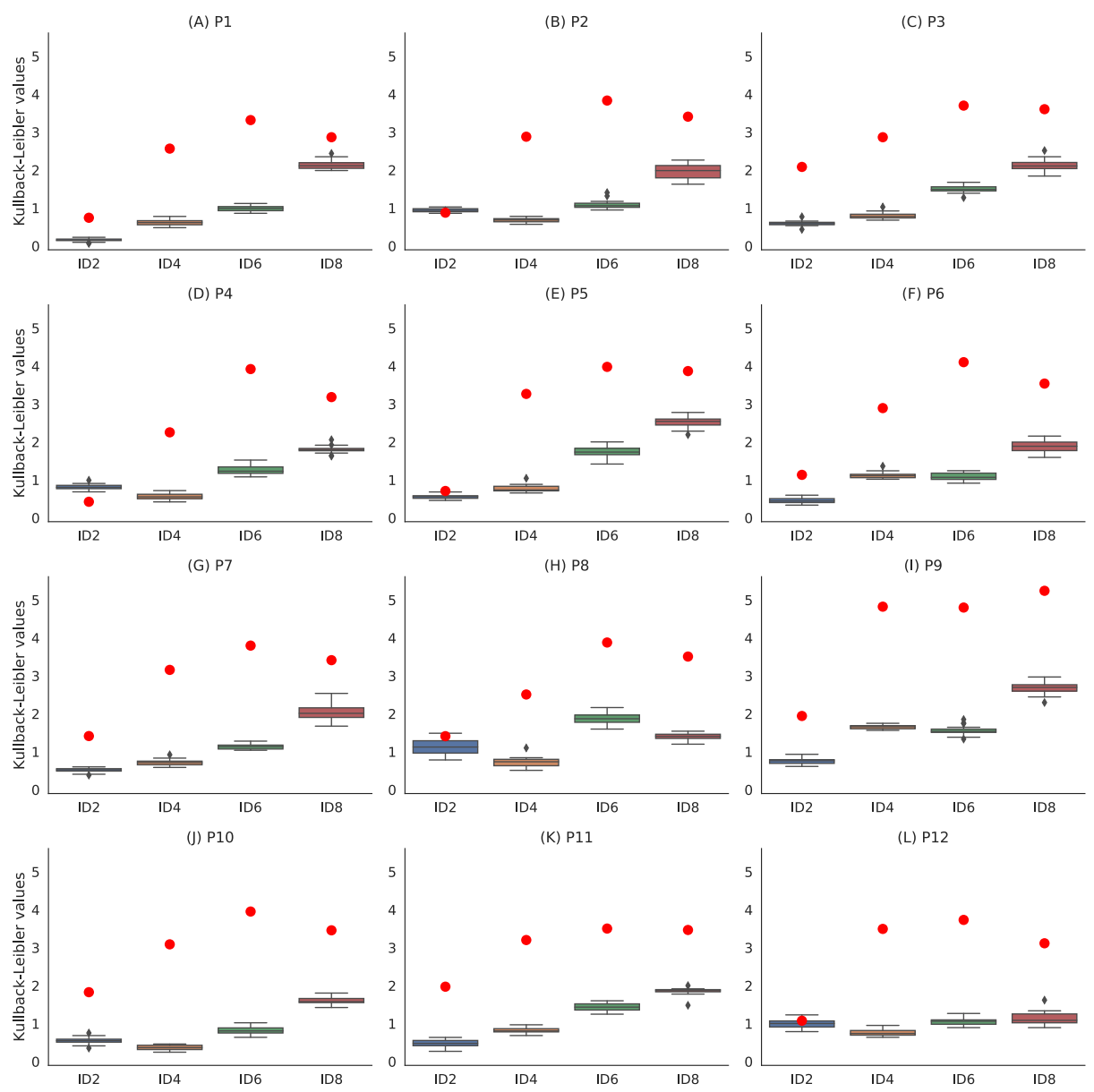

Fig. 19. Boxplots of the $K L$ divergence values for all participants and conditions. The results for each participant are grouped according to the ID of the task (horizontal axis). Overall, the easier tasks result in lower KL values for all participants. Red circles associated with each boxplot represent the value of the $2 \mathrm{OL}$ result for a particular condition. If the red dot is above the upper whiskers of the boxplot, this means that it is above the distribution of the IC results, indicating the significance of the difference between the conditions.

is generally higher across all conditions. This is an expected consequence of simulating a single controller against all trials in a condition and the main reason to use the switching control strategy introduced in Section 7.3.

The ability to successfully represent the data with only four parameters identified from the experimental data, may help reassure readers that the models can capture general properties of pointing behaviours, even in variable conditions such as ID 2, and is not "overfitting" the experimental data. The distributions of these four parameters are shown in Figure 21.

In Figure 21(A), we can see how $\mathbf{Q}_{c 1}$ has more compact distributions compared to the same figure for the full set of parameters (Figure 7(A)), whereas $Q_{c 2}$ in Figure 21(B) still shows elongated bimodal distributions that are comparable in shape to those in Figure 7(B). The threshold $q$ and the mismatch gain $\mathbf{A}_{p}$ are shown in Figure 21(C) and (D), respectively. The mismatch gain $\mathbf{A}_{p}$ in the reduced parameter set has now a longer distribution that spans from -0.1 to 0.1 for ID 2 when 
(A) - Full

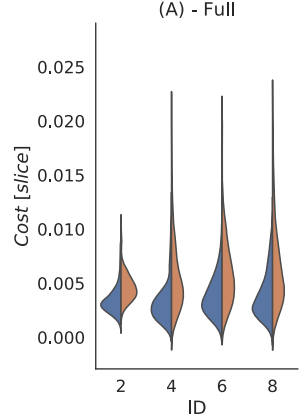

(B) - Reduced

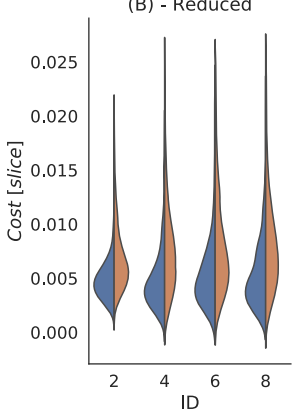

(C) - Full

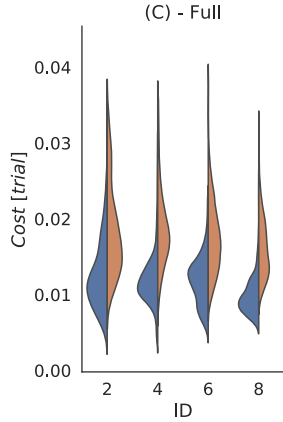

(D) - Reduced

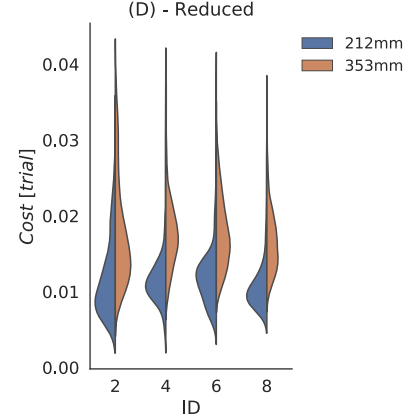

Fig. 20. Slice and trial cost for the reduced and full parameter sets in each condition. (A) The cost per slice for the full parameter set, (B) cost per slice for the reduced parameter set, (C) cost over all trials for the full parameter set, and (D) cost over all trials for the reduced parameter set, are shown as violin plots for all participants and categorised by ID on the horizontal axis. The results are also grouped according to the two values of distance between targets used in the experiment (left: $212 \mathrm{~mm}$ and right: $353 \mathrm{~mm}$ ).

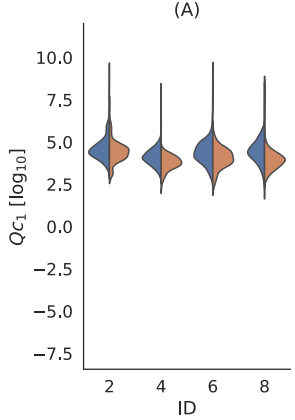

(B)

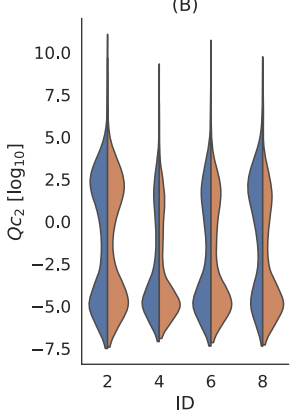

(C)

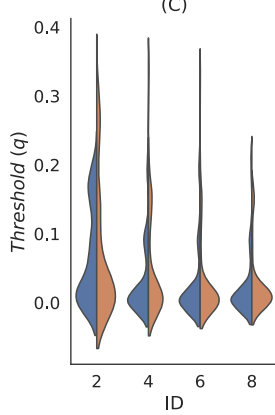

(D)

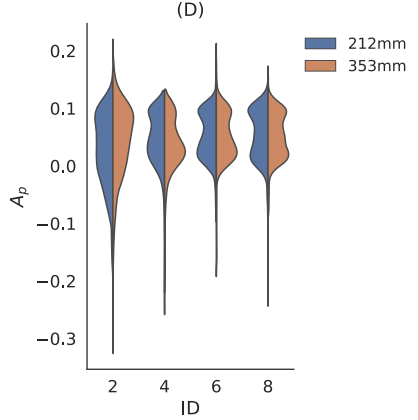

Fig. 21. Optimised controller parameters (for a reduced set) for each condition. (A) $\mathrm{Q}_{c 1},(\mathrm{~B}) \mathrm{Q}_{c 2}$, (C) the threshold $q$, and (D) the mismatch gain $\mathrm{A}_{p}$ are shown as violin plots including data of all participants and categorised by ID (horizontal axis). The results are also grouped according to the two values of distance between targets used in the experiment (left: $212 \mathrm{~mm}$ and right: $353 \mathrm{~mm}$ ).

compared to its distribution in the full parameter set (Figure 6(B)), which is more compact and centered around 0.1 .

The impact of the optimised $\mathbf{Q}_{c}$ values is on the gain parameters, therefore in Figure 22, we show all elements of the state-feedback gain vector $\mathbf{k}$ which is computed via LQR using the matrix $\mathrm{Q}_{c}$ as a design parameter.

The distributions of the state-feedback gain vector $\mathbf{k}$ for the reduced parameter set, shown from left to right in Figure 22, are more compact when compared to the corresponding gains of the full parameter set optimisation in Figure 8; on the other hand, the overall trend is similar for both parameter sets where $\mathbf{k}_{1}$ and $\mathbf{k}_{2}$ are both higher than $\mathbf{k}_{3}$ and $\mathbf{k}_{4}$, and $\mathbf{k}_{2}$ being lower in general than $\mathbf{k}_{1}$.

\subsection{Visualisation of Low-Dimensional Embeddings with UMAP}

Traditionally approaches such as Principal Components Analysis (PCA) have been used to visualise reduced dimensional representations of high-dimensional data. PCA is a linear approach, which limits its power. In this section, we use a modern non-linear algorithm, Uniform Manifold Approximation and Projections (UMAP) [McInnes et al. 2018], to arrange the reduced, fourdimensional model parameter vectors on a two-dimensional space. This allows us to visualise the 
(A)

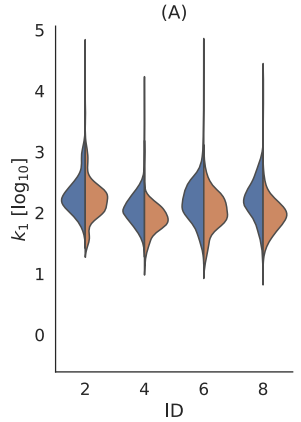

(B)

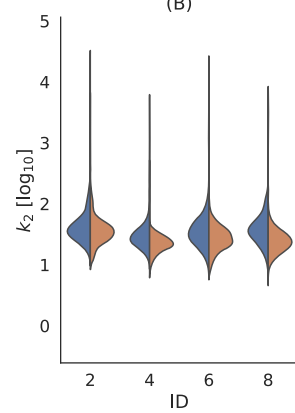

(C)

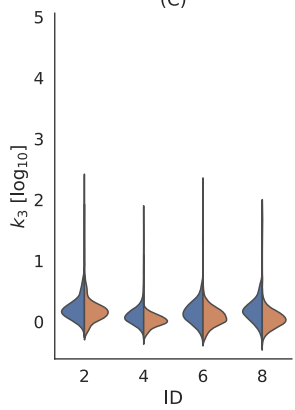

(D)

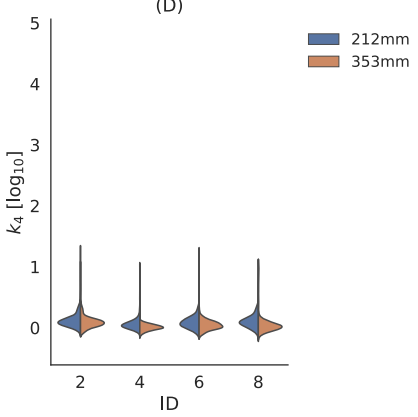

Fig. 22. Resulting controller gains $\mathbf{k}$ (for a reduced set) for each condition. This figure shows the four elements of the gain vector $\mathbf{k}$ for all participants and segmented by ID (horizontal axis). The results for $\mathbf{k}_{1}, \mathbf{k}_{2}, \mathbf{k}_{3}$, and $\mathbf{k}_{4}$ are shown from left to right in A, B, C, and D, respectively. Each violin plot introduces the results for the two distances between targets (left, in blue: $212 \mathrm{~mm}$ and right, in brown: $353 \mathrm{~mm}$ ) side by side.
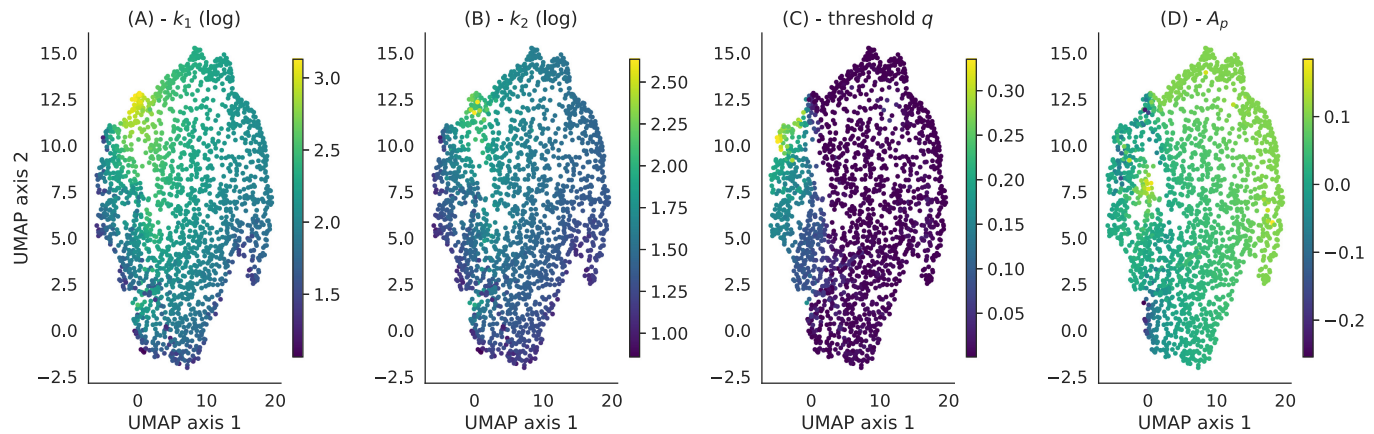

Fig. 23. Uniform Manifold Approximation using a 2D embedding, coloured using the value of each parameter. From left to right, we have the state-feedback gains $\mathbf{k}_{1}$ and $\mathbf{k}_{2}(\mathrm{~A}, \mathrm{~B})$, followed by the threshold $q$ and the mismatch gain $A_{p}(\mathrm{C}, \mathrm{D})$. Both axes represent the two dimensions of the UMAP embedding, which contains the data for all participants, IDs and distances between targets. This gives an indication of the typical ranges and combinations of parameters obtained from the reduced set optimisation. $\mathbf{k}_{1}$ and $\mathbf{k}_{2}$ are higher for the samples in the top left region of the embedding. Low levels of the threshold $q$ are quite common except for a small patch of samples located on the left side of the embedding. The mismatch gain $A_{p}$ has higher values towards the right side, with a small patch of negative values at the bottom.

smooth changes in model parameters over the space, as shown in Figure 23 and helps us associate them with qualitative changes in pointing behaviour, as shown in Figure 24. This gives an impression of the locations of specific users in parameter space for a given condition, and the impact of different parameters on time-series variability. In general, the individual users have behaviours which are spread widely around the space, with only extreme behaviours (e.g., P2) in more distinct distributions.

In Figure 23, the resulting two-dimensional embedding obtained using UMAP is displayed, while its samples are coloured according to the value of the two first state-feedback gains $\mathbf{k}_{1}$ and $\mathbf{k}_{2}$ (A and $\mathrm{B}$ ), as well as the threshold $q$ and mismatch gain $\mathrm{A}_{p}(\mathrm{C}$ and $\mathrm{D})$. This gives insight on how the relevant parameters change in the $2 \mathrm{D}$ space. For instance, the samples for $\mathbf{k}_{1}$ and $\mathbf{k}_{2}$ have higher values in general towards the top left corner of the embedding. The threshold $q$ is generally low except for a patch of samples on the top left corner, and $A_{p}$ shows the lowest values on the left half of the embedding, specially at the bottom. 
(A) - P2 - ID2 - 212mm

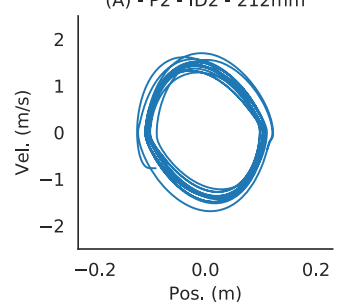

(B) - P5 - ID2 - $212 \mathrm{~mm}$

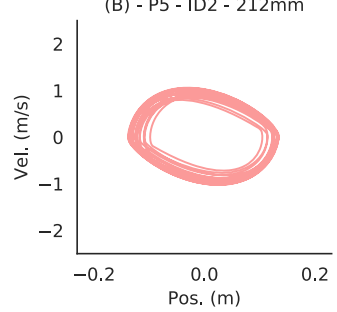

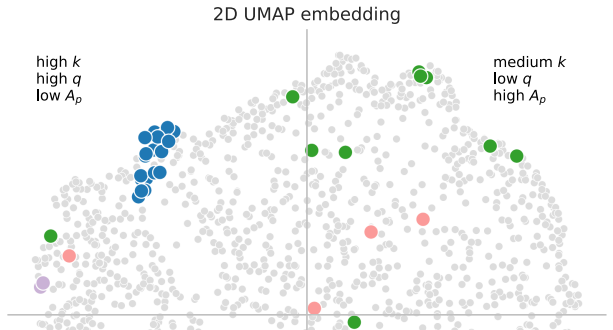

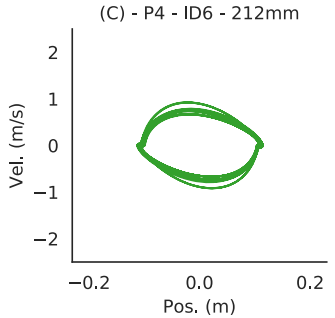

(D) - P9 - ID6 - 212mm
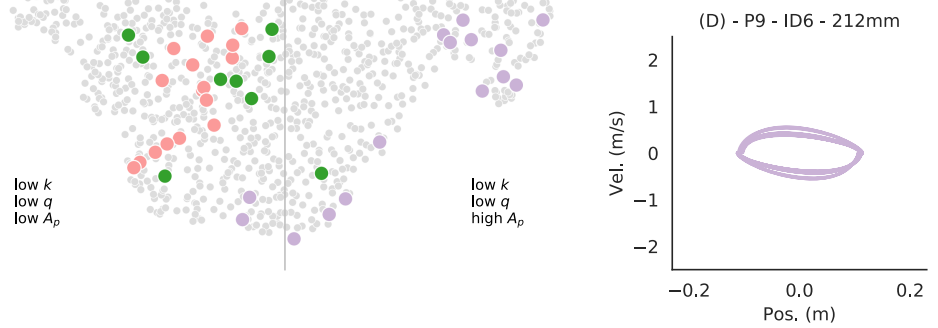

Fig. 24. Some samples of individual users and conditions for the $212 \mathrm{~mm}$ distance between targets and the associated phase planes. Participants 2 (A, blue) and 5 (B, pink) for an ID of 2 are shown in the left column, while participants 4 (C, green) and 9 (D, light purple) are displayed on the right. The corresponding samples in the 2D UMAP embedding are highlighted using the same colours. The embedding is classified roughly in four regions with approximate features of the optimised parameters, such as high and low values of $\mathbf{k}, q$ and $\mathrm{A}_{p}$. Note how the high variability ID 2 case is in the high gain region, while e.g., the P5 data are spread more evenly in the diagonal axis from low $k$, low $A_{p}$ to medium $k$, high $A_{p}$.

From the parameter mappings in Figure 23, we generated Figure 24 which displays the type of phase planes that IC would generate for a specific condition and participant, while looking at the position of the associated samples over the space. The two-dimensional UMAP embedding is shown in the centre, and relevant samples of different colours are overlapped. The corresponding phase planes are shown on the left and right columns relating the pointer position against velocity.

Participant 2 (A, blue) is clearly clustered in the upper left corner of the embedding for an ID of 2, which coincides roughly with the high gain $\mathbf{k}$, high threshold $q$, and low $\mathbf{A}_{p}$ region of the space. The phase plane for participant 2 shows trajectories of high velocity (vertical axis), giving rise to a more round phase plane. If this is compared with participant 5 in pink (B), for the same ID and distance, the phase plane does not show the same level of velocity as participant 2 and the associated samples lie mostly on the bottom left region of the embedding that corresponds to low levels for all of the parameters. This suggests that the state-feedback gains have an effect on the velocity profile of the participants.

In similar fashion, participant 4 (C, green) and 9 (D, light purple) are compared for an ID of 6 on the right column of Figure 24. A more precise trajectory is observed for both participants since the targets are smaller for this condition, but if we look at their samples on the embedding, we can see that participant 4 has samples on both the top right and bottom left sections of the embedding, whereas participant 9 is more localised around the bottom right section. These two participants have a considerable amount of samples in the high mismatch gain $\mathrm{A}_{p}$ regions of the embedding.

The easiest condition, i.e., ID 2, has greater degrees of freedom compared to the rest and separates out participants the most in terms of their position in the $2 \mathrm{D}$ embedding. This is shown in Figure 25, where the samples for all participants are displayed for ID 2 and a distance of $212 \mathrm{~mm}$. Participants 2 (blue), 4 (green), and 6 (red) tend to have clusters of samples in the top left region of the embedding, while participants 9 (light purple) and 12 (brown) tend to be in the lower half. 


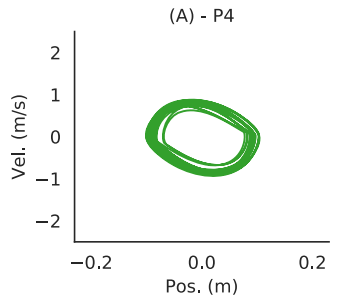

(B) - P6

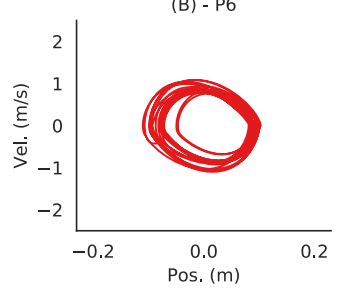

2D UMAP embedding - ID 2 - Distance: $212 \mathrm{~mm}$

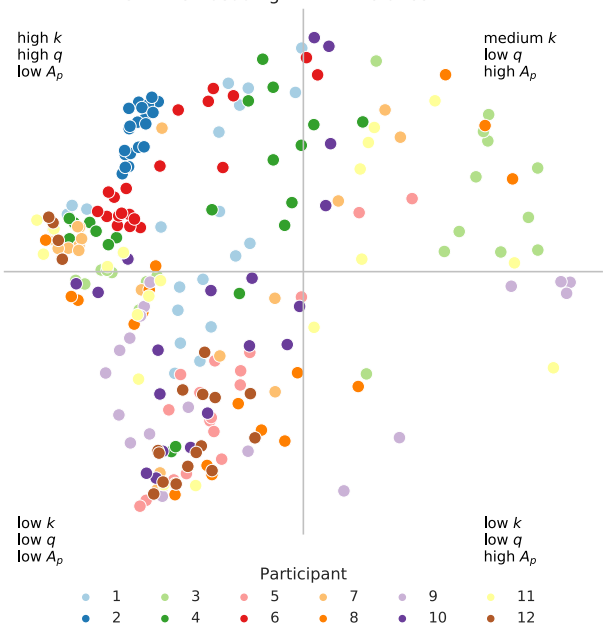

(C) - P9

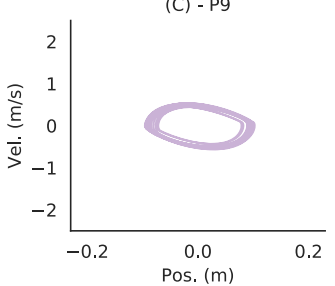

(D) - P12

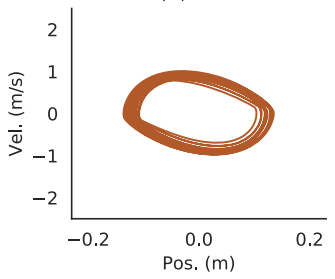

Fig. 25. Samples of a 2D UMAP embedding corresponding to all participants, an ID of 2, and a distance of 212 $\mathrm{mm}$. Each participant is shown in a different colour. The embedding is classified roughly in four regions with approximate features of the optimised parameters, such as high and low values of $\mathbf{k}, q$ and $\mathrm{A}_{p}$ as in Figure 24 Participants 2 (blue), 4 (green), and 6 (red) stand out with most samples in the areas of high state-feedback gains $\mathbf{k}$ and threshold $q$, located in the top left region of the embedding. Participants 9 (light purple) and 12 (brown) have most samples in the bottom left region where the values for all parameters are relatively low. The phase planes for participants $4,6,9$, and 12 are shown in A, B, C, and D, respectively.

The corresponding phase planes for participants 4, 6, 9, and 12 are shown in Figure 25(A)-(D), respectively. The samples for all participants are more mixed for the rest of the conditions. ${ }^{8}$

\section{DISCUSSION}

\subsection{Key Conceptual Advantages of This Model}

Our results show that IC provides a viable model of mouse movements. In particular, we show that existing CC models of mouse movements (e.g., Müller et al. [2017]), while a significant advance on previous models with no dynamics, provide a highly simplified view of movement dynamics in HCI tasks. In particular, these previous models assume that humans can react to computer feedback continuously. On a high level, humans can appear to react to feedback continuously, such that CC models can be viewed as a (rough) approximation of human behaviour. However, newer research suggests that humans may not be able to react truly continuously because of the psychological refractory period [Loram et al. 2014]. Instead, IC can masquerade as CC, such that in some cases, both behaviours cannot be distinguished. However, IC is a more general, and more physiologically plausible, explanation of behaviour. In particular, it can explain phenomena that can not be explained by CC, including the following:

Variability: The parametric, CC models proposed in Müller et al. [2017] are unable to replicate human variability. Instead of replicating the variance of human movements, these models always recreate the same movement given the same initial conditions and task. CC models could be augmented by injecting sensorimotor noise into the control loop to replicate human variability. However, intermittency of control is a physiologically plausible additional noise source

\footnotetext{
${ }^{8}$ Figure 1 of the supplementary material shows the 2D UMAP embedding for all participants and conditions, and a distance of $212 \mathrm{~mm}$.
} 
[Gollee et al. 2017] that, coupled with the model's internal dynamics, can explain the variability in pointing movements well.

Submovements: One practical consequence of the simplification associated with CC models is their fundamental inability to explain or replicate submovements. While humans demonstrate identifiable submovements in difficult aimed movements, e.g., in mouse pointing, CC models predict a single smooth movement towards the target. Submovements are a characteristic feature of aimed movements, and the inability to replicate these represent a fundamental shortcoming of CC models. However, this shortcoming is solved through the IC models proposed in this article. IC provides a plausible explanation of the physiological mechanisms underlying the existence of submovements, and our work shows that it is also able to replicate movements with submovements empirically. The threshold functions used in this article are simple fixed levels, but we anticipate that future work can capture more structure by having more complex classifiers which are a function of both the error and the state.

Predictive models in users: One particularly interesting aspect of IC models is that they posit that users maintain an internal model of the computer interface dynamics and predict the feedback they will receive from the computer interface in the future. They only change their control when the received feedback deviates from their internal prediction. This maintenance of an internal model is in line with developments in neuroscience, e.g., Wolpert and Ghahramani [2000]. More awareness among HCI designers of the fact that control is intermittent and that humans control according to an internal model of the interface dynamics has the potential to fundamentally change the design of the feedback of computer interfaces. Computer interfaces evolve according to an internal state, and aspects of this state are communicated as feedback to the user. The ability of the user to construct an internal model of the state dynamics, as well as to observe the state from feedback is crucial for their ability to close the IC loop more rarely, potentially freeing cognitive resources. Designers of feedback of computer interfaces should pay more attention to making as much as possible of the full state of the computer interface observable, e.g., by visualising not only the positions but also the velocities of virtual objects. The congruence of feedback received from the interface and the feedback predicted by the user is important not only for the control performance, but also for psychological phenomena such as the agency perceived by the user over the interface [Seinfeld et al. 2020].

Furthermore, IC models previously assumed that the system matched hold was a perfect approximation of the controlled system. In this article, we introduce the concept of the mismatch gain, $A_{p}$, to account for the difference between the internal model (which is used in the hold and in the predictor) and the real system (which is subject to disturbances). This results in the ability of our model to represent the undershooting and overshooting trajectories which are characteristic of this task. We also extend our identification approach to be suitable for a reference tracking task rather than compensatory control, as used previously [Gollee et al. 2017].

\subsection{Limitations of the Model}

This article presents initial work building on experimental data for one-dimensional pointing tasks of 12 test users in an artificial lab setting, with constant mouse gain, no visual distractors, and a limited range of ID and distance. In particular, our current model formulation assumes linear user interface dynamics, e.g., pointing with constant mouse gain. However, the model can be extended to the case of non-linear user interface dynamics, e.g., non-linear PTFs, via effectively local linearisation of a non-linear observer model. The case of a static non-linearity can be addressed by using its inverse, so that the overall plant appears to be linear. Further directions of future work involve generalising the model to everyday mouse interactions with typical levels of visual distractors on screen, and multivariable movement tasks (e.g., 2D targets of different shapes). However, we 
anticipate that the nature of the IC approach is naturally well suited to multivariate control models, allowing a flexible expansion to higher-dimensional interaction tasks. Similarly, the dynamic systems roots make it well suited for dynamic tasks such as Steering law tasks [Accot and Zhai 1997], allowing us to better model user behaviour in tunnel or trajectory following tasks, tracking of moving targets [Poulton 1974] and crossing-based interfaces [Accot and Zhai 2002]. It will also be of use in general gesture recognition models which can often be of significantly higher dimension.

\section{CONCLUSION}

We proposed IC models as a model for movement in HCI, investigating IC of pointing movements with a computer mouse.

We identified parameters of our model from data of a reciprocal pointing experiment. Simulation of our IC model shows that it can replicate human pointing movements well, given a small number of parameters. A significant difference from our previous work on CC models in Müller et al. [2017] is that IC models can both reproduce the empirical variability of human pointing movements and provide a physiologically plausible explanation for the variability, in the system dynamics coupled with the variability of triggering the sampling of feedback information. IC models are also inherently able to explain and predict submovements. We conclude that for the one-dimensional pointing task, IC models are physiologically plausible and have stronger empirical support than current CC models of movement in HCI.

The availability of identifiable, dynamic human control models, which also accurately capture the rich variability of human behaviour, will have an important role in the future design, testing, and analysis of HCI. While point-and-click interfaces have dominated interaction with computers for decades, they exploit only a tiny fraction of the richness of motion that humans are able to produce. Interfaces that only interpret the movement endpoint at the time of click throw away all information that was generated in the process of movement. This can include using the movement dynamics to give the system predictive information about what user is trying to achieve, what their emotional state is, or user identification, for security or personalisation purposes. (The dynamics of movement are much more difficult to counterfeit than, say, the static image of a signature.) In the future, interfaces that accumulate information over the whole interaction might go far beyond that. Future interfaces are also tightly integrated in the physical world and equipped with multitudes of sensors such as gyroscopes, accelerometers, and cameras. This would enable the interaction to generate richer reactions to the environment and more complex, engaging, dynamics, such as during physical scrolling or layout adjustments, especially in the case of Virtual Reality based on physics-based environment simulations.

The control perspective on interaction provides a unifying theoretical and modelling framework for the description, analysis and model-based design of interfaces. They enable the simulation of user behaviour in interaction, reducing the number of user studies necessary in the design phase. Such simulations allow us to gain insight into processes that are difficult to observe because they are internal to the user, and to design and optimise for them.

\section{AUTHORS' STATEMENT}

The results shown in this article are based on the Pointing Dynamics Dataset that was collected from a mouse pointing experiment described in Müller et al. [2017]. This new article introduces IC as a plausible framework for the modelling of user movement in the interaction with computers, and as a novel analytic and practical tool for HCI research and practice.

In IC, open-loop control trajectories are generated based on an internal estimate of the state of interactions using a predictive model of how this state will evolve over time. These openloop trajectories are updated only intermittently with feedback information from continuous 
observations of the systems. This IC perspective is more physiologically plausible as an explanation of human motor control than our previous $\mathrm{CC}$ perspectives of interaction with computers which were already published in ToCHI.

We examine the ability of IC to generatively reproduce and explain distinctive dynamical features of the pointing task such as the velocity profile and the implications for the variability observed across participants. The article demonstrates a better fit to the variability of mouse movement data than any previously published work. Because of the use of control methods designed for multidimensional control, this fundamentally new approach to human movement analysis is anticipated to generalise well to multidimensional movement tasks like gesture-based interfaces, and dynamic tracking tasks (e.g., targeting in gaming).

This is an original article that has not been submitted to any other journal or conference.

\section{REFERENCES}

Johnny Accot and Shumin Zhai. 1997. Beyond Fitts' law: Models for trajectory-based HCI tasks. In Proceedings of the ACM SIGCHI Conference on Human Factors in Computing Systems. ACM, New York, NY, 250.

Johnny Accot and Shumin Zhai. 2002. More than dotting the i's-foundations for crossing-based interfaces. In Proceedings of the SIGCHI Conference on Human Factors in Computing Systems. ACM, New York, NY, 73-80.

S. Aranovskiy, R. Ushirobira, D. Efimov, and G. Casiez. 2016. Modeling pointing tasks in mouse-based human-computer interactions. In Proceedings of the 2016 IEEE 55th Conference on Decision and Control. IEEE, Las Vegas, NV, 6595-6600.

Stanislav Aranovskiy, Rosane Ushirobira, Denis Efimov, and Géry Casiez. 2020. A switched dynamic model for pointing tasks with a computer mouse. Asian fournal of Control 22, 4 (2020), 1387-1400.

Myroslav Bachynskyi. 2016. Biomechanical Models for Human-Computer Interaction. Ph.D. Dissertation. Universität des Saarlandes Saarbrücken.

Myroslav Bachynskyi, Gregorio Palmas, Antti Oulasvirta, and Tino Weinkauf. 2015. Informing the design of novel input methods with muscle coactivation clustering. ACM Transactions on Computer-Human Interaction 21, 6 (2015), 1-25.

Ravin Balakrishnan. 2004. 'Beating' Fitts' law: Virtual enhancements for pointing facilitation. International fournal of Human-Computer Studies 61, 6 (2004), 857-874.

R. C. Barrett, E. J. Selker, J. D. Rutledge, and R. S. Olyha. 1995. Negative inertia: A dynamic pointing function. In Proceedings of the Conference Companion on Human Factors in Computing Systems. ACM, New York, NY, 316-317.

Paul M. Bays and Daniel M. Wolpert. 2007. Computational principles of sensorimotor control that minimize uncertainty and variability. The fournal of Physiology 578, 2 (2007), 387-396.

Nikhil Bhushan and Reza Shadmehr. 1999. Computational nature of human adaptive control during learning of reaching movements in force fields. Biological Cybernetics 81, 1 (1999), 39-60.

Magali Billon, Reinoud J. Bootsma, and Denis Mottet. 2000. The dynamics of human isometric pointing movements under varying accuracy requirements. Neuroscience Letters 286, 1 (2000), 49-52.

Renaud Blanch, Yves Guiard, and Michel Beaudouin-Lafon. 2004. Semantic pointing: Improving target acquisition with control-display ratio adaptation. In Proceedings of the SIGCHI Conference on Human Factors in Computing Systems. ACM, New York, NY, 519-526.

R. J. Bootsma, L. Fernandez, and D. Mottet. 2004. Behind Fitts' law: Kinematic patterns in goal-directed movements. International Journal of Human-Computer Studies 61, 6 (2004), 811-821.

Robin T. Bye and Peter D. Neilson. 2008. The BUMP model of response planning: Variable horizon predictive control accounts for the speed-accuracy tradeoffs and velocity profiles of aimed movement. Human Movement Science 27, 5 (2008), 771-798.

S. K. Card, T. P. Moran, and A. Newell. 1986. The model human processor: An engineering model for human performance. In Handbook of Perception and Human Performance, Vol. 2. Cognitive Processes and Performance. K. R. Boff, L. Kaufman, \& J. P. Thomas (Eds.), Wiley, pp. 1-35.

Géry Casiez and Nicolas Roussel. 2011. No more bricolage! Methods and tools to characterize, replicate and compare pointing transfer functions. In Proceedings of the 24th Annual ACM Symposium on User Interface Software and Technology. ACM, New York, NY, 603-614.

Géry Casiez, Daniel Vogel, Ravin Balakrishnan, and Andy Cockburn. 2008. The impact of control-display gain on user performance in pointing tasks. Human-Computer Interaction 23, 3 (2008), 215-250.

Olivier Chapuis, Renaud Blanch, and Michel Beaudouin-Lafon. 2007. Fitts' Law in the Wild: A Field Study of Aimed Movements. LRI Technical Repport Number 1480. Univ. Paris-Sud, Orsay.

Sung-Jung Cho, Roderick Murray-Smith, and Yeun-Bae Kim. 2007. Multi-context photo browsing on mobile devices based on tilt dynamics. In Proceedings of the 9th International Conference on Human Computer Interaction with Mobile Devices and Services. ACM, New York, NY, 190-197. 
Kenneth J. W. Craik. 1947. Theory of the human operator in control systems. I. the operator as an engineering system. British fournal of Psychology 38, 2 (1947), 56-61.

Kenneth J. W. Craik. 1948. Theory of the human operator in control systems; man as an element in a control system. The British fournal of Psychology. General Section 38, 3 (1948), 142-148.

E. R. F. W. Crossman and P. J. Goodeve. 1983. Feedback control of hand-movement and Fitts' law. The Quarterly fournal of Experimental Psychology 35, 2 (1983), 251-278.

Antonia F. de C. Hamilton, Kelvin E. Jones, and Daniel M. Wolpert. 2004. The scaling of motor noise with muscle strength and motor unit number in humans. Experimental Brain Research 157, 4 (Aug. 2004), 417-430.

Ashesh K. Dhawale, Maurice A. Smith, and Bence P. Ölveczky. 2017. The role of variability in motor learning. Annual Review of Neuroscience 40, 1 (2017), 479-498.

Parisa Eslambolchilar and Roderick Murray-Smith. 2004. Tilt-based automatic zooming and scaling in mobile devices - A state-space implementation. In Mobile Human-Computer Interaction - MobileHCI 2004. S. Brewster and M. Dunlop (Eds.). Springer Berlin Heidelberg, Berlin, 120-131.

Parisa Eslambolchilar and Roderick Murray-Smith. 2006. Model-based, multimodal interaction in document browsing. In Proceedings of the 3rd International Conference on Machine Learning for Multimodal Interaction. Springer-Verlag, Berlin, $1-12$.

Parisa Eslambolchilar and Roderick Murray-Smith. 2008. Control centric approach in designing scrolling and zooming user interfaces. International Journal of Human-Computer Studies 66, 12 (2008), 838-856.

A. Aldo Faisal and Simon B. Laughlin. 2007. Stochastic simulations on the reliability of action potential propagation in thin axons. PLOS Computational Biology 3, 5 (May 2007), 1-13.

A. Aldo Faisal, Luc P. J. Selen, and Daniel M. Wolpert. 2008. Noise in the nervous system. Nature Reviews Neuroscience 9,4 (April 2008), 292-303.

Jean-Daniel Fekete, Niklas Elmqvist, and Yves Guiard. 2009. Motion-pointing: Target selection using elliptical motions. In Proceedings of the SIGCHI Conference on Human Factors in Computing Systems. ACM, New York, NY, 289-298.

Florian Fischer, Miroslav Bachinski, Markus Klar, Arthur Fleig, and Jörg Müller. 2020. Reinforcement learning control of a biomechanical model of the upper extremity. arXiv:2011.07105. Retrieved from https://arxiv.org/abs/2011.07105.

Paul M. Fitts. 1954. The information capacity of the human motor system in controlling the amplitude of movement. fournal of Experimental Psychology 47, 6 (1954), 381-391.

U. Forssell and L. Ljung. 1999. Closed-loop identification revisited. Automatica 35, 7 (1999), 1215-1241.

P. Gawthrop, H. Gollee, and I. Loram. 2015. Intermittent control in man and machine. In Event-Based Control and Signal Processing, M. Miskowicz (Ed.). CRC, London, Chapter 14, 1-99.

P. Gawthrop, I. Loram, H. Gollee, and M. Lakie. 2014. Intermittent control models of human standing: Similarities and differences. Biological Cybernetics 108, 2 (April 2014), 159-68.

Peter Gawthrop, Ian Loram, Martin Lakie, and Henrik Gollee. 2011. Intermittent control: A computational theory of human control. Biological Cybernetics 104, 1-2 (2011), 31-51.

P. Gawthrop and L. Wang. 2007. Intermittent model predictive control. Proceedings of the Institution of Mechanical Engineers, Part I: fournal of Systems and Control Engineering 221, 7 (2007), 1007-1018.

P. Gawthrop and L. Wang. 2011. The system-matched hold and the intermittent control separation principle. International fournal of Control 84, 12 (2011), 1965-1974.

Peter J. Gawthrop and Liuping Wang. 2008. Towards model-based continuous-time identification of the human balance controller. IFAC Proceedings Volumes 41, 2 (2008), 11612-11617.

Peter J. Gawthrop and Liuping Wang. 2009. Event-driven intermittent control. International fournal of Control 82, 12 (2009), 2235-2248.

Henrik Gollee, Peter J. Gawthrop, Martin Lakie, and Ian D. Loram. 2017. Visuo-manual tracking: Does intermittent control with aperiodic sampling explain linear power and non-linear remnant without sensorimotor noise? The fournal of Physiology 595, 21 (2017), 6751-6770.

G. C. Goodwin, S. F. Graebe, and M. E. Salgado. 2001. Control System Design. Prentice Hall, New Jersey, NJ.

Julien Gori. 2018. Modeling the Speed-Accuracy Tradeoff Using the Tools of Information Theory. Ph.D. Dissertation. Paris Saclay.

Julien Gori, Olivier Rioul, and Yves Guiard. 2018. Speed-accuracy tradeoff: A Formal Information-Theoretic Transmission Scheme (FITTS). ACM Transactions on Computer-Human Interaction 25, 5 (2018), 1-33.

Yves Guiard. 1993. On Fitts's and Hooke's laws: Simple harmonic movement in upper-limb cyclical aiming. Acta Psychologica 82, 1 (1993), 139-159.

Yves Guiard and Michel Beaudouin-Lafon. 2004a. Fitts' law 50 years later: Applications and contributions from humancomputer interaction. International fournal of Human-Computer Studies 61, 6 (2004), 747-750.

Y. Guiard and M. Beaudouin-Lafon. 2004b. Target acquisition in multiscale electronic worlds. International fournal of Human-Computer Studies 61, 6 (2004), 875-905. 
Erik Hollnagel. 1999. Modelling the controller of a process. Transactions of the Institute of Measurement and Control 21, 4-5 (1999), 163-170.

E. Hollnagel and D. D Woods. 2005. Foint Cognitive Systems: Foundations of Cognitive Systems Engineering. CRC, Boca Raton, FL.

R. J. Jagacinski. 1977. A qualitative look at feedback control theory as a style of describing behavior. Human Factors: The fournal of the Human Factors and Ergonomics Society 19, 4 (1977), 331-347.

Richard J. Jagacinski and John M. Flach. 2003. Control Theory for Humans: Quantitative Approaches to Modeling Performance. Lawrence Erlbaum, Mahwah, New Jersey, NJ

Kelvin E. Jones, Antonia F. de C. Hamilton, and Daniel M. Wolpert. 2002. Sources of signal-dependent noise during isometric force production. Journal of Neurophysiology 88, 3 (Sept. 2002), 1533-1544.

David L. Kleinman. 1969. Optimal control of linear systems with time-delay and observation noise. IEEE Transactions on Automatic Control 14, 5 (1969), 524-527.

D. L. Kleinman, S. Baron, and W. H. Levison. 1970. An optimal control model of human response part I: Theory and validation. Automatica 6, 3 (1970), 357-369.

Sven Kratz, Ivo Brodien, and Michael Rohs. 2010. Semi-automatic zooming for mobile map navigation. In Proceedings of the 12th International Conference on Human Computer Interaction with Mobile Devices and Services. ACM, New York, NY, 63-72.

W. H. Levison, S. Baron, and D. L. Kleinman. 1969. A model for human controller remnant. IEEE Transactions on ManMachine Systems 10, 4 (1969), 101-108.

Ian Loram, Peter Gawthrop, and Henrik Gollee. 2015. Intermittent control of unstable multivariate systems. In Proceedings of the 2015 37th Annual International Conference of the IEEE Engineering in Medicine and Biology Society. IEEE, 1436-1439.

Ian D. Loram, Peter J. Gawthrop, and Martin Lakie. 2006. The frequency of human, manual adjustments in balancing an inverted pendulum is constrained by intrinsic physiological factors. The fournal of Physiology 577, 1 (2006), 417-432.

Ian D. Loram, Henrik Gollee, Martin Lakie, and Peter J. Gawthrop. 2011. Human control of an inverted pendulum: iIs continuous control necessary? Is intermittent control effective? Is intermittent control physiological? The fournal of Physiology 589, 2 (2011), 307-324.

I. D. Loram and M. Lakie. 2002. Human balancing of an inverted pendulum: Position control by small, ballistic-like, throw and catch movements. The fournal of Physiology 540, 3 (March 2002), 1111-1124.

I. D. Loram, Cornelis van de Kamp, H. Gollee, and P. J. Gawthrop. 2012. Identification of intermittent control in man and machine. Journal of the Royal Society Interface 9, 74 (Sept. 2012), 2070-84.

Ian David Loram, Cornelis van De Kamp, Martin Lakie, Henrik Gollee, and Peter J. Gawthrop. 2014. Does the motor system need intermittent control? Exercise and Sport Sciences Reviews 42, 3 (2014), 117-125.

I. Scott MacKenzie. 1992. Fitts' law as a research and design tool in human-computer interaction. Human-Computer Interaction 7, 1 (1992), 91-139.

Leland McInnes, John Healy, and James Melville. 2018. UMAP: Uniform Manifold Approximation and Projection for dimension reduction. arXiv:1802.03426. Retrieved from https://arxiv.org/abs/1802.03426.

D. E. Meyer, R. Abrams, S. Kornblum, C. E. Wright, and J. E. Smith. 1988. Optimality in human motor performance: Ideal control of rapid aimed movements. Psychological Review 95, 3 (1988), 340-370.

D. E. Meyer, J. E. Keith-Smith, S. Kornblum, R. A. Abrams, and C. E. Wright. 1990. Speed-accuracy trade-offs in aimed movements: Toward a theory of rapid voluntary action. In Attention and Performance XIII: Motor Representation and Control. M. Jeannerod (Ed.), Lawrence Erlbaum Associates, Inc, New Jersey, NJ, 173-226.

R. Christopher Miall. 1986. Simple or complex systems? Behavioral and Brain Sciences 9, 4 (1986), 734-734.

R. C. Miall, D. Jo Weir, Daniel M. Wolpert, and J. F. Stein. 1993. Is the cerebellum a smith predictor? fournal of Motor Behavior 25, 3 (1993), 203-216.

H. S. Milner-Brown, R. B. Stein, and R. Yemm. 1973. The contractile properties of human motor units during voluntary isometric contractions. The fournal of Physiology 228, 2 (1973), 285-306.

Jörg Müller, Antti Oulasvirta, and Roderick Murray-Smith. 2017. Control theoretic models of pointing. ACM Transactions on Computer-Human Interaction 24, 4, Article 27 (Aug. 2017), 36 pages.

Roderick Murray-Smith. 2018. Control theory, dynamics and continuous interaction. In Computational Interaction. A. Oulasvirta, P. O. Kristensson, X. Bi, and A. Howes (Eds.), Oxford University Press, Oxford, 17-42.

T. Navas and L. Stark. 1968. Sampling or intermittency in hand control system dynamics. Biophysics fournal 8, 2 (1968), 252-302.

P. D. Nielson. 1999. Influence of lntermittency and Synergy on Grasping. Motor Control 3, 3 (1999), 280-284.

Y. Oytam, P. D. Neilson, and N. O’Dwyer. 2005. Degrees of freedom and motor planning in purposive movement. Human Movement Science 24, 5-6 (2005), 710-730.

Eunji Park and Byungjoo Lee. 2020. An intermittent click planning model. In Proceedings of the 2020 CHI Conference on Human Factors in Computing Systems. ACM, New York, NY, 1-13. 
Emanuel Parzen. 1962. On estimation of a probability density function and model. Annals of Mathematical Statistics 33, 3 (1962), 1065-1076.

E. C. Poulton. 1974. Tracking Skill and Manual Control. Academic Press, New York, NY.

Philip Quinn, Sylvain Malacria, and Andy Cockburn. 2013. Touch scrolling transfer functions. In Proceedings of the 26th Annual ACM Symposium on User Interface Software and Technology. ACM, New York, NY, 61-70.

Philip Quinn and Shumin Zhai. 2018. Modeling gesture-typing movements. Human-Computer Interaction 33, 3 (2018), 234-280.

E. Ronco, T. Arsan, and P. J. Gawthrop. 1999. Open-loop intermittent feedback control: Practical continuous-time GPC. IEE Proceedings-Control Theory and Applications 146, 5 (1999), 426-434.

Murray Rosenblatt. 1956. Remarks on some nonparametric estimates of a density function. Annals of Mathematical Statistics 27, 3 (1956), 832-837.

Richard A. Schmidt, Howard N. Zelaznik, and James S. Frank. 1978. Sources of inaccuracy in rapid movement. In Information Processing in Motor Control and Learning. George E. Stelmach (Ed.), Academic Press, New York, NY, 183-203.

Sofia Seinfeld, Tiare Feuchtner, Antonella Maselli, and Jörg Müller. 2020. User representations in human-computer interaction. Human-Computer Interaction 0, 0 (2020), 1-39.

Reza Shadmehr and Sandro Mussa-Ivaldi. 2012. Biological Learning and Control: How the Brain Builds Representations, Predicts Events, and Makes Decisions. MIT Press, Cambridge, MA.

R. William Soukoreff and I. Scott MacKenzie. 2004. Towards a standard for pointing device evaluation, perspectives on 27 years of Fitts' law research in HCI. International fournal of Human-Computer Studies 61, 6 (2004), 751-789.

C. W. Telford. 1931. The refractory phase of voluntary and associative responses. fournal of Experimental Psychology 14, 1 (1931), 1-36.

Virginia Torczon. 1997. On the convergence of pattern search algorithms. SIAM fournal on Optimization 7, 1 (1997), 1-25.

Dari Trendafilov and Roderick Murray-Smith. 2013. Information-theoretic characterization of uncertainty in manual control. In Proceedings of the 2013 IEEE International Conference on Systems, Man, and Cybernetics. IEEE Computer Society, 4913-4918.

Cornelis van de Kamp, P. J. Gawthrop, H. Gollee, and I. D. Loram. 2013. Refractoriness in sustained visuo-manual control: Is the refractory duration intrinsic or does it depend on external system properties? PLoS Comput Biol 9, 1 (Jan. 2013), e1002843.

Herman Van Der Kooij and Erwin De Vlugt. 2007. Postural responses evoked by platform pertubations are dominated by continuous feedback. Journal of Neurophysiology 98, 2 (2007), 730-743.

Herman Van Der Kooij and Robert J. Peterka. 2011. Non-linear stimulus-response behavior of the human stance control system is predicted by optimization of a system with sensory and motor noise. fournal of Computational Neuroscience 30, 3 (2011), 759-778.

Eduardo Velloso, Marcus Carter, Joshua Newn, Augusto Esteves, Christopher Clarke, and Hans Gellersen. 2017. Motion correlation: Selecting objects by matching their movement. ACM Transactions on Computer-Human Interaction 24, 3 (2017), 1-35.

Qing Wang, Sanjeev R. Kulkarni, and Sergio Verdu. 2009. Divergence estimation for multidimensional densities via \$k\$Nearest-Neighbor distances. IEEE Transactions on Information Theory 55, 5 (May 2009), 2392-2405.

John Williamson and Roderick Murray-Smith. 2004. Pointing without a pointer. In Proceedings of the CHI'04 Extended Abstracts on Human Factors in Computing Systems. ACM, New York, NY, 1407-1410.

John Williamson, Roderick Murray-Smith, and Stephen Hughes. 2007. Shoogle: Excitatory multimodal interaction on mobile devices. In Proceedings of the SIGCHI Conference on Human Factors in Computing Systems. ACM, New York, NY, 121-124.

Jacob O. Wobbrock, Edward Cutrell, Susumu Harada, and I. Scott MacKenzie. 2008. An error model for pointing based on Fitts' law. In Proceedings of the SIGCHI Conference on Human Factors in Computing Systems. ACM, New York, NY, 1613-1622.

Daniel M. Wolpert and Zoubin Ghahramani. 2000. Computational principles of movement neuroscience. Nature Neuroscience 3, 11 (2000), 1212-1217.

Daniel M. Wolpert, R. Chris Miall, and Mitsuo Kawato. 1998. Internal models in the cerebellum. Trends in Cognitive Sciences 2, 9 (1998), 338-347.

Howard G. Wu, Yohsuke R. Miyamoto, Luis Nicolas Gonzalez Castro, Bence P. Ölveczky, and Maurice A. Smith. 2014. Temporal structure of motor variability is dynamically regulated and predicts motor learning ability. Nature Neuroscience 17, 2 (2014), 312.

Received June 2020; revised February 2021; accepted April 2021 